\title{
Lagrangian Rabinowitz Floer homology and twisted cotangent bundles
}

\section{Journal Article}

\section{Author(s):}

Merry, Will J.

Publication date:

2014-08

Permanent link:

https://doi.org/10.3929/ethz-b-000070886

Rights / license:

In Copyright - Non-Commercial Use Permitted

Originally published in:

Geometriae Dedicata 171(1), https://doi.org/10.1007/s10711-013-9903-9 


\title{
Lagrangian Rabinowitz Floer homology and twisted cotangent bundles
}

\author{
Will J. Merry
}

Received: 26 November 2011 / Accepted: 10 August 2013 / Published online: 1 September 2013

C) Springer Science+Business Media Dordrecht 2013

\begin{abstract}
We study the following rigidity problem in symplectic geometry: can one displace a Lagrangian submanifold from a hypersurface? We relate this to the Arnold Chord Conjecture, and introduce a refined question about the existence of relative leaf-wise intersection points, which are the Lagrangian-theoretic analogue of the notion of leaf-wise intersection points defined by Moser (Acta. Math. 141(1-2):17-34, 1978). Our tool is Lagrangian Rabinowitz Floer homology, which we define first for Liouville domains and exact Lagrangian submanifolds with Legendrian boundary. We then extend this to the 'virtually contact' setting. By means of an Abbondandolo-Schwarz short exact sequence we compute the Lagrangian Rabinowitz Floer homology of certain regular level sets of Tonelli Hamiltonians of sufficiently high energy in twisted cotangent bundles, where the Lagrangians are conormal bundles. We deduce that in this situation a generic Hamiltonian diffeomorphism has infinitely many relative leaf-wise intersection points.
\end{abstract}

Keywords Rabinowitz Floer homology · Leaf-wise intersections · Mañé critical value

Mathematics Subject Classification $53 \mathrm{D} 40 \cdot 57 \mathrm{R} 58 \cdot 37 \mathrm{~J} 45 \cdot 37 \mathrm{~J} 50$

\section{Introduction}

The aim of this paper is to study the following rigidity problem in symplectic geometry: can one displace a Lagrangian submanifold from a hypersurface? In order to fix the ideas, let us start with the following simple situation. Suppose $\left(X_{0}, \lambda_{0}\right)$ is Liouville domain, that is, $X_{0}$ is compact manifold with boundary $\Sigma:=\partial X_{0}$, and $d \lambda_{0}$ is a symplectic form on $X_{0}$ such that $\eta:=\left.\lambda_{0}\right|_{\Sigma}$ is a positive contact form on $\Sigma$. We attach to $X_{0}$ the positive part of the symplectization of $\Sigma$ to form $X:=X_{0} \cup_{\Sigma}(\Sigma \times[1, \infty))$. We extend $\lambda_{0}$ to a 1-form $\lambda$ defined on all of $X$ by setting $\lambda:=r \eta$ on $\Sigma \times\{r \geq 1\}$, and call $(X, \lambda)$ the completion

W. J. Merry $(\bowtie)$

Department of Mathematics, ETH Zürich, Switzerland

e-mail: merry@math.ethz.ch 
of the Liouville domain. Now suppose $L \subset X$ is an exact Lagrangian submanifold that is transverse to $\Sigma$ and is such that $K:=\Sigma \cap L$ is a closed Legendrian submanifold of $(\Sigma, \eta)$. Denote by $\theta^{t}: \Sigma \rightarrow \Sigma$ the Reeb flow of $\eta$. One can ask the following basic question.

Question 1.1 Is it possible to displace $\Sigma$ from L via a compactly supported Hamiltonian diffeomorphism?

This is related to the following better known question.

Question 1.2 Must there exist a Reeb chord with endpoints in $K$ ? That is, a point $p \in K$ such that $\theta^{\tau}(p) \in K$ for some $\tau \neq 0$.

The Arnold Chord Conjecture, which is still open, asserts a positive answer to Question 1.2 for any Legendrian $K$ in any contact manifold $(\Sigma, \eta)$. This conjecture was originally stated for Legendrian knots in $S^{3}$ (equipped with the standard contact structure) by Arnold in [8]. In dimension 3 the conjecture has been completely proved by Hutchings and Taubes [33,34]. In higher dimensions Mohnke [40] proved that the answer to Question 1.2 is 'yes' whenever the contact manifold arises as the boundary of a subcritical Stein manifold of odd dimension. There is also a Floer-theoretic proof that covers certain special cases of Mohnke's result which is due to Cieliebak [25]. Other results are due to Abbas [1], Ginzburg and Givental [31,32], and more recently, Bourgeois et al. [15] and Ritter [46].

Our first result is that a positive answer to Question 1.1 implies a positive answer to Question 1.2.

Theorem 1.3 Suppose $(X, \lambda)$ is a completion of a Liouville domain as above, and suppose that $L \subset X$ is an exact Lagrangian submanifold transverse to $\Sigma$ with the property that $K:=\Sigma \cap L$ is a closed Legendrian submanifold of $\left(\Sigma, \eta:=\left.\lambda\right|_{\Sigma}\right)$. If one can displace $\Sigma$ from $L$ via a compactly supported Hamiltonian diffeomorphism, then there exists a Reeb chord of $\eta$ with endpoints in $K$.

Remark 1.4 In fact, Theorem 1.3 can be deduced from Ritter's result [46] alluded to above. The main step in our proof Theorem 1.3 is to show that if the answer to Question 1.1 is 'yes' then the Lagrangian Rabinowitz Floer homology $\mathrm{RFH}_{*}(\Sigma, L, X)$ of $(\Sigma, L, X)$ vanishes. Then we observe that vanishing of the Lagrangian Rabinowitz Floer homology implies a positive answer to Question 1.2. In [46], Ritter proved that if the wrapped Floer homology $\mathrm{HW}_{*}(L)$ of $L$ vanishes then the answer to Question 1.2 is 'yes'. Current work in progress of Bounya [17] shows that $\mathrm{RFH}_{*}(\Sigma, L, X)=0$ if and only if $\mathrm{HW}_{*}(L)=0$. He proves this by constructing a short exact sequence relating the Lagrangian Rabinowitz Floer homology and the wrapped Floer homology, in a similar vein to Cieliebak, Frauenfelder and Oancea's result [21], which relates Rabinowitz Floer homology with symplectic homology. In this sense Theorem 1.2 covers exactly the same cases of the Chord Conjecture as Ritter's result.

Let us now discuss a refinement of Questions 1.1 and 1.2. Given a compactly supported Hamiltonian diffeomorphism $\psi: X \rightarrow X$, we say that a point $p \in K$ is a relative leaf-wise intersection point of $\psi$ if the orbit $\left\{\theta^{t}(p)\right\}_{t \in \mathbb{R}}$ intersects $\psi^{-1}(L)$. Equivalently, a point $p \in K$ is a relative leaf-wise intersection point if there exists $\tau \in \mathbb{R}$ such that

$$
\psi\left(\theta^{\tau}(p)\right) \in L .
$$

If for a given pair $\Sigma, L$ the answer to Question 1.1 is 'no', then it makes sense to ask the following question. 
Question 1.5 Suppose it is not possible to displace $\Sigma$ from L via a compactly supported Hamiltonian diffeomorphism. Is it then true that every $\psi$ has a relative leaf-wise intersection point?

Note a Reeb chord is just the special case $\psi=$ id. In both Question 1.2 and Question 1.5 one can also ask for multiplicity results. Many of the references given above (e.g. [25]) prove the existence of more than just one Reeb chord. We are interested in cases where there are infinitely many chords.

Question 1.6 When is it true that for a generic $\psi$ there always exist infinitely many relative leaf-wise intersection points?

It is possible to see relative leaf-wise intersection points for a given Hamiltonian diffeomorphism $\psi$ as critical points of a free-time Hamiltonian action functional $\mathscr{A}_{\psi}$ called the Rabinowitz action functional, that we define in Sect. 1.2. We construct a Floer theory for the functional $\mathscr{A}_{\psi}$, which we call the Lagrangian Rabinowitz Floer homology of $\mathscr{A}_{\psi}$. This is the Lagrangian intersection theoretic version of Rabinowitz. Floer homology, which was introduced by Cieliebak and Frauenfelder in [20], and used to detect (periodic) leaf-wise intersections by Albers and Frauenfelder in [3].

Remark 1.7 As mentioned above, there are many approaches to answering Question 1.2, including various Floer-theoretic ones. We believe that the main value of using Lagrangian Rabinowitz Floer homology in this setting is that it allows us attack the more refined Question 1.5. To the best of our knowledge, none of the previous methods can be directly used to answer Question 1.5. Another advantage is that Lagrangian Rabinowitz Floer homology can be defined in settings where symplectic/wrapped Floer homology cannot be. See Sect. 1.1 below.

In certain situations it is possible to compute the Lagrangian Rabinowitz Floer homology, which allows us to give an affirmative answer to Question 1.5 and establish a partial answer to Question 1.6. Here is one such setting: let $\left(M^{n}, g\right)$ denote a closed connected orientable Riemannian manifold of dimension $n \geq 2$, and consider the cotangent bundle $T^{*} M$ equipped with its canonical symplectic structure $d \lambda_{\text {can }}$, where $\lambda_{\text {can }}$ is the Liouville 1-form on $T^{*} M$. Recall that if $S^{d} \subset M$ is any closed connected submanifold, then the conormal bundle $N^{*} S$ is the submanifold of $T^{*} M$ given by

$$
N^{*} S:=\left\{(q, p) \in T^{*} M|q \in S, p|_{T_{q} S}=0\right\} .
$$

It is easy to see that $N^{*} S$ is a Lagrangian submanifold of $T^{*} M$. For instance, if $S=\{q\}$ is a point, then $N^{*} S=T_{q}^{*} M$, and if $S=M$, then $N^{*} S$ is the zero section $M \subset T^{*} M$. Denote by $P(M, S)$ the set of all smooth paths $q:[0,1] \rightarrow M$ with $q(0)$ and $q(1)$ both lying in $S$. We prove the following result, which is based on the work of Abbondandolo and Schwarz $[9,10]$.

Theorem 1.8 Let $\left(M^{n}, g\right)$ denote a closed connected orientable Riemannian manifold of dimension $n \geq 2$, and let $U^{*} M$ denote the unit cotangent bundle. Let $S^{d} \subseteq M$ denote a closed connected submanifold. Assume that one of the following two conditions hold:

1. $d<n / 2$, or $d=n / 2$ and $n \geq 4$,

2. The double coset space $\pi_{1}(S) \backslash \pi_{1}(M) / \pi_{1}(S)$ is non-trivial.

Then it is not possible to displace $U^{*} M$ from $N^{*} S$, and the answer to Question 1.5 is 'yes'. Moreover, if $\operatorname{dim} H_{*}\left(P(M, S) ; \mathbb{Z}_{2}\right)=\infty$ and the pair $\left(U^{*} M, N^{*} S\right)$ is non-degenerate (cf. Sect. 2.1-this condition is satisfied generically), then a generic Hamiltonian diffeomorphism has infinitely many relative leaf-wise intersection points.

Remark 1.9 The second condition in Theorem 1.8 is equivalent to the statement that $P(M, S)$ is not connected. 


\subsection{A more complicated setting}

In the main body of the paper we work in a somewhat more general setting than the one described above. This is due to our particular interest in twisted cotangent bundles. We introduce these shortly, but roughly speaking, the goal is to define Lagrangian Rabinowitz Floer homology and prove Theorem 1.3 in a sufficiently general setting that it applies to twisted cotangent bundles. We shall then compute the Lagrangian Rabinowitz Floer homology for certain hypersurfaces in twisted cotangent bundles, thus proving the analogue of Theorem 1.8. Unfortunately, this extra level of generality necessitates a less clean exposition. It is quite likely that some readers will only be interested in the setting described above. If this is the case, it is possible to skip large swathes of the paper, beginning with the rest of Sect. 1.1. We indicate in the exposition those sections that may safely be omitted.

We now define twisted cotangent bundles. As before, let $M$ denote a closed connected orientable $n$-dimensional manifold, where $n \geq 2$. Let $\pi: T^{*} M \rightarrow M$ denote the footpoint map $\pi(q, p) \mapsto q$, and let $\rho: \widetilde{M} \rightarrow M$ denote the universal cover of $M$. We write $\rho_{\sharp}: T^{*} \widetilde{M} \rightarrow T^{*} M$ for the map defined by $\rho_{\sharp}(p):=\left(D \rho(q)^{-1}\right)^{*}(p)$ for $p \in T_{\rho(q)}^{*} \widetilde{M}$. By convention, if $A \subseteq T^{*} M$ is any submanifold, we denote by $\widetilde{A}:=\rho_{\sharp}^{-1}(A)$. Note that $\widetilde{A}$ is in general not the universal cover of $A$ (see Remark 2.2). Suppose $\sigma \in \Omega^{2}(M)$ is a closed 2 -form. We pull $\sigma$ back to $T^{*} M$ and add it to the canonical symplectic form $d \lambda_{\text {can }}$ to obtain a new symplectic form

$$
\omega:=d \lambda_{\mathrm{can}}+\pi^{*} \sigma
$$

on $T^{*} M$. One calls $\omega$ a twisted symplectic form or a magnetic symplectic form. The latter terminology comes from viewing the Hamiltonian system on $\left(T^{*} M, \omega\right)$ defined by the Hamiltonian $(q, p) \mapsto \frac{1}{2}|p|^{2}$ as modeling the motion of a particle moving on $M$ under the effect of a magnetic field, represented by $\sigma$. We refer the reader to [5,30] for more information on twisted cotangent bundles. In this paper $\sigma$ may or may not be exact, but we always insist that $\sigma$ is weakly exact, that is, the lift $\widetilde{\sigma}:=\rho^{*} \sigma \in \Omega^{2}(\widetilde{M})$ is exact (which is equivalent to requiring that $\left.\sigma\right|_{\pi_{2}(M)}=0$ ). In fact, we will always make the additional assumption that $\widetilde{\sigma}$ admits a bounded primitive: there exists $\varphi \in \Omega^{1}(\widetilde{M})$ such that $d \varphi=\widetilde{\sigma}$ and such that

$$
\sup _{q \in \widetilde{M}}\left|\varphi_{q}\right|<\infty
$$

where the norm $|\cdot|$ is given by the lift of any Riemannian metric on $M$ to $\widetilde{M}$. Note that if $\sigma$ is not exact, then $\omega$ is also not exact. Thus twisted cotangent bundles do not fit into the setting discussed in the previous section. Following [22], we will develop the theory for hypersurfaces that are virtually contact, which is when the lifted hypersurface $\widetilde{\Sigma}:=\rho_{\sharp}^{-1}(\Sigma)$ is of contact type with respect to the lifted symplectic form $\widetilde{\omega}:=d \widetilde{\lambda}_{\text {can }}+\widetilde{\pi}^{*} \widetilde{\sigma}$ on $T^{*} \widetilde{M}$ (which is exact). Moreover the primitive $\lambda$ of $\widetilde{\omega}$ that restricts to define a contact form on $\widetilde{\Sigma}$ must be suitably bounded:

$$
\sup _{\widetilde{\Sigma}}|\lambda|<\infty, \quad \inf _{\widetilde{\Sigma}} \lambda(R)>0
$$

where $R$ is a vector field generating $\left.\operatorname{ker} \omega\right|_{\Sigma}$ pulled back to $T^{*} \widetilde{M}$. We work in the virtually contact setting because wide class of physically relevant hypersurfaces fall into this category: namely, all regular level sets of Tonelli Hamiltonians $H: T^{*} M \rightarrow \mathbb{R}$ for sufficiently high energy levels. Here we recall that the classical Tonelli assumption means that $H$ is fibrewise 
strictly convex and superlinear. In other words, the second differential $d^{2}\left(\left.H\right|_{T_{q}^{*} M}\right)$ of $H$ restricted to each tangent space $T_{q}^{*} M$ is positive definite, and

$$
\lim _{|p| \rightarrow \infty} \frac{H(q, p)}{|p|}=\infty
$$

uniformly for $q \in M$. As before, we are interested in applying the theory in the case where the Lagrangian is a conormal bundle $N^{*} S$. However since we are now working in a twisted cotangent bundle, not all conormal bundles are Lagrangian submanifolds. In fact it is not hard to see that $N^{*} S$ is a Lagrangian submanifold of $\left(T^{*} M, \omega\right)$ if and only if $\left.\sigma\right|_{S}=0$. If $\left.\sigma\right|_{S}=0$, then to any Tonelli Hamiltonian $H$ there is an associated Mañé critical value $c(H, \sigma, S) \in$ $\mathbb{R} \cup\{\infty\}$. The precise definition of the Mañé critical value $c(H, \sigma, S)$ is given in Sect. 3.1 below. Here we content ourselves with saying only that the dynamics of the Hamiltonian flow $\left.\phi_{H}^{t}\right|_{\Sigma}: \Sigma \cap N^{*} S \rightarrow \Sigma$ differ dramatically depending on whether $c(H, \sigma, S)$ is finite, and if it is, whether it is positive or negative. In this paper we are interested in the case where $c(H, \sigma, S)<0$, and hence we make the following definition.

Definition 1.10 Consider a closed connected hypersurface $\Sigma \subset T^{*} M$ and a closed connected submanifold $S$ such that $\left.\sigma\right|_{S}=0$, with $\Sigma \cap N^{*} S \neq \emptyset$ and $\Sigma \pitchfork N^{*} S$. The pair $(\Sigma, S)$ is called a Mañé supercritical pair if there exists a Tonelli Hamiltonian $H: T^{*} M \rightarrow \mathbb{R}$ with $c(H, \sigma, S)<0$, and such that $\Sigma$ is the regular level set $H^{-1}(0)$.

Lemma 3.4 below says that if $(\Sigma, S)$ is a Mañé supercritical pair then $\Sigma$ is of virtual restricted contact type. Let us now state versions of Theorem 1.3 and Theorem 1.8 that are valid in this more general setting-note that here a relative leaf-wise intersection point $p$ of a Hamiltonian diffeomorphism $\psi: X \rightarrow X$ is a point $p \in \Sigma \cap L$ such that the characteristic chord through $p$ intersects $\psi^{-1}(L)$. We refer the reader to Sect. 2 below for precise definitions of all the terms involved.

Theorem 1.11 Suppose $(X, \omega)$ is a geometrically bounded symplectically aspherical symplectic manifold with $c_{1}(T X)=0$. Let $\Sigma \subset X$ denote a closed connected $\pi_{1}$-injective hypersurface that encloses a compact connected component of $X \backslash \Sigma$, and let $L \subset X$ denote $a \pi_{1}$-injective Lagrangian submanifold transverse to $\Sigma$ with $\Sigma \cap L \neq \emptyset$. Let $\widetilde{X} \rightarrow X$ denote the universal cover of $X$. Assume there exists a primitive $\lambda$ of the lifted symplectic form $\widetilde{\omega}$ such that:

1. $\sup _{\widetilde{\Sigma}}|\lambda|<\infty$ and $\inf _{\widetilde{\Sigma}} \lambda(R)>0$, where $R$ is a vector field generating ker $\left.\omega\right|_{\Sigma}$ pulled back to $\widetilde{X}$.

2. $\left.\lambda\right|_{\widetilde{L}}=d$ (bounded function).

If there exists a compactly supported Hamiltonian diffeomorphism $\psi: X \rightarrow X$ with no relative leaf-wise intersection points (e.g. if one can displace $\Sigma$ from $L$ ), then there exists a characteristic chord of $\Sigma$ with endpoints in $\Sigma \cap L$.

Remark 1.12 The assumption that $c_{1}(T X)=0$ can be dropped, at the expense of losing the $\mathbb{Z}$-grading on the Lagrangian Rabinowitz Floer homology. The grading is inessential for Theorem 1.11. The construction is also perfectly valid if instead of a single Lagrangian submanifold $L$, we work with a pair $L_{0} \pitchfork L_{1}$ of $\pi_{1}$-injective Lagrangian submanifolds that intersect transversely over $\Sigma$. Additional assumptions would be needed for a $\mathbb{Z}$-grading on the Lagrangian Rabinowitz Floer homology, but again, this is not required for Theorem 1.11. In some sense the case $L_{0} \pitchfork L_{1}$ is easier, as then the Rabinowitz action functional can be assumed to be Morse, rather than Morse-Bott (cf. Lemma 2.13). Nevertheless, for the sake of a uniform presentation we work with one Lagrangian throughout the paper. 
As the discussion above indicates, the setup described in Theorem 1.11 is tailor-made to deal with twisted cotangent bundles. We next state a version of Theorem 1.8 valid for twisted cotangent bundles.

Theorem 1.13 Let $\left(M^{n}, g\right)$ denote a closed connected orientable Riemannian manifold of dimension $n \geq 2$, and let $\sigma \in \Omega^{2}(M)$ denote a weakly exact 2-form whose lift to the universal cover admits a bounded primitive. Equip $T^{*} M$ with the twisted symplectic form $\omega:=d \widetilde{\lambda}_{\text {can }}+\pi^{*} \sigma$. Let $S^{d} \subseteq M$ denote a closed connected submanifold such that $\left.\sigma\right|_{S}=0$, and let $\Sigma \subset T^{*} M$ denote a hypersurface such that $\left(\Sigma, N^{*} S\right)$ form a Mañé supercritical pair (cf. Definition 1.10). Assume that one of the following two conditions hold:

1. $d<n / 2$, or $d=n / 2$ and $n \geq 4$,

2. The double coset space $\pi_{1}(S) \backslash \pi_{1}(M) / \pi_{1}(S)$ is non-trivial.

Then it is not possible to displace $\Sigma$ from $N^{*} S$, and the answer to Question 1.5 is 'yes'. Moreover if $\operatorname{dim} H_{*}\left(P(M, S) ; \mathbb{Z}_{2}\right)=\infty$ and the pair $\left(\Sigma, N^{*} S\right)$ is non-degenerate (cf. Sect. 2.1-this condition is satisfied generically), then a generic Hamiltonian diffeomorphism has infinitely many relative leaf-wise intersection points.

\subsection{The method of proof}

We conclude the Introduction with a brief explanation of the proofs of Theorems 1.11 and 1.13. Actually, for simplicity here we restrict to the easier setting described at the beginning of the Introduction, where $(X, \lambda)$ is the completion of a Liouville domain and $L$ is an exact Lagrangian transversely intersecting $\Sigma=\partial X_{0}$ in a Legendrian submanifold $K$ (thus covering Theorems 1.3 and 1.8 instead of Theorems 1.11 and 1.13). Define $P(X, L)$ to be the set of smooth paths $x:[0,1] \rightarrow X$ satisfying $x(0), x(1) \in L$. Let $H: X \rightarrow \mathbb{R}$ denote a smooth function such that

$$
H(p, r)=h(r) \text { for }(p, r) \in \Sigma \times(0, \infty),
$$

where $h:(0, \infty) \rightarrow \mathbb{R}$ is a smooth increasing function that is constant on $\{r \leq 1 / 4\} \cup\{r \geq 3\}$ and equal to $r-1$ on $\left\{\frac{1}{2} \leq r \leq 2\right\}$. Suppose $\left.\lambda\right|_{L}=d l$. The Rabinowitz action functional

$$
\mathscr{A}: P(X, L) \times \mathbb{R} \rightarrow \mathbb{R}
$$

is defined by

$$
\mathscr{A}(x, \tau):=\int_{0}^{1} x^{*} \lambda+l(x(0))-l(x(1))-\tau \int_{0}^{1} H(x(t)) d t .
$$

The critical points of $\mathscr{A}$ come in two flavors: if $(x, \tau) \in \operatorname{Crit}(\mathscr{A})$ with $\tau \neq 0$, then the path $\zeta(t):=x(t / \tau)$ is a Reeb chord with endpoints in $L$. If $(x, 0) \in \operatorname{Crit}(\mathscr{A})$, then $x(t) \equiv p$ for some point $p \in K$. The Lagrangian Rabinowitz Floer homology $\operatorname{RFH}_{*}(\Sigma, L, X)$ is the homology of a chain complex generated by the critical points of $\mathscr{A}$. The boundary operator is defined by counting rigid solutions $u=(x, \tau): \mathbb{R} \rightarrow P(X, L) \times \mathbb{R}$ of the following pair of coupled second order non-linear elliptic partial differential equations:

$$
\left\{\begin{array}{l}
\partial_{s} x+J(x) \partial_{t} x=\tau \nabla H(x) \\
\partial_{s} \tau=\int_{0}^{1} H(x) d t
\end{array}\right.
$$

which connect different critical points of $\mathscr{A}$. Here we are simplifying the picture drastically, as critical points of $\mathscr{A}$ are never isolated. In reality we use Frauenfelder's theory [29] of 
gradient flow lines with cascades. As mentioned in Remark 1.4, the proof of Theorem 1.3 now uses the following two ingredients.

1. If there are no Reeb chords of $\left.\lambda\right|_{\Sigma}$ with endpoints in $K$, then one has $\operatorname{RFH}_{*}(\Sigma, L, X) \cong$ $\mathrm{H}_{*+(n-1) / 2}\left(K ; \mathbb{Z}_{2}\right)$. Indeed, in this case the critical point set of $\mathscr{A}$ can be identified with $K$ itself, and the boundary operator reduces to the boundary operator in Morse homology for a given Morse function on $K$ (this is because we count gradient flow lines with cascades; see Sect. 2.3).

2. If there exists a compactly supported Hamiltonian diffeomorphism with no relative leafwise intersection points, then $\operatorname{RFH}_{*}(\Sigma, L, X)=0$.

To motivate why (2) should be true, let us explain how the functional $\mathscr{A}$ can be tweaked to detect relative leaf-wise intersection points. This idea is due to Albers and Frauenfelder [3]. Suppose $\psi: X \rightarrow X$ is a compactly supported Hamiltonian diffeomorphism. Choose a compactly supported Hamiltonian $F_{t}: X \rightarrow \mathbb{R}$ such that $\psi=\phi_{F}^{1}$. Now define a new functional $\mathscr{A}_{\psi}$ by

$$
\mathscr{A}_{\psi}(x, \tau):=\int_{0}^{1} x^{*} \lambda+l(x(0))-l(x(1))-\eta \int_{0}^{1} \beta(t) H(x(t)) d t-\int_{0}^{1} \dot{\chi}(t) F_{\chi(t)}(x(t)) d t .
$$

Here $\beta: S^{1} \rightarrow \mathbb{R}$ is a smooth function with $\beta(t)=0$ for all $t \in\left[\frac{1}{2}, 1\right]$, and the integral $\int_{0}^{1} \beta(t) d t$ is equal to 1 , and $\chi:[0,1] \rightarrow[0,1]$ is a smooth monotone map with $\chi\left(\frac{1}{2}\right)=0$ and $\chi(1)=1$. The point of the two cutoff functions $\beta$ and $\chi$ is to ensure that $\beta(t) H(x)$ and $\dot{\chi}(t) F_{\chi(t)}(x)$ have disjoint time support. This implies that if $(x, \tau) \in \operatorname{Crit}\left(\mathscr{A}_{\psi}\right)$, then $x(0) \in K$, and for $t \in\left[0, \frac{1}{2}\right]$ one has $x(t)=\theta^{\beta(t)}(x(0))$, and for $t \in\left[\frac{1}{2}, 1\right]$ one has $x(t)=\phi_{F}^{\chi(t)}\left(x\left(\frac{1}{2}\right)\right)$. In other words, if $p:=x(0)$, then $p \in K$ and $\psi\left(\theta^{\tau}(p)\right) \in L$. Thus $p$ is a relative leaf-wise intersection point of $\psi$. The key point now is that one can define the Rabinowitz Floer homology $\operatorname{RFH}_{*}\left(\mathscr{A}_{\psi}\right)$ for $\mathscr{A}_{\psi}$ as well, and in fact the Rabinowitz Floer homology is unchanged:

$$
\operatorname{RFH}_{*}\left(\mathscr{A}_{\psi}\right) \cong \operatorname{RFH}_{*}(\Sigma, L, X) .
$$

This should be viewed in the same spirit as the fact that the Morse [resp. Floer] homology of a closed [symplectic] manifold is independent of the Morse [resp. Hamiltonian] function. Now statement (2) above is clear: if $\psi$ has no relative leaf-wise intersection points, then the corresponding functional $\mathscr{A}_{\psi}$ has no critical points-and thus $\operatorname{RFH}\left(\mathscr{A}_{\psi}\right)=0$. It remains to explain how the computation of $\mathrm{RFH}_{*}\left(\Sigma, N^{*} S, T^{*} M\right)$ is made. We extend to the Lagrangian setting the Abbondandolo and Schwarz [10] short exact sequence, which relates the Lagrangian Rabinowitz Floer chain complex to the Morse complex of an appropriate free time action functional. The homology of this complex is (roughly speaking) the singular homology of the space $P(M, S)$. In our earlier paper [37] we extended the short exact sequence from [10] to the setting of twisted cotangent bundles, and the idea here is very similar.

\section{Preliminaries}

Here are some notational conventions.

- We denote by $C_{\mathrm{ct}}^{\infty}(X, \mathbb{R})$ the set of functions on $X$ which are constant outside of a compact set, and by $C_{0}^{\infty}(X, \mathbb{R}) \subset C_{\mathrm{ct}}^{\infty}(X, \mathbb{R})$ the subset of compactly supported functions. 
- We use the (non-standard) sign convention that an almost complex structure $J$ on a symplectic manifold $(X, \omega)$ is $\omega$-compatible if $g_{J}:=\omega(J \cdot, \cdot)$ is a Riemannian metric on $X$. We denote by $\mathscr{J}(X, \omega)$ the set of all $\omega$-compatible almost complex structures on $X$.

- Given a family $\mathbf{J}=\left(J_{t}\right)_{t \in[0,1]} \subset \mathscr{J}(X, \omega)$, and $(x, \tau) \in C^{\infty}([0,1], X) \times \mathbb{R}$, we use the special notation $\langle\langle\cdot, \cdot\rangle\rangle_{\mathbf{J}}$ to denote the inner product on $C^{\infty}\left(x^{*} T X\right) \times \mathbb{R}$ defined by

$$
\left\langle\left\langle(\xi, h),\left(\xi^{\prime}, h^{\prime}\right)\right)\right\rangle_{\mathbf{J}}:=\int_{0}^{1} g_{J_{t}}\left(\xi(t), \xi^{\prime}(t)\right) d t+h h^{\prime} .
$$

- The symplectic gradient $X_{H} \in \operatorname{Vect}(X)$ of a Hamiltonian $H: X \rightarrow \mathbb{R}$ is defined by $i_{X_{H}} \omega=-d H$. Thus the gradient $\nabla H$ of $H$ with respect to $g_{J}$ is given by $\nabla H=J X_{H}$.

In this section we introduce the precise setting in which we define the Lagrangian Rabinowitz Floer homology. We are aiming for Definition 2.7, which introduces the notion of a Rabinowitz admissible triple $(\Sigma, L, \alpha)$. This is the setting that we will prove Theorem $1.11 \mathrm{in}$.

Remark 2.1 If however the reader is only interested in the setting described at the beginning of the Introduction, things become much simpler, and the reader may skim this entire section apart from Remark 2.8, where we explicitly consider this special case.

Let $\left(X^{2 n}, \omega\right)$ denote a connected non-compact symplectic manifold such that:

1. $(X, \omega)$ is geometrically bounded - this means that there exist $\omega$-compatible almost complex structures $J$ on with the property that the Riemannian metric $g_{J}(\cdot, \cdot):=\omega(J \cdot, \cdot)$ is complete, has bounded sectional curvature and has injectivity radius bounded away from zero

2. The first Chern class $c_{1}(T X, J)$ is zero (for some, and hence any $J \in \mathscr{J}(X, \omega)$ ).

3. The symplectic form $\omega$ is symplectically aspherical. This means that for every smooth map $f: S^{2} \rightarrow X$, one has $\int_{S^{2}} f^{*} \omega=0$.

Assumption (2) is made for simplicity only, and could be weakened at the expense of losing the $\mathbb{Z}$-grading on the Lagrangian Rabinowitz Floer homology. Assumption (1) however is much more crucial, and cannot be dispensed with. If we denote by $\rho: \widetilde{X} \rightarrow X$ the universal cover of $X$ and by $\widetilde{\omega}:=\rho^{*} \omega \in \Omega^{2}(\widetilde{X})$ then Assumption (3) is equivalent to requiring that $\widetilde{\omega}$ is exact. Our main interest in such symplectic manifolds is due to the fact that the twisted cotangent bundles introduced in Sect. 1.1 satisfy these requirements-see Sect. 3.1.

Remark 2.2 Suppose $A \subseteq X$ is a submanifold. We denote by $A^{\text {univ }} \rightarrow A$ the universal cover of $A$, and by $\widetilde{A}:=\rho^{-1}(A) \subseteq \widetilde{X}$. Note in general, $A^{\text {univ }} \neq \widetilde{A}$ if $A \varsubsetneqq X$. We say that $A$ is $\pi_{1}$-injective if the inclusion $A \hookrightarrow X$ induces an injection $\pi_{1}(A) \rightarrow \pi_{1}(X)$. In this case $\widetilde{A}$ is a disjoint union of components each diffeomorphic to $A^{\text {univ }}$. In particular, each component of $\widetilde{A}$ is simply connected, and thus $\mathrm{H}^{1}(\widetilde{A} ; \mathbb{Z})=0$.

Recall that for a closed connected orientable hypersurface $\Sigma \subset X$, there is a distinguished oriented line bundle ker $\omega \rightarrow \Sigma$ over $\Sigma$ called the characteristic line bundle. We denote by $\mathscr{D}(\Sigma)$ the set of Hamiltonians $H \in C^{\infty}(X, \mathbb{R})$ with the property that $\Sigma$ is the regular energy level $H^{-1}(0)$, and that the symplectic gradient $\left.X_{H}\right|_{\Sigma}$ is a positively oriented section of ker $\omega$, and we write $\mathscr{D}_{\mathrm{ct}}(\Sigma):=\mathscr{D}(\Sigma) \cap C_{\mathrm{ct}}^{\infty}(X, \mathbb{R})$. A characteristic chord of $\Sigma$ with endpoints in some specified Lagrangian submanifold $L$ of $X$ is a flow line of $\phi_{H}^{t}$ for some $H \in \mathscr{D}(\Sigma)$ which starts and ends in $\Sigma \cap L$. This is independent of the choice of $H \in \mathscr{D}(\Sigma)$ since for $H_{1}, H_{2} \in \mathscr{D}(\Sigma)$ the flows $\left.\phi_{H_{1}}^{t}\right|_{\Sigma}$ and $\left.\phi_{H_{2}}^{t}\right|_{\Sigma}$ differ only by a time change. We are primarily interested in the case when the hypersurface $\Sigma$ satisfies the following condition, which was introduced by Cieliebak et al. [22]. 
Definition 2.3 A closed connected hypersurface $\Sigma$ is of virtual restricted contact type if (a) $\Sigma$ is $\pi_{1}$-injective, (b) $\Sigma$ encloses a compact connected component of $X \backslash \Sigma$ and (c), there exists a primitive $\lambda$ of $\widetilde{\omega}$ such that:

1. For some (and hence any) Riemannian metric $g$ on $\Sigma$, there exists a constant $C=C(g)<$ $\infty$ such that

$$
\sup _{x \in \widetilde{\Sigma}}\left|\lambda_{x}\right| \leq C,
$$

where $|\cdot|$ denotes the lift of $g$ to $\widetilde{\Sigma}$.

2. For some (and hence any) non-vanishing positively oriented section $R$ of ker $\omega$, there exists a constant $\varepsilon=\varepsilon(R)>0$ such that

$$
\inf _{x \in \widetilde{\Sigma}} \lambda(\widetilde{R}(x)) \geq \varepsilon
$$

where $\widetilde{R}$ denotes a lift of $R$ to $\widetilde{X}$.

We now discuss the Lagrangians that we consider. All Lagrangian submanifolds in this paper are assumed to be connected, even if this is not explicitly stated. Suppose we are given a Lagrangian submanifold $L$ of $X$ that is $\pi_{1}$-injective. Since we assume that $\omega$ is symplectically aspherical and $c_{1}(T X)=0$, the $\pi_{1}$-injectivity assumption implies that $\left.\omega\right|_{\pi_{2}(X, L)}=\left.c_{1}\right|_{\pi_{2}(X, L)}=0$. Since $^{1}(\widetilde{L} ; \mathbb{Z})=0\left(\right.$ cf. Remark 2.2) and $\left.\omega\right|_{L}=0$, if $\lambda \in \Omega^{1}(\tilde{X})$ is a primitive of $\widetilde{\omega}$, we can find a smooth function $l: \widetilde{L} \rightarrow \mathbb{R}$ such that $\left.\lambda\right|_{\widetilde{L}}=d l$.

Definition 2.4 We say that a $\pi_{1}$-injective Lagrangian $L$ is virtually exact if one can choose a primitive $\lambda$ of $\widetilde{\omega}$ and a function $l$ such that $\left.\lambda\right|_{\tilde{L}}=d l$, where $l \in C^{\infty}(\widetilde{L}, \mathbb{R})$ is a bounded function.

Now we explain the notion of a good primitive of $\widetilde{\omega}$.

Definition 2.5 Suppose we have fixed a pair $\Sigma, L$ consisting of a hypersurface $\Sigma$ of virtual restricted contact type and a virtually exact Lagrangian. A primitive $\lambda$ of $\widetilde{\omega}$ is called good with respect to $\Sigma, L$ (or just good if $\Sigma$ and $L$ are understood) if $\lambda$ satisfies (2.2) and (2.3), and has the property that $\left.\lambda\right|_{\widetilde{L}}=d$ (bounded function).

Next, we define the appropriate notion of homotopy:

Definition 2.6 Suppose that $\Sigma$ is a hypersurface of virtual restricted contact type, $L$ is a virtually exact Lagrangian, and $\lambda$ is a good primitive for $(\Sigma, L)$. Fix $H \in \mathscr{D}_{\mathrm{ct}}(\Sigma)$. A good homotopy is a family $\left(H_{s}, \lambda_{s}\right)_{s \in(-\varepsilon, \varepsilon)}$ such that:

1. $\left(H_{S}\right)$ is a smooth family of uniformly compactly supported Hamiltonians such that $H_{0}=$ $H$, and such that $\Sigma_{s}:=H_{s}^{-1}(0)$ is of virtual restricted contact type for each $s \in(-\varepsilon, \varepsilon)$, with $H_{s} \in \mathscr{D}_{\mathrm{ct}}\left(\Sigma_{s}\right)$;

2. $\left(\lambda_{s}\right)$ is a smooth family of 1 -forms such that $\lambda_{0}=\lambda$ and $\lambda_{s}$ is a good primitive with respect to $\Sigma_{s}, L$, and such that the constants in (2.2) and (2.3) may be taken independently of $s$, and if $\left.\lambda_{s}\right|_{\tilde{L}}=d l_{s}$ then $\sup _{s}\left\|l_{s}\right\|<\infty$.

Let us fix once and for all a point $\star \in X$. When talking about Lagrangian submanifolds $L$ of $X$, we shall always implicitly assume that $\star \in L$. Let $P(X, L)$ denote the set of smooth maps $x:[0,1] \rightarrow X$ with $x(0) \in L$ and $x(1) \in L$. Define

$$
\Pi_{L}:=\pi_{1}(X, \star) / \sim,
$$


where for $\mathrm{a}, \mathrm{b} \in \pi_{1}(\mathrm{X}, \star)$ we have $\mathrm{a} \sim \mathrm{b}$ if and only if there exists $\mathrm{C}_{0}, \mathrm{C}_{1} \in \pi_{1}(\mathrm{~L}, \star)$ such that

$$
\mathrm{a}=\mathrm{c}_{0} \mathrm{bc}_{1}
$$

(i.e. $\Pi_{L}$ is the double coset space $\left.\pi_{1}(L, \star) \backslash \pi_{1}(X, \star) / \pi_{1}(L, \star)\right)$. It is not hard to see that $\Pi_{L} \cong \pi_{0}\left(P(X, L)\right.$ ) (see for instance [45, Lemma 3.3.1]). Given $\alpha \in \Pi_{L}$, we denote by $P_{\alpha}(X, L)$ the connected component of $P(X, L)$ corresponding to $\alpha$, so that

$$
P(X, L)=\bigsqcup_{\alpha \in \Pi_{L}} P_{\alpha}(X, L) .
$$

Let us now fix for each $\alpha \in \Pi_{L}$ a smooth loop $x_{\alpha}: S^{1} \rightarrow X$ with $x_{\alpha}(0)=\star$ such that $x_{\alpha}$ represents $\alpha$. It is convenient to choose these loops $x_{\alpha}$ so that the class $0 \in \Pi_{L}$ is represented by the constant path $x_{0}(t) \equiv \star$, and such that $x_{\alpha}(t)=x_{-\alpha}(1-t)$. Fix a point $\widetilde{\star} \in \widetilde{X}$ that projects onto $\star$, and for each $\alpha \in \Pi_{L}$ let $\widetilde{x}_{\alpha}:[0,1] \rightarrow \widetilde{X}$ denote the unique lift of $x_{\alpha}$ with $\widetilde{x}_{\alpha}(0)=\widetilde{\star}$. In particular, $\widetilde{x}_{0}(t)=\widetilde{\star}$ for all $t$. Given $x \in P_{\alpha}(X, L)$, let us say that a map $\bar{x}:[0,1] \times[0,1] \rightarrow X$ is a filling of $x$ if $\bar{x}$ satisfies:

- $\bar{x}(0, t)=x(t)$,

- $\bar{x}(1, t)=x_{\alpha}(t)$, and

- $\bar{x}([0,1] \times\{0,1\}) \subset L$.

If $f: S^{1} \rightarrow P(X, L)$ is a smooth loop then we may alternatively think of $f$ as a map $f: S^{1} \times[0,1] \rightarrow X$ with $f\left(S^{1} \times\{0,1\}\right) \subset L$. We will only work with classes $\alpha \in \Pi_{L}$ for which the following condition is satisfied:

(A) If $f: S^{1} \rightarrow P_{\alpha}(X, L)$ is any smooth loop then $\int_{S^{1} \times[0,1]} f^{*} \omega=0$.

Since $L$ is $\pi_{1}$-injective and $\left.\omega\right|_{\pi_{2}(X)}=0$, one has $\left.\omega\right|_{\pi_{2}(X, L)}=0$, and thus (A) is satisfied for the element $0 \in \Pi_{L}$. When (A) is satisfied we can define the symplectic area functional $\Omega: P_{\alpha}(X, L) \rightarrow \mathbb{R}$ by

$$
\Omega(x):=\int_{[0,1] \times[0,1]} \bar{x}^{*} \omega,
$$

where $\bar{x}$ is any filling of $x$ (that is, (A) implies that $\Omega$ is well defined). The precise conditions under which we will define the Lagrangian Rabinowitz Floer homology is given by the following definition.

Definition 2.7 A triple $(\Sigma, L, \alpha)$ is called Rabinowitz admissible if:

- $\Sigma$ is a hypersurface of virtual restricted contact type,

- $L$ is a virtually exact Lagrangian submanifold,

- $\Sigma \pitchfork L, \Sigma \cap L \neq \emptyset$,

- $\alpha \in \Pi_{L}$ satisfies (A),

- There exist good primitives $\lambda$ of $\widetilde{\omega}$.

Remark 2.8 As promised at the beginning of this section (see Remark 2.1), we summarize in this Remark the simplifications that one can make to Definition 2.7 if one works in the setting described at the beginning of the Introduction. Recall here we take $X$ to be the completion of a Liouville domain. Here one starts with a a compact manifold $X_{0}$ with boundary $\Sigma:=\partial X_{0}$, equipped with an exact symplectic form $d \lambda_{0}$ such that $\eta:=\left.\lambda_{0}\right|_{\Sigma}$ is a positive contact form on $\Sigma$. We attach to $X_{0}$ the positive part of the symplectization of $\Sigma$ to 
form $X:=X_{0} \cup_{\Sigma}(\Sigma \times[1, \infty))$. We extend $\lambda_{0}$ to a 1-form $\lambda$ defined on all of $X$ by setting $\lambda:=r \eta$ on $\Sigma \times\{r \geq 1\}$. Suppose $L \subset X$ is an exact Lagrangian submanifold which is transverse to $\Sigma$ and is such that $K:=\Sigma \cap L$ is a closed Legendrian submanifold of $(\Sigma, \eta)$. In addition we make the assumption that

$$
L \cap(\Sigma \times\{r \geq 1\})=K \times\{r \geq 1\} .
$$

This condition (2.6) implies that one can write $\left.\lambda\right|_{L}=d l$ for some function $l: L \rightarrow \mathbb{R}$ that vanishes to infinite order along $K$, and is the analogue in this setting to asking that good primitives in the sense of Definition 2.5 exist. If $L$ does not satisfy (2.6) then one can deform $L \cap X_{0}$ via a Hamiltonian isotopy of $X_{0}$ relative to $\Sigma$ to obtain a new Lagrangian submanifold $L_{0}^{\prime}$ of $X_{0}$ with the property that if $L^{\prime}:=L_{0}^{\prime} \cup_{K}(K \times[1, \infty))$ then $L^{\prime}$ satisfies (2.6) (see [13, Lemma 3.1]). Since in this case the symplectic form on $X$ is exact, every class $\alpha \in \Pi_{L}$ satisfies the condition (A) introduced on the preceding page. In this case we define the symplectic area functional $\Omega: P(X, L) \rightarrow \mathbb{R}$ by

$$
\Omega(x):=\int_{0}^{1} x^{*} \lambda+l(x(0))-l(x(1)) .
$$

2.1 The Rabinowitz action functional

We now introduce the Rabinowitz action functional. To begin with let us assume $L \subset X$ is a virtually exact Lagrangian and $\alpha \in \Pi_{L}$ is a class satisfying the condition (A) defined on the previous page. Thus the symplectic area functional $\Omega: P_{\alpha}(X, L) \rightarrow \mathbb{R}$ from (2.5) is well defined.

Definition 2.9 Let $H \in C_{\mathrm{ct}}^{\infty}(X, \mathbb{R})$ and assume that 0 is a regular value of $H$, with $H^{-1}(0) \cap$ $L \neq \varnothing$ and $H^{-1}(0) \pitchfork L$. The Rabinowitz action functional $\mathscr{A}_{H}: P_{\alpha}(X, L) \times \mathbb{R} \rightarrow \mathbb{R}$ is defined by

$$
\mathscr{A}_{H}(x, \tau)=\Omega(x)-\tau \int_{0}^{1} H(x) d t .
$$

An easy computation shows that the critical points of $\mathscr{A}_{H}$ are pairs $(x, \tau)$ such that

$$
\begin{aligned}
& \dot{x}=\tau X_{H}(x(t)) \text { for all } t \in[0,1], \\
& \int_{0}^{1} H(x) d t=0 .
\end{aligned}
$$

Since $H$ is invariant under its Hamiltonian flow, the second equation implies

$$
H(x(t))=0 \text { for all } t \in[0,1],
$$

and so

$$
x([0,1]) \subset H^{-1}(0) .
$$

Thus if we denote by $\mathrm{Crit}^{\alpha}\left(\mathscr{A}_{H}\right)$ the set of critical points of $\mathscr{A}_{H}$ then $(x, \tau)$ belongs to $\mathrm{Crit}^{\alpha}\left(\mathscr{A}_{H}\right)$ if and only if

$$
\dot{x}=\tau X_{H}(x), \quad x([0,1]) \subset H^{-1}(0) .
$$


If $(x, \tau) \in \operatorname{Crit}^{\alpha}\left(\mathscr{A}_{H}\right)$ with $\tau \neq 0$ then $\zeta(t):=x(t / \tau)$ is a flow line of $\phi_{H}^{t}$. If $\alpha \neq 0$ these are the only possible critical points. However if $\alpha=0$ and $p \in H^{-1}(0) \cap L$ then $(p, 0) \in \mathrm{Crit}^{0}\left(\mathscr{A}_{H}\right)$, where $p$ is also thought of as the constant path $t \mapsto p$. Note that if $(x, \tau) \in \mathrm{Crit}^{\alpha}\left(\mathscr{A}_{H}\right)$ then

$$
\mathscr{A}_{H}(x, \tau)=\Omega(x) .
$$

Given $-\infty<a<b<\infty$, denote by

$$
\mathrm{Crit}^{\alpha}\left(\mathscr{A}_{H}\right)_{a}^{b}:=\left\{(x, \tau) \in \mathrm{Crit}^{\alpha}\left(\mathscr{A}_{H}\right) \mid a \leq \mathscr{A}_{H}(x, \tau) \leq b\right\} .
$$

We always implicitly assume when referring to action windows that the endpoints $a$ and $b$ are not critical values of $\mathscr{A}_{H}$. Suppose $\mathbf{J}=\left(J_{t}\right)_{t \in[0,1]} \subset \mathscr{J}(X, \omega)$. We let $\nabla_{\mathbf{J}} \mathscr{A}_{H}$ denote the gradient of $\mathscr{A}_{H}$ with respect to $\langle\langle\cdot, \cdot\rangle\rangle_{\mathbf{J}}$ (cf. (2.1)), so that

$$
\nabla_{\mathbf{J}} \mathscr{A}_{H}(x, \tau)=\left(\begin{array}{c}
J_{t}(x)\left(\dot{x}-\tau X_{H}(x)\right) \\
-\int_{0}^{1} H(x) d t
\end{array}\right) .
$$

Given $(x, \tau) \in \operatorname{Crit}\left(\mathscr{A}_{H}\right)$ let us denote by

$$
\nabla_{\mathbf{J}}^{2} \mathscr{A}_{H}(x, \tau): W^{1, r}\left(x^{*} T X\right) \oplus \mathbb{R} \rightarrow L^{r}\left(x^{*} T X\right) \oplus \mathbb{R}
$$

(for some fixed $r \geq 2$ ) the operator obtained by linearizing $\nabla_{\mathbf{J}} \mathscr{A}_{H}(x, \tau)$, that is,

$$
\nabla_{\mathbf{J}}^{2} \mathscr{A}_{H}(x, \tau)(\xi, h):=\left.\frac{\partial}{\partial s}\right|_{s=0} \nabla_{\mathbf{J}} \mathscr{A}_{H}\left(x_{s}, \tau_{s}\right),
$$

where $\left(x_{s}, \tau_{s}\right)_{s \in(-\varepsilon, \varepsilon)} \subset P_{\alpha}(X, L) \times \mathbb{R}$ satisfies

$$
\left.\frac{\partial}{\partial s}\right|_{s=0}\left(x_{s}, \tau_{s}\right)=(\xi, h) .
$$

One computes that

$$
\nabla_{\mathbf{J}}^{2} \mathscr{A}_{H}(x, \tau)\left(\begin{array}{l}
\xi \\
h
\end{array}\right)=\left(\begin{array}{c}
J_{t}(x) \nabla_{t} \xi+\left(\nabla_{\xi} J_{t}\right) \dot{x}-\tau \nabla_{\xi} \nabla_{J_{t}} H-h \nabla_{J_{t}} H \\
-\int_{0}^{1} d H(\xi) d t
\end{array}\right),
$$

where $\nabla_{J_{t}} H$ denotes the gradient of $H$ with respect to the metric $g_{J_{t}}$.

Definition 2.10 A gradient flow line of $(H, \mathbf{J})$ is a smooth map $u=(x, \tau): \mathbb{R} \rightarrow$ $P_{\alpha}(X, L) \times \mathbb{R}$ such that

$$
\partial_{s} u+\nabla_{\mathbf{J}} \mathscr{A}_{H}(u(s))=0 .
$$

In components this reads:

$$
\begin{aligned}
& \partial_{s} x+J_{t}(x)\left(\partial_{t} x-\tau X_{H}(x)\right)=0 ; \\
& \partial_{s} \tau-\int_{0}^{1} H(x) d t=0 .
\end{aligned}
$$

Given $-\infty<a<b<\infty$, denote by $\mathscr{M}^{\alpha}(H, \mathbf{J})_{a}^{b}$ the set of gradient flow lines $u$ of $(H, \mathbf{J})$ that satisfy $a \leq \mathscr{A}_{H}(u(s)) \leq b$ for all $s \in \mathbb{R}$. 
It will often be useful to let both $H$ and $\mathbf{J}$ depend additionally on a parameter $s \in \mathbb{R}$. Suppose $\left(H_{S}\right)_{s \in \mathbb{R}} \subset C_{\mathrm{ct}}^{\infty}(X, \mathbb{R})$ is a smooth family of Hamiltonians, which is asymptotically constant in the sense that there exist $H_{ \pm} \in C_{\mathrm{ct}}^{\infty}(X, \mathbb{R})$ such that $H_{s}=H_{-}$for $s \ll 0$ and $H_{s}=H_{+}$for $s \gg 0$. Assume that 0 is a regular value of both $H_{-}$and $H_{+}$, with $H_{ \pm}(0) \cap L \neq \varnothing$ and $H_{ \pm}^{-1}(0) \pitchfork L$. Similarly, suppose we are given a family $\left(\mathbf{J}_{s}=\left(J_{s, t}\right)\right)_{s \in \mathbb{R}} \subset \mathscr{J}(X, \omega)$ of almost complex structures which is also asymptotically constant in the sense above. One can then study the $s$-dependent equation

$$
\partial_{s} u+\nabla_{\mathbf{J}_{s}} \mathscr{A}_{H_{s}}(u(s))=0,
$$

and given $-\infty<a<b<\infty$, we denote by $\mathscr{M}^{\alpha}\left(H_{s}, \mathbf{J}_{s}\right)_{a}^{b}$ the set of smooth maps $u=(x, \tau)$ that satisfy this equation together with the asymptotic conditions

$$
\lim _{s \rightarrow-\infty} \mathscr{A}_{H_{s}}(u(s)) \leq b, \quad \lim _{s \rightarrow \infty} \mathscr{A}_{H_{s}}(u(s)) \geq a .
$$

Note that if $\left(H_{s}, \mathbf{J}_{s}\right)=(H, \mathbf{J})$ does not depend on $s$ then $\mathscr{M}^{\alpha}\left(H_{s}, \mathbf{J}_{s}\right)_{a}^{b}=\mathscr{M}^{\alpha}(H, \mathbf{J})_{a}^{b}$. Given a gradient flow line $u$, we denote by

$$
D_{u}: W^{1, r}\left(x^{*} T X\right) \oplus W^{1, r}(\mathbb{R}, \mathbb{R}) \rightarrow L^{r}\left(x^{*} T X\right) \oplus L^{r}(\mathbb{R}, \mathbb{R})
$$

the linear operator given by

$$
D_{u}\left(\begin{array}{l}
\xi \\
h
\end{array}\right):=\left(\begin{array}{c}
\nabla_{s} \xi+J_{t}(x) \nabla_{t} \xi+\left(\nabla_{\xi} J_{t}\right) \partial_{t} x-\tau \nabla_{\xi} \nabla_{J_{t}} H-h \nabla_{J_{t}} H \\
\partial_{s} h-\int_{0}^{1} d H(x)(\xi) d t
\end{array}\right) .
$$

Note that in the special case where $u(s)=(x, \tau) \in \mathrm{Crit}^{\alpha}\left(\mathscr{A}_{H}\right)$ we have $D_{u}=\nabla_{\mathbf{J}}^{2} \mathscr{A}_{H}(x, \tau)$. Suppose $(x, \tau) \in \mathrm{Crit}^{\alpha}\left(\mathscr{A}_{H}\right)$ with $\tau \neq 0$. The nullity of $(x, \tau)$ is the integer

$$
n(x, \tau):=\operatorname{dim} D \phi_{H}^{\tau}(x(0))\left(T_{x(0)} L\right) \cap T_{x(1)} L .
$$

We say that $(x, \tau)$ is non-degenerate if $n(x, \tau)=0$. It is well known that this implies that $\nabla_{\mathbf{J}}^{2} \mathscr{A}_{H}(x, \tau)$ is bijective. We wish to associate an integer $\chi(x, \tau) \in\{-1,1\}$ to each non-degenerate critical point $(x, \tau)$ with $\tau \neq 0$. As proved by Albers and Frauenfelder [2, Proposition B.1], if $(x, \tau)$ is non-degenerate then we can find a smooth family $\bar{\zeta}_{s} \in P(X, L)$ and a smooth function $s \mapsto \tau(s)$ such that $\tau(0)=|\tau|$ and $\bar{\zeta}_{0}=x$. We set

$$
\zeta_{s}(t):=\bar{\zeta}_{s}(t / \tau(s))
$$

Moreover one also has $\tau^{\prime}(0) \neq 0$, and the function

$$
e(s):=H\left(\zeta_{s}(0)\right)
$$

is smooth. Note that $e(0)=0$. In fact, one has $e^{\prime}(0) \neq 0$. Since this last statement is not proved in [2, Proposition B.1], let us quickly show this: define $\xi \in C^{\infty}\left(x^{*} T X\right)$ by $\xi(t):=\left.\frac{\partial}{\partial s}\right|_{s=0} \zeta_{s}(t \tau(s))$. Then a direct computation shows that

$$
\nabla_{\mathbf{J}}^{2} \mathscr{A}_{H}(x, \tau)\left(\xi, \tau^{\prime}(0)\right)=\left(0, e^{\prime}(0)\right)
$$

and thus as $\nabla_{\mathbf{J}}^{2} \mathscr{A}_{H}(x, \tau)$ is bijective and $\tau^{\prime}(0) \neq 0$ we must have $e^{\prime}(0) \neq 0$. Anyway, the upshot is that it makes sense to define the correction term

$$
\chi(x, \tau):=\operatorname{sign}(\tau) \cdot \operatorname{sign}\left(-\frac{e^{\prime}(0)}{\tau^{\prime}(0)}\right) .
$$


Remark 2.11 A priori, it would appear that the correction term $\chi(x, \tau)$ could depend on the choice of family $\left(\zeta_{s}\right)$. In fact, this is not the case, as is proved in [38, Lemma 5.12].

Remark 2.12 In the simpler setting described in Remark 2.8 one can show that $\chi(x, \tau)=$ $\operatorname{sign}(\tau)$. See [38, Section 2.3].

If $p \in H^{-1}(0) \cap L$ then $(p, 0) \in \operatorname{Crit}^{0}\left(\mathscr{A}_{H}\right)$. Since we are assuming that $H^{-1}(0) \pitchfork L$, it is reasonable to call all these critical points non-degenerate as well. The next Lemma motivates this.

Lemma 2.13 If $\alpha \neq 0$ and all critical points of $\mathscr{A}_{H}$ belonging to $P_{\alpha}(X, L) \times \mathbb{R}$ are nondegenerate then $\mathscr{A}_{H}: P_{\alpha}(X, L) \times \mathbb{R} \rightarrow \mathbb{R}$ is a Morse functional and Crit ${ }^{\alpha}\left(\mathscr{A}_{H}\right)$ consists of an isolated collection of points. If all the critical points of $\mathscr{A}_{H}$ belonging to $P_{0}(X, L) \times \mathbb{R}$ are non-degenerate then $\mathscr{A}_{H}: P_{0}(X, L) \times \mathbb{R} \rightarrow \mathbb{R}$ is a Morse-Bott functional and $\operatorname{Crit}^{0}\left(\mathscr{A}_{H}\right)$ consists of an isolated collection of points and a copy of $\Sigma \cap L$.

Proof The fact that elements $(x, \tau) \in \mathrm{Crit}^{\alpha}\left(\mathscr{A}_{H}\right)$ with $\tau \neq 0$ are isolated follows easily from the bijectivity of $\nabla_{\mathbf{J}}^{2} \mathscr{A}_{H}(x, \tau)$. Set $\Sigma:=H^{-1}(0)$. Let us show that the set $\{(p, 0) \mid p \in \Sigma \cap L\}$ is a Morse-Bott component of $\mathrm{Crit}^{0}\left(\mathscr{A}_{H}\right)$. Since $\Sigma \pitchfork L$, it suffices to show that for all $p \in \Sigma \cap L$ we have

$$
\operatorname{ker} \nabla_{\mathbf{J}^{2}}^{2} \mathscr{A}_{H}(p, 0)=T_{p}(\Sigma \cap L) \times\{0\} \subset T_{(p, 0)}\left(P_{0}(X, L) \times \mathbb{R}\right) .
$$

If $p \in \Sigma \cap L$, an element $(\xi, h) \in T_{(p, 0)}\left(P_{0}(X, L) \times \mathbb{R}\right)$ is in the kernel of $\nabla_{\mathbf{J}}^{2} \mathscr{A}_{H}(p, 0)$ if and only if $(\xi, h)$ solves the equations:

$$
\begin{aligned}
& \dot{\xi}=h X_{H}(p), \\
& \int_{0}^{1} d H(x)(\xi) d t=0 .
\end{aligned}
$$

Integrating the first equation, we see that $\xi(t)=\xi(0)+h X_{H}(p)$. Since $\xi(0) \in T_{p} L$ and $X_{H}(p) \notin T_{p} L$ as $H^{-1}(0) \pitchfork L$, we must have $h=0$. Thus $\xi(t)=\xi(0)$ is constant. The second equation tells us that $d H(x)(\xi(0))=0$, and hence $\xi(0) \in T_{p} \Sigma$.

A standard Sard-Smale argument proves the following result (see for instance [43]):

Lemma 2.14 There is a generic subset of $C_{c t}^{\infty}(X, \mathbb{R})$ of Hamiltonians $H$ for which

1. 0 is a regular value of $H$,

2. $H^{-1}(0) \cap L \neq \varnothing$ and $H^{-1}(0) \pitchfork L$,

3. all the critical points of $\mathscr{A}_{H}$ belonging to $P_{\alpha}(X, L) \times \mathbb{R}$ are non-degenerate.

It is well known that if every critical point in $\operatorname{Crit}^{\alpha}\left(\mathscr{A}_{H}\right)_{a}^{b}$ is non-degenerate then for any choice of $\mathbf{J}=\left(J_{t}\right) \subset \mathscr{J}(X, \omega)$, every element $u \in \mathscr{M}^{\alpha}(H, \mathbf{J})_{a}^{b}$ is asymptotically convergent at each end to elements of $\mathrm{Crit}^{\alpha}\left(\mathscr{A}_{H}\right)_{a}^{b}$. That is, the limits

$$
\lim _{s \rightarrow \pm \infty} u(s, t)=\left(x_{ \pm}(t), \tau_{ \pm}\right), \quad \lim _{s \rightarrow \infty} \partial_{t} u(s, t)=0,
$$

exist, and the convergence is uniform in $t$, and the limits $\left(x_{ \pm}, \tau_{ \pm}\right)$belong to $\mathrm{Crit}^{\alpha}\left(\mathscr{A}_{H}\right)_{a}^{b}$ (see for instance [48]). If $E_{\mathbf{J}}(u)$ denotes the energy of a gradient flow line:

$$
E_{\mathbf{J}}(u):=\int_{-\infty}^{\infty}\left\|\partial_{s} u(s)\right\|_{\mathbf{J}}^{2} d s,
$$


then if $u \in \mathscr{M}^{\alpha}(H, \mathbf{J})_{a}^{b}$ is asymptotically convergent to $\left(x_{ \pm}, \tau_{ \pm}\right) \in \operatorname{Crit}\left(\mathscr{A}_{H}\right)_{a}^{b}$ one has

$$
E_{\mathbf{J}}(u)=\mathscr{A}_{H}\left(x_{-}, \tau_{-}\right)-\mathscr{A}_{H}\left(x_{+}, \tau_{+}\right),
$$

and hence $0 \leq E_{\mathbf{J}}(u) \leq b-a$.

Definition 2.15 We say that a Rabinowitz admissible triple $(\Sigma, L, \alpha)$ is a non-degenerate Rabinowitz admissible triple if for some (and hence any) $H \in \mathscr{D}_{\mathrm{ct}}(\Sigma)$, every critical point of $\mathscr{A}_{H}: P_{\alpha}(X, L) \times \mathbb{R} \rightarrow \mathbb{R}$ is non-degenerate.

Remark 2.16 In the simpler setting described in Remark 2.8 we equivalently say that $(\Sigma, L, \alpha)$ is non-degenerate if the Reeb chords of $\eta$ with endpoints in $K$ in the homotopy class $\alpha$ are isolated in $\Sigma$, and finally if $\zeta:[0, \tau] \rightarrow \Sigma$ is any such chord then

$$
D \eta^{\tau}(\zeta(0))\left(T_{\zeta(0)} L\right) \pitchfork T_{\zeta(\tau)} L .
$$

\subsection{Compactness}

Recall that an $\omega$-compatible almost complex structure $J$ is geometrically bounded if the corresponding Riemannian metric $g_{J}:=\omega(J \cdot, \cdot)$ is complete, has bounded sectional curvature and has injectivity radius bounded away from zero. By our initial assumption on $X$ such almost complex structures exist; let us fix once and for all such an almost complex structure $J_{\mathrm{gb}}$. We denote by $\mathscr{J}_{\mathrm{gb}}\left(X, \omega ; J_{\mathrm{gb}}\right) \subset \mathscr{J}(X, \omega)$ the set of almost complex structures $J \in \mathscr{J}(X, \omega)$ for which there exists a compact set $K \subset X$ (depending on $J$ ) such that $J=J_{\mathrm{gb}}$ on $X \backslash K$. Since in general it is unknown whether the set of all geometrically bounded almost complex structures on $(X, \omega)$ is connected, it is possible that everything we do will depend on our initial choice of geometrically bounded almost complex structure $J_{\mathrm{gb}}$. The general consensus however seems to be that this is unlikely. Regardless, we will ignore this subtlety throughout.

The following two compactness results are key to everything that follows. The first result is for gradient flow lines of a pair $(H, \mathbf{J})$; the second result is for $s$-dependent trajectories. These results were originally proved in the periodic case for hypersurfaces of restricted contact type in [20]. A full proof in this setting can be found in [38]. We remark that it is these results where the hypothesis that $\Sigma$ is of virtual restricted contact type is used, and where we use the fact that there exist good primitives of $\widetilde{\omega}$.

Theorem 2.17 Assume $(\Sigma, L, \alpha)$ is a non-degenerate Rabinowitz admissible triple. Let $H \in$ $\mathscr{D}_{\mathrm{ct}}(\Sigma)$ and $\mathbf{J}=\left(J_{t}\right) \subset \mathscr{J}_{\mathrm{gb}}\left(X, \omega ; J_{\mathrm{gb}}\right)$. Fix $-\infty<a<b<\infty$ and suppose $\left(u^{v}=\right.$ $\left.\left(x^{\nu}, \tau^{v}\right)\right)_{v \in \mathbb{N}} \subset \mathscr{M}^{\alpha}(H, \mathbf{J})_{a}^{b}$. Then for any sequence $\left(s^{\nu}\right) \subset \mathbb{R}$, the reparametrized sequence $u^{v}\left(\cdot+s^{v}\right)$ has a subsequence which converges in $C_{\mathrm{loc}}^{\infty}(\mathbb{R} \times[0,1], X) \times C_{\mathrm{loc}}^{\infty}(\mathbb{R}, \mathbb{R})$.

Recall the notion of a good homotopy from Definition 2.6.

Theorem 2.18 Assume $\left(\Sigma_{ \pm}, L, \alpha\right)$ are both non-degenerate Rabinowitz admissible triples. Fix Hamiltonians $H_{ \pm} \in \mathscr{D}_{\mathrm{ct}}\left(\Sigma_{ \pm}\right)$and suppose that there exists a good homotopy $\left(H_{s}, \lambda_{s}\right)_{s \in \mathbb{R}}$ such that $\left(H_{s}, \lambda_{s}\right)=\left(H_{-}, \lambda_{-}\right)$for $s \ll 0$ and $\left(H_{s}, \lambda_{s}\right)=\left(H_{+}, \lambda_{+}\right)$for $s \gg 0$, and such that $H_{s}$ has compact support uniformly in s. Fix $\mathbf{J}_{ \pm}=\left(J_{ \pm, t}\right) \subset \mathscr{J}_{\mathrm{gb}}\left(X, \omega ; J_{\mathrm{gb}}\right)$, and choose a smooth family $\left(\mathbf{J}_{s}=\left(J_{s, t}\right)\right)_{s \in \mathbb{R}} \subset \mathscr{J}_{\mathrm{gb}}\left(X, \omega ; J_{\mathrm{gb}}\right)$ such that there exists a compact set $K \subset X$ such that $J_{s, t}=J_{\mathrm{gb}}$ on $X \backslash K$ for all $(s, t) \in \mathbb{R} \times[0,1]$, and such that $\mathbf{J}_{s}=\mathbf{J}_{-}$for $s \ll 0$ and $\mathbf{J}_{s}=\mathbf{J}_{+}$for $s \gg 0$.

There exists a constant $\kappa>0$ such that if $\left\|\partial_{S} H_{S}\right\|_{L^{\infty}}<\kappa$ then the conclusion of the previous theorem holds. That is, if $\left\|\partial_{S} H_{s}\right\|_{L^{\infty}}<\kappa$ then for any sequence $\left(u^{v}=\left(x^{\nu}, \tau^{\nu}\right)\right)_{v \in \mathbb{N}} \subset$ 
$\mathscr{M}^{\alpha}\left(H_{s}, \mathbf{J}_{s}\right)_{a}^{b}$ and any sequence $\left(s^{\nu}\right) \subset \mathbb{R}$, the reparametrized sequence $u^{\nu}\left(\cdot+s^{\nu}\right)$ has a subsequence which converges in $C_{\mathrm{loc}}^{\infty}(\mathbb{R} \times[0,1], X) \times C_{\mathrm{loc}}^{\infty}(\mathbb{R}, \mathbb{R})$.

Remark 2.19 We remark that because we are assuming that all our Hamiltonians are constant outside of a compact set, the only thing one needs to prove in the above two theorems is that the Lagrange multiplier component $\tau$ of a flow line $u=(x, \tau)$ is uniformly bounded. The bound on the loop component $x$ comes essentially "for free" from our assumption that the almost complex structures we work with are all geometrically bounded outside of a compact set; see for instance [23]. Later on we will need to work with Hamiltonians that are not constant outside a compact set; hence more work will need to be done here (cf. the discussion in Sect. 3.2).

Remark 2.20 It follows from Theorem 2.17 that given $-\infty<a<b<\infty$, the subset $\mathrm{Crit}^{\alpha}\left(\mathscr{A}_{H}\right)_{a}^{b}$ is compact (by the Arzelà-Ascoli theorem). Thus as $\mathscr{A}_{H}$ is Morse [resp. Morse-Bott if $\alpha=0$ ], the set $\operatorname{Crit}^{\alpha}\left(\mathscr{A}_{H}\right)_{a}^{b}$ is at most finite [resp. has at most finitely many components].

\subsection{The definition of $\operatorname{RFH}_{*}^{\alpha}(H)$}

Assume $(\Sigma, L, \alpha)$ is a non-degenerate Rabinowitz admissible triple. In particular $\Sigma$ is transverse to $L$. The Rabinowitz action functional $\mathscr{A}_{H}$ is Morse-Bott by Lemma 2.13 (in fact, Morse if $\alpha \neq 0$ ). The aim of this subsection is introduce a Floer homology theory for the functional $\mathscr{A}_{H}$. There are various ways to deal with the problem that $\mathscr{A}_{H}: P_{0}(X, L) \times \mathbb{R} \rightarrow \mathbb{R}$ is not Morse. One possibility is to choose an additional small perturbation to make the Rabinowitz action functional a Morse functional. This was first done in a Floer homological setting by Pozniak in his thesis [45], and was carried out in the context of Rabinowitz Floer homology by Cieliebak et al. [22]. Another option is to introduce an auxiliary Morse function on $\mathrm{Crit}^{\alpha}\left(\mathscr{A}_{H}\right)$ and take as generators of the Rabinowitz Floer complex the critical points of the Morse function. This approach was studied originally by Frauenfelder [29] in the finite dimensional case, and also Bourgeois [18] and Bourgeois and Oancea [16] in the infinite dimensional case. Cieliebak and Frauenfelder used this construction in their original approach to Rabinowitz Floer homology (see [20, Appendix A]), and in this article we will do the same. We emphasize though that if $\alpha \neq 0$ then the Rabinowitz action functional is actually Morse, and in this case one can just work with normal gradient flow lines. Thus the reader should bear in mind that a lot of what follows can be considerably simplified when $\alpha \neq 0$.

Let $f: \operatorname{Crit}^{\alpha}\left(\mathscr{A}_{H}\right) \rightarrow \mathbb{R}$ denote a Morse function and a fix Riemannian metric $m$ on $\mathrm{Crit}^{\alpha}\left(\mathscr{A}_{H}\right)$ such that the flow $\varphi^{t}: \mathrm{Crit}^{\alpha}\left(\mathscr{A}_{H}\right) \rightarrow \mathrm{Crit}^{\alpha}\left(\mathscr{A}_{H}\right)$ of $-\nabla f:=-\nabla_{m} f$ is MorseSmale. We abbreviate

$$
\begin{aligned}
C^{\alpha}(f) & :=\operatorname{Crit}(f) \subset \operatorname{Crit}^{\alpha}\left(\mathscr{A}_{H}\right) \\
C^{\alpha}(f)_{a}^{b} & :=\operatorname{Crit}(f) \cap \operatorname{Crit}^{\alpha}\left(\mathscr{A}_{H}\right)_{a}^{b} .
\end{aligned}
$$

Note that $C^{\alpha}(f)_{a}^{b}$ is finite (cf. Remark 2.20). Fix $\mathbf{J}=\left(J_{t}\right) \subset \mathscr{J}_{\mathrm{gb}}\left(X, \omega ; J_{\mathrm{gb}}\right)$.

Definition 2.21 Fix $k \in \mathbb{N}$. Given $\left(x_{ \pm}, \tau_{ \pm}\right) \in C^{\alpha}(f)_{a}^{b}$, a flow line from $\left(x_{-}, \tau_{-}\right)$to $\left(x_{+}, \tau_{+}\right)$ with $k$ cascades is a $k$-tuple $(\mathbf{u}, \mathbf{T})=\left(\left(u_{j}\right)_{j=1, \ldots, k},\left(T_{j}\right)_{j=1, \ldots, k-1}\right)$ of gradient flow lines $u_{j}: \mathbb{R} \rightarrow P_{\alpha}(X, L) \times \mathbb{R}$ of $(H, \mathbf{J})$ and real numbers $T_{j} \geq 0$ such that

$$
\begin{aligned}
& \lim _{s \rightarrow-\infty} u_{1}(s) \in W^{u}\left(\left(x_{-}, \tau_{-}\right) ;-\nabla f\right), \quad \lim _{s \rightarrow+\infty} u_{k}(s) \in W^{s}\left(\left(x_{+}, \tau_{+}\right) ;-\nabla f\right), \\
& \lim _{s \rightarrow-\infty} u_{j+1}(s)=\varphi^{T_{j}}\left(\lim _{s \rightarrow \infty} u_{j}(s)\right) \text { for } j=1, \ldots, k-1 .
\end{aligned}
$$


We denote the space of gradient flow lines with $k$ cascades from the critical point $\left(x_{-}, \tau_{-}\right)$to the critical point $\left(x_{+}, \tau_{+}\right)$by $\widetilde{\mathscr{M}_{k}}\left(\left(x_{-}, \tau_{-}\right),\left(x_{+}, \tau_{+}\right)\right)$, and we denote by $\mathscr{M}_{k}\left(\left(x_{-}, \tau_{-}\right),\left(x_{+}, \tau_{+}\right)\right)$the quotient $\widetilde{\mathscr{M}_{k}}\left(\left(x_{-}, \tau_{-}\right),\left(x_{+}, \tau_{+}\right)\right) / \mathbb{R}^{k}$, where $\mathbb{R}^{k}$ acts by reparametrization on each of the $k$ cascades. We define a flow line with zero cascades to be a gradient flow line of $-\nabla f$, and denote by $\widetilde{\mathscr{M}_{0}}\left(\left(x_{-}, \tau_{-}\right),\left(x_{+}, \tau_{+}\right)\right)$the set of flow lines with zero cascades that are asymptotically equal to $\left(x_{ \pm}, \tau_{ \pm}\right)$. We put $\mathscr{M}_{0}\left(\left(x_{-}, \tau_{-}\right),\left(x_{+}, \tau_{+}\right)\right):=$ $\widetilde{\mathscr{M}_{0}}\left(\left(x_{-}, \tau_{-}\right),\left(x_{+}, \tau_{+}\right)\right) / \mathbb{R}$. Finally we define

$$
\mathscr{M}\left(\left(x_{-}, \tau_{-}\right),\left(x_{+}, \tau_{+}\right)\right):=\bigcup_{k \in \mathbb{N} \cup\{0\}} \mathscr{M}_{k}\left(\left(x_{-}, \tau_{-}\right),\left(x_{+}, \tau_{+}\right)\right) .
$$

Definition 2.22 Given a non-degenerate critical point $(x, \tau) \in \mathrm{Crit}^{\alpha}\left(\mathscr{A}_{H}\right)$ with $\tau \neq 0$, set

$$
\mu(x, \tau):=\mu_{\mathrm{Ma}}(x, \tau)-\frac{1}{2} \chi(x, \tau),
$$

where $\mu_{\mathrm{Ma}}(x, \tau)$ is the Maslov index of the path $\zeta(t):=x(t / \tau)$ (see [47] for the definition, and [38, Section 5.5] for the precise sign conventions we are using), and the correction term $\chi(x, \tau)$ was defined in (2.11). We set

$$
\mu(p, 0):=-\frac{n-1}{2} .
$$

If $(x, \tau) \in C^{\alpha}(f)$ define $\mu_{f}(x, \tau):=\mu(x, \tau)+i_{f}(x, \tau)$, where $i_{f}(x, \tau)$ is the Morse index of $(x, \tau)$ as a critical point of $f$ (thus $i_{f}(x, \tau)=0$ whenever $\tau \neq 0$ ). Our sign conventions imply that for all $(x, \tau) \in C^{\alpha}(f)$,

$$
\mu_{f}(x, \tau) \in \begin{cases}\mathbb{Z}, & \text { if } n \text { is odd, } \\ \frac{1}{2} \mathbb{Z} \backslash \mathbb{Z}, & \text { if } n \text { is even. }\end{cases}
$$

The following theorem is part of the standard Floer homology package, the key ingredient being Theorem 2.17. The index computation is probably the most non-routine element-full details of this aspect can be found in [38].

Theorem 2.23 For a generic choice of $\mathbf{J}$ and a generic Morse-Smale metric $m$ on Crit ${ }^{\alpha}\left(\mathscr{A}_{H}\right)$ the moduli spaces $\mathscr{M}\left(\left(x_{-}, \tau_{-}\right),\left(x_{+}, \tau_{+}\right)\right)$for $\left(x_{ \pm}, \tau_{ \pm}\right) \in C^{\alpha}(f)$ are smooth manifolds of finite dimension

$$
\operatorname{dim} \mathscr{M}\left(\left(x_{-}, \tau_{-}\right),\left(x_{+}, \tau_{+}\right)\right)=\mu_{f}\left(x_{-}, \tau_{-}\right)-\mu_{f}\left(x_{+}, \tau_{+}\right)-1 .
$$

Moreover if $\mu_{f}\left(x_{-}, \tau_{-}\right)=\mu_{f}\left(x_{+}, \tau_{+}\right)+1$ then $\mathscr{M}\left(\left(x_{-}, \tau_{-}\right),\left(x_{+}, \tau_{+}\right)\right)$is compact, and hence a finite set.

Denote by

$$
\operatorname{CRF}_{*}^{\alpha}(H, f)_{a}^{b}:=C_{*}^{\alpha}(f)_{a}^{b} \otimes \mathbb{Z}_{2},
$$

where the grading $*$ is given by the function $\mu_{f}$ from Definition 2.22. Given $\left(x_{ \pm}, \tau_{ \pm}\right) \in$ $C^{\alpha}(f)_{a}^{b}$ with $\mu_{f}\left(x_{-}, \tau_{-}\right)=\mu_{f}\left(x_{+}, \tau_{+}\right)+1$, we define the number $n\left(\left(x_{-}, \tau_{-}\right),\left(x_{+}, \tau_{+}\right)\right) \in$ $\mathbb{Z}_{2}$ to be the parity of the finite set $\mathscr{M}\left(\left(x_{-}, \tau_{-}\right),\left(x_{+}, \tau_{+}\right)\right)$. If $\left(x_{+}, \tau_{+}\right) \in C^{\alpha}(f)_{a}^{b}$ has $\mu_{f}\left(x_{-}, \tau_{-}\right) \neq \mu_{f}\left(x_{+}, \tau_{+}\right)+1$, set $n\left(\left(x_{-}, \tau_{-}\right),\left(x_{+}, \tau_{+}\right)\right)=0$. Now we define the boundary operator

$$
\partial_{a}^{b}=\partial_{a}^{b}(H, \mathbf{J}, f, m): \mathrm{CRF}_{*}^{\alpha}(H, f)_{a}^{b} \rightarrow \mathrm{CRF}_{*-1}^{\alpha}(H, f)_{a}^{b}
$$


as the linear extension of

$$
\left(x_{-}, \tau_{-}\right) \mapsto \sum_{\left(x_{+}, \tau_{+}\right) \in C(f)_{a}^{b}} n\left(\left(x_{-}, \tau_{-}\right),\left(x_{+}, \tau_{+}\right)\right)\left(x_{+}, \tau_{+}\right) .
$$

The usual argument shows that $\partial_{a}^{b} \circ \partial_{a}^{b}=0$, and thus $\left\{\mathrm{CRF}_{*}^{\alpha}(H, f)_{a}^{b}, \partial_{a}^{b}\right\}$ carries the structure of a differential $\mathbb{Z}_{2}$-vector space. We denote by $\mathrm{RFH}_{*}^{\alpha}(H, \mathbf{J}, f, m)_{a}^{b}$ its homology. The standard theory of continuation homomorphisms in Floer theory show that the homology $\mathrm{RFH}_{*}^{\alpha}(H, \mathbf{J}, f, m)_{a}^{b}$ is independent up to canonical isomorphism of the choices of $f, \mathbf{J}$, and $m$, and thus we omit them from the notation and write simply $\operatorname{RFH}_{*}^{\alpha}(H)_{a}^{b}$. Next, suppose $-\infty<a<a^{\prime}<b<\infty$. Let $\mathrm{p}_{a, a^{\prime}}^{b}: \mathrm{CRF}_{*}^{\alpha}(H, f)_{a}^{b} \rightarrow \mathrm{CRF}_{*}^{\alpha}(H, f)_{a^{\prime}}^{b}$ denote the projection along $\mathrm{CRF}_{*}^{\alpha}(H, f)_{a}^{a^{\prime}}$. Since the action decreases along gradient flow lines, $\mathrm{p}_{a, a^{\prime}}^{b}$ commutes with the boundary operators $\partial_{a}^{b}$ and $\partial_{a^{\prime}}^{b}$, and hence induces a map

$$
\mathrm{p}_{a, a^{\prime}}^{b}: \operatorname{RFH}_{*}^{\alpha}(H)_{a}^{b} \rightarrow \operatorname{RFH}_{*}^{\alpha}(H)_{a^{\prime}}^{b}
$$

Similarly given $-\infty<a<b<b^{\prime}<\infty$ the inclusion $C^{\alpha}(f)_{a}^{b} \hookrightarrow C^{\alpha}(f)_{a}^{b^{\prime}}$ induces maps

$$
i_{a}^{b, b^{\prime}}: \operatorname{RFH}_{*}^{\alpha}(H)_{a}^{b} \rightarrow \operatorname{RFH}_{*}^{\alpha}(H)_{a}^{b^{\prime}} .
$$

The complexes $\left\{\operatorname{RFH}_{*}^{\alpha}(H)_{a}^{b}, \mathrm{p}, i\right\}$ form a bidirect system of $\mathbb{Z}_{2}$-vector spaces, and hence we can define

$$
\operatorname{RFH}_{*}^{\alpha}(H):=\underset{a \downarrow-\infty b \uparrow \infty}{\lim } \underset{\leftarrow}{\lim } \operatorname{RFH}_{*}^{\alpha}(H)_{a}^{b}
$$

In fact, suppose that $\left(\Sigma_{ \pm}, L, \alpha\right)$ are both non-degenerate Rabinowitz admissible triples. Fix $H_{ \pm} \in \mathscr{D}_{\mathrm{ct}}\left(\Sigma_{ \pm}\right)$and suppose $\lambda_{ \pm}$are good primitives with respect to $\left(\Sigma_{ \pm}, L\right)$, and assume there exists a good homotopy $\left(H_{s}, \lambda_{s}\right)_{s \in \mathbb{R}}$ with $\left(H_{s}, \lambda_{s}\right)=\left(H_{-}, \lambda_{-}\right)$for $s \leq 0$ and $\left(H_{s}, \lambda_{s}\right)=$ $\left(H_{+}, \lambda_{+}\right)$for $s \geq 0$. Then one can prove

$$
\operatorname{RFH}_{*}^{\alpha}\left(H_{-}\right) \cong \operatorname{RFH}_{*}^{\alpha}\left(H_{+}\right) \text {. }
$$

This is a standard Floer theoretical argument, the key ingredient being Theorem 2.18. Details can be found in [38]. It follows in particular that if $H \in \mathscr{D}_{\mathrm{ct}}(\Sigma)$ then $\operatorname{RFH}_{*}^{\alpha}(H)$ depends only on $\Sigma, L, X$ and $\alpha$. Thus we can finally make the following definition:

Definition 2.24 If $(\Sigma, L, \alpha)$ is a non-degenerate Rabinowitz admissible triple, we define the Lagrangian Rabinowitz Floer homology of $(\Sigma, L, X, \alpha)$ by

$$
\mathrm{RFH}_{*}^{\alpha}(\Sigma, L, X):=\mathrm{RFH}_{*}^{\alpha}(H) \text { for any } H \in \mathscr{D}_{\mathrm{ct}}(\Sigma) .
$$

Moreover, since $\operatorname{RFH}_{*}^{\alpha}(H)$ is invariant under good homotopies we can define the Lagrangian Rabinowitz Floer homology $\operatorname{RFH}_{*}^{\alpha}(\Sigma, L, X)$ even when $(\Sigma, L, \alpha)$ is not nondegenerate, simply by first isotopying $\Sigma$ through good homotopies to a new hypersurface $\Sigma^{\prime}$ such that $\left(\Sigma^{\prime}, L, \alpha\right)$ is Rabinowitz admissible and non-degenerate (such a hypersurface $\Sigma^{\prime}$ exists by Lemma 2.14), and then defining

$$
\operatorname{RFH}_{*}^{\alpha}(\Sigma, L, X):=\operatorname{RFH}_{*}^{\alpha}\left(\Sigma^{\prime}, L, X\right) .
$$


2.4 Relative leaf-wise intersection points

Suppose $\psi: X \rightarrow X$ is a compactly supported Hamiltonian diffeomorphism, and let $L$ denote a Lagrangian submanifold of $X$, and $\Sigma$ a hypersurface whose intersection with $L$ is transverse and non-empty. Fix $H \in \mathscr{D}_{\mathrm{ct}}(\Sigma)$. Recall from the Introduction that a relative leaf-wise intersection point $p$ of $\psi$ is a point $p \in \Sigma \cap L$ such that the characteristic chord through $p$ intersects $\psi^{-1}(L)$. Equivalently, $p \in \Sigma \cap L$ is a point with the property that there exists $\tau \in \mathbb{R}$ such that

$$
\psi\left(\phi_{H}^{\tau}(p)\right) \in L
$$

Remark 2.25 It is of interest to know whether $\tau$ is uniquely determined by $p$. This could fail if \# $\left(\left\{\phi_{H}^{t}(p)\right\} \cap \psi^{-1}(L)\right)>1$ or if the orbit $\left\{\phi_{H}^{t}(p)\right\}_{t \in \mathbb{R}}$ is closed. However if $\operatorname{dim} X \geq 4$ and the hypersurface $\Sigma$ itself is non-degenerate (i.e. all periodic orbits of the flow $\phi_{H}^{t}$ are isolated on $\Sigma$ and after choosing a local transversal section to a periodic orbit the linearized flow along this periodic orbit has no eigenvalue equal to $1-$ see $[35$, Section 1$]$ for a precise definition) then for a generic choice of Hamiltonian diffeomorphism neither of these things happen. These statements are proved by arguing as in [4, Theorem 3.3] and [13, Lemma 8.2]. Since a generic hypersurface is non-degenerate (see e.g. [20, Appendix B]), it follows that if $\operatorname{dim} X \geq 4$ then 'generically' $\tau$ is uniquely determined by $p$.

Suppose $p$ is a relative leaf-wise intersection point for which the corresponding $\tau$ is uniquely determined. Write $\psi=\phi_{F}^{1}$ for $F_{t}: X \rightarrow \mathbb{R}$ a compactly supported Hamiltonian function. Consider the (not necessarily smooth) path $\zeta$ in $X$ which first travels from $x$ to $\phi_{H}^{\tau}(x)$ via $\phi_{H}^{t}$, and then travels from $\phi_{H}^{\tau}(x)$ to $\psi\left(\phi_{H}^{\tau}(x)\right)$ via $\phi_{F}^{t}$. Although $\zeta$ depends on the choice of Hamiltonians $H$ and $F$, the class $\alpha \in \Pi_{L}$ which $\zeta$ represents does not. This is a standard argument, which uses the fact any 1-periodic compactly supported Hamiltonian function on $X$ has at least one contractible periodic orbit in the interior of its support. Details can be found in several places; see for instance [49, Proposition 3.1] or [39, Lemma 3.7] (the latter reference deals specifically with leaf-wise intersection points). In either case we say that the relative leaf-wise intersection point belongs to the class $\alpha \in \Pi_{L}$.

We now recall from Sect. 1.2 how a suitable perturbation of the Rabinowitz action functional $\mathscr{A}_{H}$ gives rise to a new functional which detects the relative leaf-wise intersection points of $\psi$ which belong to a given $\alpha \in \Pi_{L}$. Let $\beta: S^{1} \rightarrow \mathbb{R}$ denote a smooth function with

$$
\beta(t)=0 \forall t \in\left[\frac{1}{2}, 1\right], \text { and } \int_{0}^{1} \beta(t) d t=1,
$$

and let $\chi:[0,1] \rightarrow[0,1]$ denote a smooth monotone map with $\chi\left(\frac{1}{2}\right)=0$ and $\chi(1)=1$. Fix $\alpha \in \Pi_{L}$ satisfying condition (A), as stated before Definition 2.7

$$
\mathscr{A}_{H}^{F}: P_{\alpha}(X, L) \times \mathbb{R} \rightarrow \mathbb{R}
$$

by

$$
\mathscr{A}_{H}^{F}(x, \tau):=\Omega(x)-\tau \int_{0}^{1} \beta(t) H(x(t)) d t-\int_{0}^{1} \dot{\chi}(t) F_{\chi(t)}(x(t)) d t .
$$

Denote by $\operatorname{Crit}^{\alpha}\left(\mathscr{A}_{H}^{F}\right)$ the set of critical points of $\mathscr{A}_{H}^{F}$. Note that a pair $(x, \tau)$ belongs to $\operatorname{Crit}\left(\mathscr{A}_{H}^{F}\right)$ if and only if: 


$$
\begin{aligned}
& \dot{x}=\tau \beta X_{H}(x)+\dot{\chi} X_{F_{\chi}}(x) ; \\
& \int_{0}^{1} \beta(t) H(x) d t=0 .
\end{aligned}
$$

Since $\beta(t) H(x)$ and $\dot{\chi}(t) F_{\chi(t)}(t, x)$ have disjoint time support, critical points of $\mathscr{A}_{H}^{F}$ first follow the flow of $\phi_{H}^{\int_{0}^{t} \beta(s) d s}$ in time [0, $\left.\frac{1}{2}\right]$ and then follow the flow of $\phi_{F}^{\chi(t)}$ in time $\left[\frac{1}{2}, 1\right]$. This leads to the following observation, which is proved in the same way as [3, Proposition 2.4], and explains why Lagrangian Rabinowitz Floer homology is useful in the study of relative leaf-wise intersection points.

Lemma 2.26 There is a surjective map

$e: \operatorname{Crit}^{\alpha}\left(\mathscr{A}_{H}^{F}\right) \rightarrow\{$ relative leaf-wise intersection points of $\psi$ belonging to $\alpha\}$

given by

$$
e(x, \tau):=x(0) .
$$

If $\operatorname{dim} X \geq 4$ then generically (in the sense of Remark 2.25), the map e is a bijection.

Definition 2.27 A critical point $(x, \tau) \in \mathrm{Crit}^{\alpha}\left(\mathscr{A}_{H}^{F}\right)$ is called non-degenerate if $\nabla_{\mathbf{J}}^{2} \mathscr{A}_{H}^{F}(x, \tau)$ is injective (cf. (2.8)).

The proof of the following result, which is the analogue of Lemma 2.14, is very similar to [4, Appendix A].

Theorem 2.28 There is a generic subset of $C_{0}^{\infty}\left(S^{1} \times X, \mathbb{R}\right)$ with the property that if $F_{t}$ belongs to this set then every critical point of the corresponding perturbed Rabinowitz action functional $\mathscr{A}_{H}^{F}$ is non-degenerate.

Suppose now that $(\Sigma, L, \alpha)$ is a Rabinowitz admissible triple, and $H \in \mathscr{D}_{\mathrm{ct}}(\Sigma)$. If every critical point of the perturbed Rabinowitz action functional $\mathscr{A}_{H}^{F}$ is non-degenerate, we can define the Lagrangian Rabinowitz Floer homology $\mathrm{RFH}_{*}^{\alpha}(H, F)$. This is defined in exactly the same way as before, only since we are now in a Morse situation, no additional Morse function $f$ is needed. Moreover, by choosing an $s$-dependent homotopy $F_{s}$ from $F$ to 0 , one sees that the usual continuation homomorphisms are well-defined and isomorphisms. Thus we conclude:

$$
\operatorname{RFH}_{*}^{\alpha}(H, F) \cong \mathrm{RFH}_{*}^{\alpha}(H)
$$

(see [4, Section 2.3] or [38, Section 6] for more information). In particular, if one can find a Hamiltonian diffeomorphism $\psi: X \rightarrow X$ such that there are no relative leaf-wise intersection points belonging to $\alpha$, then $\mathrm{Crit}^{\alpha}\left(\mathscr{A}_{H}^{F}\right)=\emptyset$ for any function $F_{t}$ such that $\psi=\phi_{F}^{1}$. In this case $\mathscr{A}_{H}^{F}$ is trivially Morse, and hence $\operatorname{RFH}_{*}^{\alpha}(H, F)$ is defined and equal to zero. We can now complete the proof of Theorem 1.11. Let us recall the statement (written more concisely).

Theorem 2.29 Assume that $(\Sigma, L, 0)$ is a Rabinowitz admissible triple. If there exists a compactly supported Hamiltonian diffeomorphism $\psi: X \rightarrow X$ with no relative leaf-wise intersection points (e.g. if one can displace $\Sigma$ from $L$ ) then there exists a characteristic chord in $\Sigma$ with endpoints in $\Sigma \cap L$. 
Proof Suppose there are no characteristic chords in $\Sigma$ with endpoints in $\Sigma \cap L$. Then $(\Sigma, L, 0)$ is trivially non-degenerate. Moreover for any $H \in \mathscr{D}_{\mathrm{ct}}(\Sigma)$ and any Morse function $f$ on $\operatorname{Crit}^{0}\left(\mathscr{A}_{H}\right) \cong \Sigma \cap L$ it is clear that the Rabinowitz Floer complex $\left\{\operatorname{CRF}_{*}^{0}(H, f), \partial\right\}$ reduces to the Morse complex $\left\{\mathrm{CM}_{*+(n-1) / 2}(f), \partial^{\text {Morse }}\right\}$, and thus the Rabinowitz Floer homology agrees with the Morse homology of $f$ (modulo a grading shift). In particular, it is non-zero. But now if there existed a compactly supported Hamiltonian diffeomorphism $\psi: X \rightarrow X$ with no relative leaf-wise intersection points belonging to 0 , then as we have just seen this would imply that $\mathrm{RFH}_{*}^{0}(\Sigma, L, X)=0$.

Remark 2.30 The proof of Theorem 1.3 goes along exactly the same lines, although note that in order to get started one first isotopes $L$ relative to $\partial X_{0}$ so that (2.6) is satisfied-this can be done without affecting whether $\Sigma$ is displaceable from $L$ or not.

\section{Computing the Lagrangian Rabinowitz Floer homology on twisted cotangent bundles}

We now move on to the setting of twisted cotangent bundles, with the aim of proving Theorem 1.13. As in the previous section, much of the following material can be simplified if the reader is only interested in Theorem 1.8, with as we will explain in Remark 3.7. We now recall the setup: let $M$ denote a closed connected orientable $n$-dimensional manifold, where $n \geq 2$. Let $\pi: T^{*} M \rightarrow M$ denote the footpoint map $\pi(q, p) \mapsto q$, and let $\rho: \widetilde{M} \rightarrow M$ denote the universal cover of $M$. We write $\rho_{\sharp}: T^{*} \widetilde{M} \rightarrow T^{*} M$ for the map defined by $\rho_{\sharp}(p):=\left(D \rho(q)^{-1}\right)^{*}(p)$ for $p \in T_{\rho(q)}^{*} \widetilde{M}$. Let $\lambda_{\text {can }} \in \Omega^{1}\left(T^{*} M\right)$ denote the Liouville 1form, defined by $\lambda_{\text {can }}=\sum_{j} p_{j} d q_{j}$ in local coordinates $(q, p)$. Suppose $\sigma \in \Omega^{2}(M)$ is a closed 2-form. We pull $\sigma$ back to $T^{*} M$ and add it to $d \lambda_{\text {can }}$ to obtain a new symplectic form

$$
\omega:=d \lambda_{\mathrm{can}}+\pi^{*} \sigma
$$

on $T^{*} M$. We will always insist that $\sigma$ is weakly exact, that is, the lift $\tilde{\sigma}:=\rho^{*} \sigma \in \Omega^{2}(\tilde{M})$ is exact (this is equivalent to requiring that $\left.\sigma\right|_{\pi_{2}(M)}=0$ ). In fact, we will always make the additional assumption that $\widetilde{\sigma}$ admits a bounded primitive: there exists $\varphi \in \Omega^{1}(\widetilde{M})$ such that $d \varphi=\widetilde{\sigma}$ and such that

$$
\sup _{q \in \widetilde{M}}\left|\varphi_{q}\right|<\infty,
$$

where the norm $|\cdot|$ is given by the lift of any Riemannian metric on $M$ to $\widetilde{M}$. When discussing cotangent bundles, it is more convenient to fix once and for all a point $\star \in M$ as a reference point, and then take $0_{\star} \in T_{\star}^{*} M$ to be our fixed reference point in $T^{*} M$. When discussing submanifolds $S$ of $M$, we always implicitly assume that $\star \in S$ (note this implies $0_{\star} \in N^{*} S$ ). We also fix a point $\widetilde{\star} \in \widetilde{M}$ that projects onto $\star$. We denote by $P(M, S)$ the space of smooth paths $q:[0,1] \rightarrow M$ with $q(0) \in S$ and $q(1) \in S$. We define $\Pi_{S}$ in exactly the same way as $\Pi_{L}$ was defined in (2.4), only with $M$ and $S$ replacing $X$ and $L$. Then $\Pi_{S}$ indexes the connected components of $P(M, S)$, and given $\alpha \in \Pi_{S}$ we let $P_{\alpha}(M, S)$ denote the corresponding connected component. If $x=(q, p) \in P\left(T^{*} M, N^{*} S\right)$ then $q \in P(M, S)$, and under the obvious identification $\Pi_{S} \cong \Pi_{N^{*} S}$, if $x \in P_{\alpha}\left(T^{*} M, N^{*} S\right)$ then $q \in P_{\alpha}(M, S)$. In particular, if we write our reference loops $x_{\alpha}$ as $\left(q_{\alpha}, p_{\alpha}\right)$, then $q_{\alpha}$ serves as a reference loop in $P_{\alpha}(M, S)$. One nice consequence of (3.1) is that every class $\alpha \in \Pi_{S}$ satisfies the condition (A), as stated before Definition 2.7 
Lemma 3.1 Suppose $S \subseteq M$ is a closed connected submanifold such that $\left.\sigma\right|_{S}=0$ and such that $N^{*} S$ is a virtually exact Lagrangian submanifold of $\left(T^{*} M, \omega_{\sigma}\right)$. Then for every path $f: S^{1} \times[0,1] \rightarrow T^{*} M$ with $f\left(S^{1} \times\{0,1\}\right) \subset N^{*} S$, one has $\int_{S^{1} \times[0,1]} f^{*} \omega=0$.

Proof The symplectic area functional $\Omega$ of $\left(X, \omega_{\sigma}\right)$ can be expressed as

$$
\Omega=\Omega_{\text {can }}+\pi^{*} \Omega_{\sigma},
$$

where

$$
\Omega_{\mathrm{can}}(x):=\int_{0}^{1} x^{*} \lambda_{\mathrm{can}},
$$

and $\Omega_{\sigma}$ is the $\sigma$-area defined by

$$
\Omega_{\sigma}(q):=\int_{[0,1] \times[0,1]} \bar{q}^{*} \sigma,
$$

where $\bar{q}$ is any filling of $q$ (i.e. any smooth map $\bar{q}:[0,1] \times[0,1] \rightarrow M$ with $\bar{q}(0, t)=q(t)$, $\bar{q}(1, t)=q_{\alpha}(t)$ and $\left.\bar{q}([0,1] \times\{0,1\}) \subset S\right)$. It thus suffices to show that if $f: S^{1} \times[0,1] \rightarrow$ $M$ satisfies $f\left(S^{1} \times\{0,1\}\right) \subset S$ then $\int_{S^{1} \times[0,1]} f^{*} \sigma=0$. Fix a bounded primitive $\varphi$ of $\widetilde{\sigma}$ with the property that $\left.\varphi\right|_{\widetilde{S}}=d s$ for some bounded function $s \in C^{\infty}(\widetilde{S}, \mathbb{R})$. Consider $G:=f_{*}\left(\pi_{1}\left(S^{1} \times[0,1]\right)\right) \leq \pi_{1}(M)$. Then $G$ is amenable, since $\pi_{1}\left(S^{1} \times[0,1]\right)=\mathbb{Z}$, which is amenable. Then [44, Lemma 5.3] tells us that since $\|\varphi\|_{L^{\infty}}<\infty$ and $\|s\|_{L^{\infty}}<\infty$, we can replace $\varphi$ by a $G$-invariant primitive $\varphi^{\prime}$ of $\widetilde{\sigma}$, and $s$ by a $G$-invariant function $s^{\prime}$ satisfying $\left.\varphi^{\prime}\right|_{\widetilde{S}}=d s^{\prime}$. Thus $\varphi^{\prime}$ and $s^{\prime}$ descend to define a primitive $\varphi^{\prime \prime} \in \Omega^{1}\left(S^{1} \times[0,1]\right)$ of $f^{*} \sigma$ and a function $s^{\prime \prime} \in C^{\infty}\left(S^{1} \times\{0,1\}, \mathbb{R}\right)$ with the property that $\left.\varphi^{\prime \prime}\right|_{S^{1} \times\{0,1\}}=d s^{\prime \prime}$. Hence by Stokes' Theorem, $\int_{S^{1} \times[0,1]} f^{*} \sigma=0$ as required.

\subsection{The Mañé critical value}

We now recall the definition of the critical value $c(H, \sigma)$, as introduced by Mañé in [36]. General references for the results stated below are [24, Proposition 2-1.1] or [19, Appendix A]. We then explain how to modify the definition of the critical value $c(H, \sigma)$ to take into account a given $\pi_{1}$-injective submanifold $S \subseteq M$ for which $\left.\sigma\right|_{S}=0$. This leads to a new critical value $c(H, \sigma, S)$. Fix an autonomous Tonelli Hamiltonian $H \in C^{\infty}\left(T^{*} M, \mathbb{R}\right)$, and denote by $\widetilde{H} \in C^{\infty}\left(T^{*} \widetilde{M}, \mathbb{R}\right)$ the lift of $H$ to the universal cover $T^{*} \widetilde{M}$. We define the Mañe critical value associated to $H$ and $\sigma$ by

$$
c(H, \sigma):=\inf _{\varphi} \sup _{q \in \widetilde{M}} \widetilde{H}\left(q,-\varphi_{q}\right)
$$

where the infimum is taken over all primitives $\varphi$ of $\widetilde{\sigma}$. Since $H$ is superlinear, $c(H, \sigma)<\infty$ if and only if $\widetilde{\sigma}$ admits a bounded primitive.

Remark 3.2 The strange looking sign convention in (3.2) is due to the fact that we are using the "unnatural" sign convention that the canonical symplectic form on $T^{*} M$ is given by $d \lambda_{\text {can }}$ (rather than $-d \lambda_{\text {can }}$ ).

Suppose now we bring into the picture a closed connected $\pi_{1}$-injective submanifold $S \subseteq M$ such that $\left.\sigma\right|_{S}=0$. Then we are only permitted to use primitives $\varphi$ of $\widetilde{\sigma}$ with the property that $\left.\varphi\right|_{\widetilde{S}}=d s$ for some bounded function $s: \widetilde{S} \rightarrow \mathbb{R}$. Taking the infimum over only 
these primitives we obtain a new critical value $c(H, \sigma, S)$. If no such primitives exist we set $c(H, \sigma, S)=\infty$. Clearly

$$
c(H, \sigma) \leq c(H, \sigma, S),
$$

and $c(H, \sigma, S)$ is finite if and only if $N^{*} S$ is virtually exact in the sense of Definition 2.4. Now recall from Sect. 1.1 the following definition:

Definition 3.3 Consider a closed connected hypersurface $\Sigma \subset T^{*} M$ and a closed connected submanifold $S \subseteq M$ such that $\left.\sigma\right|_{S}=0$, with $\Sigma \cap N^{*} S \neq \emptyset$ and $\Sigma \pitchfork N^{*} S$. The pair $(\Sigma, S)$ is called a Mañé supercritical pair if there exists a Tonelli Hamiltonian $H: T^{*} M \rightarrow \mathbb{R}$ with $c(H, \sigma, S)<0$, and such that $\Sigma$ is the regular level set $H^{-1}(0)$.

We now prove that Mañé supercritical pairs exactly fit into the framework of Theorem 1.11. Recall the notion of a Rabinowitz admissible triple from Definition 2.7.

Lemma 3.4 Suppose that $(\Sigma, S)$ is a Mañé supercritical pair. Then for any $\alpha \in \Pi_{S}$, the triple $\left(\Sigma, N^{*} S, \alpha\right)$ is a Rabinowitz admissible triple.

Proof First let us show that if $H$ is a Tonelli Hamiltonian such that $c(H, \sigma)<0$ then $\Sigma:=H^{-1}(0)$ is a hypersurface of virtual restricted contact type (cf. Definition 2.3). By the definition (3.2) of $c(H, \sigma)$ there exists $\varepsilon>0$ and a bounded primitive $\varphi$ of $\widetilde{\sigma}$ such that the lift $\widetilde{H}$ of $H$ satisfies $\widetilde{H}\left(q,-\varphi_{q}\right)<-\varepsilon$ for all $q \in \widetilde{M}$. Set $\lambda:=\widetilde{\lambda}_{\text {can }}+\widetilde{\pi}^{*} \varphi$, where $\widetilde{\lambda}_{\text {can }}$ is the Liouville 1 -form on $T^{*} \widetilde{M}$. Since $\varphi$ is bounded, we need only check that

$$
\inf _{(q, p) \in \widetilde{\Sigma}} \lambda\left(X_{\widetilde{H}}(q, p)\right)>0,
$$

where $X_{\widetilde{H}}$ is the symplectic gradient of $\widetilde{H}$ with respect to the lifted symplectic form $\widetilde{\omega}:=$ $d \widetilde{\lambda}_{\text {can }}+\widetilde{\pi}^{*} \widetilde{\sigma}$. Fix $(q, p) \in \widetilde{\Sigma}$, and let

$$
f(s):=\widetilde{H}\left(q,(1-s) \varphi_{q}+s p\right) .
$$

A simple computation yields

$$
\lambda\left(X_{\widetilde{H}}(q, p)\right)=f^{\prime}(1) .
$$

Now note that $f(0)<-\varepsilon$ and $f(1)=0$, and since $H$ is Tonelli, $f$ is convex and thus we must have $f^{\prime}(1)>\varepsilon$. This proves (3.3). Moreover if we assume the stronger assumption that $c(H, \sigma, S)<0$ then exactly the same argument shows that there exists good primitives (in the sense of Definition 2.5). Combined with Lemma 3.1 this completes the proof.

Example 3.5 Here is an example (due to Alberto Abbondandolo) that illustrates the difference between simply asking that $c(H, \sigma)<0$ and asking that $c(H, \sigma, S)<0$. Take $M=\mathbb{T}^{n}$ and $\sigma=0$, and take $S=S^{1} \times\{\mathrm{pt}\}$. Define $H: T^{*} \mathbb{T}^{n} \rightarrow \mathbb{R}$ by

$$
H(q, p):=\frac{1}{2}\left|p-d q_{1}\right|^{2} .
$$

One easily sees that

$$
c(H, \sigma)=0
$$

but that

$$
c(H, \sigma, S)=1 / 2 .
$$

In fact, $H^{-1}(k) \cap N^{*} S=\emptyset$ if $k<1 / 2$. For $k>1 / 2$, not only is $H^{-1}(k) \cap N^{*} S$ non-empty, but it follows from Theorem 1.13 that the hypersurface $H^{-1}(k)$ can never be displaced from $N^{*} S$ by an element of $\operatorname{Ham}_{c}\left(T^{*} M, d \lambda_{\text {can }}\right)$. 


\subsection{Lagrangian Rabinowitz Floer homology with Tonelli Hamiltonians}

Since from now on we will be working exclusively with Mañé supercritical pairs, it would be nice to work directly with the Tonelli Hamiltonian $H$ which cuts $\Sigma$ out as its regular level set $H^{-1}(0)$. Such a Tonelli Hamiltonian $H$ belongs to $\mathscr{D}(\Sigma)$, but since Tonelli Hamiltonians are not constant outside a compact set, it does not belong to $\mathscr{D}_{\mathrm{ct}}(\Sigma)$. Thus it is not a priori clear that one can use $H$ to define the Lagrangian Rabinowitz Floer homology of $\left(\Sigma, N^{*} S, T^{*} M\right)$, and even if we could, whether it would yield the same Lagrangian Rabinowitz Floer homology as the one developed in Sect. 2.3. The key difficulty here is that as $H$ is no longer constant outside a compact set, a lot more work is required to prove the the compactness results in Theorem 2.17 and Theorem 2.18 (see Remark 2.19). In [9], Abbondandolo and Schwarz showed how such compactness could still be obtained (in the setting of "standard" Floer homology on cotangent bundles equipped with standard symplectic form $d \lambda_{\text {can }}$ ) for a wide class of Hamiltonians. Roughly speaking, they proved $L^{\infty}$ estimates for Hamiltonians that, outside of a compact set, are quadratic in the fibres (see [9, Section 1.5] for the precise definition). Their idea is based upon isometrically embedding $T^{*} M$ into $\mathbb{R}^{2 N}$ (via Nash's theorem), and combining Calderon-Zygmund estimates for the Cauchy-Riemann operator with certain interpolation inequalities. We remark that in order for these $L^{\infty}$ estimates to hold it is important that the almost complex structure we choose lies sufficiently close (in the $L^{\infty}$ norm) to the metric almost complex structure $J_{g}$ associated to some fixed Riemannian metric $g=\langle\cdot, \cdot\rangle$ on $M$. This is the unique almost complex structure on $T^{*} M$ with the property that under the splitting $T T^{*} M \cong T M \oplus T^{*} M$ determined by the metric (see Sect. 3.4 below), $J_{g}$ acts as

$$
J_{g}=\left(\begin{array}{cc}
0 & -\mathbb{I} \\
\mathbb{I} & 0
\end{array}\right)
$$

A Tonelli Hamiltonian $H \in C^{\infty}\left(T^{*} M, \mathbb{R}\right)$ is electromagnetic at infinity (with respect to $g$ ) if there exists a positive function $a \in C^{\infty}\left(M, \mathbb{R}^{+}\right)$, a 1 -form $\beta \in \Omega^{1}(M)$, a function $V \in C^{\infty}(M, \mathbb{R})$, and a real number $R>0$ such that

$$
H(q, p)=\frac{1}{2} a(q)\left|p-\beta_{q}\right|^{2}+V(q) \text { for all }(q, p) \in T^{*} M \text { with }|p| \geq R .
$$

The following result is a minor variant of [26, Corollary 20].

Proposition 3.6 Suppose $\Sigma=H^{-1}(0)$ is a regular energy value of a Tonelli Hamiltonian $H \in C^{\infty}\left(T^{*} M, \mathbb{R}\right)$ with $c(H, \sigma, S)<0$. Then there exists another Tonelli Hamiltonian $\bar{H}$ that is electromagnetic at infinity and satisfies:

$$
\begin{aligned}
& H \equiv \bar{H} \text { on }\{H \leq 1\} ; \\
& c(H, \sigma, S)=c(\bar{H}, \sigma, S) .
\end{aligned}
$$

In [38] we use a version of the argument of Abbondandolo and Schwarz mentioned above to show that the Lagrangian Rabinowitz Floer homology $\operatorname{RFH}_{*}^{\alpha}(H)$ is well defined when $H$ is a Tonelli Hamiltonian which is electromagnetic at infinity and satisfies $c(H, \sigma, S)<0$, and moreover that this Lagrangian Rabinowitz Floer homology is the same as the one defined using Hamiltonians which are constant outside a compact set. Actually strictly speaking in order for this result to hold, one may need to rescale $\sigma$ (this is so $\omega$-compatible almost complex structures that are sufficiently close in the $L^{\infty}$-norm to the metric almost complex structure $J_{g}$ exist); this does not actually entail any loss of generality, as the Lagrangian 
Rabinowitz Floer homology of Sect. 2.3 is invariant under such rescaling. As such we will ignore this subtlety throughout. See [38, Lemma 8.12].

From now on we fix a Mañé supercritical pair $(\Sigma, S)$. Without loss of generality (as far as the Lagrangian Rabinowitz Floer homology is concerned), we can and will assume that $\left(\Sigma, N^{*} S, \alpha\right)$ is non-degenerate for every $\alpha \in \Pi_{S}$. Proposition 3.6 implies that we may choose a Tonelli Hamiltonian $H \in C^{\infty}\left(T^{*} M, \mathbb{R}\right)$ that is electromagnetic at infinity and satisfies $\Sigma=H^{-1}(0)$ with $c(H, \sigma, S)<0$, and thus we may compute the Lagrangian Rabinowitz Floer homology $\mathrm{RFH}_{*}^{\alpha}\left(\Sigma, N^{*} S, T^{*} M\right)$ using $H$ :

$$
\operatorname{RFH}_{*}^{\alpha}(H) \cong \operatorname{RFH}_{*}^{\alpha}\left(\Sigma, N^{*} S, T^{*} M\right) .
$$

The aim of the rest of this paper is to compute $\operatorname{RFH}_{*}^{\alpha}(H)$.

Remark 3.7 For the reader only interested in Theorem 1.8, where $\sigma=0$ and $\Sigma=U^{*} M$, we can drop the assumption that $S$ is $\pi_{1}$-injective, since we no longer need to lift anything to the universal cover. For our Hamiltonian $H$ we take $H(q, p)=\frac{1}{2}|p|^{2}-\frac{1}{2}$. In this case for any submanifold $S \subseteq M$ one has $c(H, 0, S)=-\frac{1}{2}$. Thus (so far) there are no restrictions on $S$ apart from our standing assumption that $U^{*} M \cap N^{*} S \neq \emptyset$ and $U^{*} M \pitchfork N^{*} S$, although as stated in Theorem 1.8 we will eventually need to make an additional assumption on $S$ in order to compute the Lagrangian Rabinowitz Floer homology—see Theorem 3.16.

\subsection{Grading}

Before getting started on computing $\mathrm{RFH}_{*}^{\alpha}(H)$, we will spend a little time discussing the grading on Lagrangian Rabinowitz Floer homology in the specialized situation we are now working in. In fact, there is a particularly satisfying solution to the grading issue on twisted cotangent bundles. This is because every twisted cotangent bundle possesses a Lagrangian distribution, namely the vertical distribution $T^{\vee} T^{*} M$ (i.e. the tangent spaces to the fibres: $\left.T_{(q, p)}^{\mathrm{v}} T^{*} M:=T_{(q, p)} T_{q}^{*} M\right)$. The vertical distribution singles out a distinguished class of symplectic trivializations - those that are vertical preserving. Namely, if $x \in P\left(T^{*} M, N^{*} S\right)$, a trivialization $\Phi:[0,1] \times \mathbb{R}^{2 n} \rightarrow x^{*} T T^{*} M$ is called vertical preserving if

$$
\Phi\left(t, V_{0}\right)=T_{x(t)}^{\mathrm{v}} T^{*} M \text { for all } t \in[0,1],
$$

where $V_{0}:=\{0\} \times \mathbb{R}^{n}$ is the vertical subspace. Such trivializations always exist (cf. [9, Lemma 1.2]). Suppose we are given a critical point $(x, \tau)$ of the Rabinowitz action functional $\mathscr{A}_{H}$. Let $\Phi:[0,1] \times \mathbb{R}^{2 n} \rightarrow x^{*} T T^{*} M$ denote a vertical preserving trivialization, and define a path $\vartheta:[0,1] \rightarrow \operatorname{Lag}\left(\mathbb{R}^{2 n}, \omega_{\text {std }}\right)$ by

$$
\Phi(t, \vartheta(t))=D \phi_{H}^{\tau t}(x(0))\left(T_{x(0)}^{\vee} T^{*} M\right) .
$$

Now define

$$
\mu_{\mathrm{Ma}}(x, \tau):=\mu_{\mathrm{RS}}\left(\vartheta, V_{0}\right),
$$

where $\mu_{\mathrm{RS}}$ is the Robbin-Salamon index [47] (although be warned-our sign convention for $\mu_{\mathrm{RS}}$ matches [7] rather than [47]). This index $\mu_{\mathrm{Ma}}(x, \tau)$ is independent of the vertical preserving trivialization $\Phi$ (cf. [9, Lemma 1.3.(ii)]). In fact it will also be convenient to introduce a grading shift of $d-\frac{n-1}{2}($ recall $d=\operatorname{dim} S)$. This choice is motivated by Theorem 3.14 below, and it also ensures our grading is always $\mathbb{Z}$-valued. Thus for the remainder of the paper, instead of using the convention from Definition 2.22 we define

$$
\mu(x, \tau):= \begin{cases}\mu_{\mathrm{Ma}}(x, \tau)-\frac{1}{2} \chi(x, \tau)+d-\frac{n-1}{2}, & \tau \neq 0 \\ d-n+1, & \tau=0 .\end{cases}
$$


3.4 The free time action functional

In this section we work on the tangent bundle $T M$ instead of the cotangent bundle $T^{*} M$. Denote again by $\pi$ the footpoint map $T M \rightarrow M$. Let us fix once and for all an auxiliary Riemannian metric $g$ on $M$. The Riemannian metric $g$ defines a horizontal-vertical splitting of $T T M$ : given $(q, v) \in T M$ we write

$$
T_{(q, v)} T M=T_{(q, v)}^{\mathrm{h}} T M \oplus T_{(q, v)}^{\mathrm{v}} T M \cong T_{q} M \oplus T_{q} M
$$

here $T_{(q, v)}^{\mathrm{h}} T M=\operatorname{ker}\left(\kappa_{g}: T_{(q, v)} T M \rightarrow T_{q} M\right)$, where $\kappa_{g}$ is the connection map of the Levi-Civita connection $\nabla$ of $g$, and $T_{(q, v)}^{\mathrm{v}} T M=\operatorname{ker}\left(D \pi(q, v): T_{(q, v)} T M \rightarrow T_{q} M\right)$. Given $\xi \in T T M$ we denote by $\xi^{\mathrm{h}}$ and $\xi^{\vee}$ the horizontal and vertical components. The Sasaki metric $g_{T M}$ on $T M$ is defined by

$$
g_{T M}(\xi, \vartheta):=\left\langle\xi^{\mathrm{h}}, \vartheta^{\mathrm{h}}\right\rangle+\left\langle\xi^{\mathrm{v}}, \vartheta^{\mathrm{v}}\right\rangle .
$$

Suppose $f \in C^{\infty}(T M, \mathbb{R})$ is an arbitrary smooth function. Then $d f(q, v) \in T_{(q, v)}^{*} T M$, and thus its gradient $\nabla f(q, v)=\nabla_{g_{T M}} f(q, v)$ lies in $T_{(q, v)} T M$. Thus we can speak of the horizontal and vertical components

$$
\begin{aligned}
& \nabla f^{\mathrm{h}}(q, v):=[\nabla f(q, v)]^{\mathrm{h}} \in T_{q} M ; \\
& \nabla f^{\mathrm{v}}(q, v):=[\nabla f(q, v)]^{\mathrm{v}} \in T_{q} M .
\end{aligned}
$$

Let us go back to our fixed Hamiltonian $H$. The fact that $H$ is Tonelli implies there exists a unique Tonelli Lagrangian (that is, fibrewise strictly convex and superlinear) $L \in C^{\infty}(T M, \mathbb{R})$ called the Fenchel dual Lagrangian to $H$, which is related to $H$ by

$$
H(q, p):=p(v)-L(q, v), \text { where } \nabla L^{\mathrm{v}}(q, v)=p .
$$

Since $H$ is electromagnetic at infinity, so is $L$. That is, there exists a positive function $a \in C^{\infty}\left(M, \mathbb{R}^{+}\right)$, a 1 -form $\beta \in \Omega^{1}(M)$, a function $V \in C^{\infty}(M, \mathbb{R})$, and a real number $R>0$

$$
L(q, v)=\frac{1}{2} a(q)|v|^{2}+\beta_{q}(v)-V(q) \text { for all }(q, v) \in T M \text { with }|p| \geq R .
$$

Definition 3.8 We denote by $\mathscr{P}(M, S)$ the the $W^{1,2}$ Sobolev completion of $P(M, S)$. Abbreviating $\mathbb{R}^{+}:=(0, \infty)$, we equip $\mathscr{P}(M, S) \times \mathbb{R}^{+}$with the natural product Riemannian structure

$$
\left\langle\left\langle(\eta, h),\left(\eta^{\prime}, h^{\prime}\right)\right\rangle\right\rangle_{W^{1,2}}:=\int_{0}^{1}\left\langle\eta, \eta^{\prime}\right\rangle d t+\int_{0}^{1}\left\langle\nabla_{t} \eta, \nabla_{t} \eta^{\prime}\right\rangle d t+h h^{\prime},
$$

where $\nabla$ denotes the Levi-Civita connection of $(M, g)$.

Recall from the proof of Lemma 3.1 that $\sigma$-area $\Omega_{\sigma}: P(M, S) \rightarrow \mathbb{R}$ is defined by

$$
\Omega_{\sigma}(q):=\int_{[0,1] \times[0,1]} \bar{q}^{*} \sigma,
$$

where $q \in P_{\alpha}(M, S)$ and $\bar{q}$ is any filling of $q$ (i.e. any smooth map such that $\bar{q}(0, t)=q(t)$, $\bar{q}(1, t)=q_{\alpha}(t)$ and $\left.\bar{q}([0,1] \times\{0,1\}) \subset S\right)$. Let us note that $\Omega_{\sigma}$ extends to a functional on 
$\mathscr{P}(M, S)$ : if $q$ is of class $W^{1,2}$ then we choose the filling $\bar{q} \in W^{1,2}([0,1] \times[0,1], M) \cap$ $C^{0}([0,1] \times[0,1], M)$. We will study the free time action functional

$$
\mathscr{S}_{L}: \mathscr{P}_{\alpha}(M, S) \times \mathbb{R}^{+} \rightarrow \mathbb{R}
$$

which is defined by

$$
\mathscr{S}_{L}(q, \tau):=\Omega_{\sigma}(q)+\tau \int_{0}^{1} L\left(q, \frac{\dot{q}}{\tau}\right) d t .
$$

In the case $\sigma=0$, the functional $\mathscr{S}_{L}$ has been extensively studied in [26,27]. The pair $(\sigma, g)$ defines a bundle endomorphism $Y=Y_{\sigma, g} \in \Gamma(\operatorname{End}(T M))$ called the Lorentz force of $\sigma$ via:

$$
\sigma_{q}(u, v)=\langle Y(q) u, v\rangle .
$$

A standard computation tells us that if $(q, \tau) \in \mathscr{P}_{\alpha}(M, S) \times \mathbb{R}^{+}$and $\left(q_{s}, \tau_{s}\right)_{s \in(-\varepsilon, \varepsilon)} \subset$ $\mathscr{P}_{\alpha}(M, S) \times \mathbb{R}^{+}$is a variation of $(q, \tau)$ of class $C^{2}$ with $\left.\frac{\partial}{\partial s}\right|_{s=0} q_{s}(t)=: \eta(t)$ and $\left.\frac{\partial}{\partial s}\right|_{s=0} \tau_{s}=$ : $h$ then setting $\gamma(t):=q(t / \tau)$ and $v(t):=\eta(t / \tau)$ one has

$$
\begin{aligned}
\left.\frac{\partial}{\partial s}\right|_{s=0} \mathscr{S}_{L}\left(q_{s}, \tau_{s}\right)= & \int_{0}^{\tau}\left\langle\nabla L^{\mathrm{h}}(\gamma, \dot{\gamma})-\nabla_{t}\left(\nabla L^{\mathrm{v}}(\gamma, \dot{\gamma})\right)-Y(\gamma) \dot{\gamma}, v\right\rangle d t, \\
& -\frac{h}{\tau} \int_{0}^{\tau} E(\gamma, \dot{\gamma}) d t+\left[\left\langle\nabla L^{\mathrm{v}}(\gamma(1), \dot{\gamma}(1), v(1)\rangle-\left\langle\nabla L^{\mathrm{v}}(\gamma(0), \dot{\gamma}(0), v(0)\rangle\right],\right.\right.
\end{aligned}
$$

where $E: T M \rightarrow \mathbb{R}$ is defined by

$$
E(q, v):=H\left(\nabla L^{\mathrm{v}}(q, v)\right) .
$$

Thus $\left.\frac{\partial}{\partial s}\right|_{s=0} \mathscr{S}_{L}\left(q_{s}, \tau_{s}\right)=0$ for all such variations $\left(q_{s}, \tau_{s}\right)$ if and only if $\gamma(t):=q(t / \tau)$ satisfies the Euler-Lagrange equations

$$
\nabla L^{\mathrm{h}}(\gamma, \dot{\gamma})-\nabla_{t}\left(\nabla L^{\mathrm{v}}(\gamma, \dot{\gamma})\right)-Y(\gamma) \dot{\gamma}=0
$$

together with the energy constraint

$$
\int_{0}^{1} E(\gamma, \dot{\gamma}) d t=0
$$

and the boundary conditions

$$
\left\langle\nabla L^{\mathrm{v}}(\gamma(1), \dot{\gamma}(1), u\rangle=\left\langle\nabla L^{\mathrm{v}}(\gamma(0), \dot{\gamma}(0), v\rangle \quad \text { for all } u \in T_{\gamma(0)} S \text { and } v \in T_{\gamma(1)} S .\right.\right.
$$

In particular, note that any critical point $(q, \tau)$ of $\mathscr{S}_{L}$ actually belongs to $P_{\alpha}(M, S) \times \mathbb{R}^{+}$(i.e. $q$ is smooth). Since $L$ is electromagnetic at infinity, $\mathscr{S}_{L}$ is of class $C^{1,1}$ on $\mathscr{P}_{\alpha}(M, S) \times \mathbb{R}^{+}$ (see $[10,11]$ ). It will also be useful to consider the fixed period action functional. Given $\tau \in \mathbb{R}^{+}$let us denote by $\mathscr{S}_{L}^{\tau}$ the functional defined by

$$
\mathscr{S}_{L}^{\tau}(q):=\mathscr{S}_{L}(q, \tau) .
$$

Note that

$$
d \mathscr{S}_{L}^{\tau}(q)(\eta)=d \mathscr{S}_{L}(q, \tau)(\eta, 0)
$$


Thus if $(q, \tau) \in \mathrm{Crit}^{\alpha}\left(\mathscr{S}_{L}\right)$ then $q \in \mathrm{Crit}^{\alpha}\left(\mathscr{S}_{L}^{\tau}\right)$. By definition, the Morse index $m(q, \tau)$ of a critical point $(q, \tau) \in \mathrm{Crit}^{\alpha}\left(\mathscr{S}_{L}\right)$ is the maximal dimension of a subspace $W^{1,2}\left(q^{*} T M\right) \times \mathbb{R}$ on which the Hessian $\nabla^{2} \mathscr{S}_{L}(q, \tau)$ of $\mathscr{S}_{L}$ at $(q, \tau)$ is negative definite. Similarly we denote by $m_{\tau}(q)$ the Morse index of a critical point $q \in \mathrm{Crit}^{\alpha}\left(\mathscr{S}_{L}^{\tau}\right)$, that is, the maximal dimension of a subspace of $W^{1,2}\left(q^{*} T M\right)$ on which the Hessian $\nabla^{2} \mathscr{S}_{L}^{\tau}$ of $\mathscr{S}_{L}^{\tau}$ is negative definite. It is well known that for Tonelli Lagrangians the Morse index $m_{\tau}(q)$ is always finite [28, Section 1]. We define the nullity $n(q, \tau)$ of a critical point of $\mathscr{S}_{L}$ to be

$$
n(q, \tau):=\operatorname{dim} \operatorname{ker}\left(\nabla_{q}^{2} \mathscr{S}_{L}^{\tau}\right),
$$

and we say that a critical point $(q, \tau) \in \mathscr{P}_{\alpha}(M, S) \times \mathbb{R}^{+}$is non-degenerate if $n(q, \tau)=0$. Since we have assumed that our fixed Hamiltonian $H$ is non-degenerate, it actually follow that every critical point of $\mathscr{S}_{L}$ is non-degenerate. This is because there is a simple relationship between the critical points of $\mathscr{S}_{L}$ and those of $\mathscr{A}_{H}$, which we will discuss further in Lemma 3.13 below. Moreover Lemma 3.13, together with the discussion on page 13, implies that for each critical point $(q, \tau)$ of $\mathscr{S}_{L}$, there exists a unique family $\left(q_{s}, \tau(s)\right) \in \operatorname{Crit}\left(\mathscr{S}_{L+e(s)}\right)$ for $s \in(-\varepsilon, \varepsilon)$, where $\left(q_{0}, \tau(0)\right)=(q, \tau)$ and $e(0)=0$. Moreover we have $\tau^{\prime}(0) \neq 0$ and $e^{\prime}(0) \neq 0$. We can therefore define the correction term:

$$
\chi(q, \tau):=\operatorname{sign}\left(-\frac{e^{\prime}(0)}{\tau^{\prime}(0)}\right) \in\{-1,1\} .
$$

A proof of the following result can be found in [38, Section 10.2] (see also [42, Theorem 1.2]).

Theorem 3.9 Let $(q, \tau) \in \operatorname{Crit}^{\alpha}\left(\mathscr{S}_{L}\right)$. Then

$$
m(q, \tau)=m_{\tau}(q)+\frac{1}{2}-\frac{1}{2} \chi(q, \tau) .
$$

3.5 The Palais-Smale condition

Work of Abbondandolo and Schwarz [10,11] implies that we can find a smooth bounded vector field $\mathbf{G}$ on $\mathscr{P}_{\alpha}(M, S) \times \mathbb{R}^{+}$with the following two properties:

- There exists a continuous function $\delta \in C^{\infty}\left(\mathscr{P}_{\alpha}(M, S) \times \mathbb{R}^{+}, \mathbb{R}\right)$ such that for all $(q, \tau) \in$ $\mathscr{P}_{\alpha}(M, S) \times \mathbb{R}^{+}$one has

$$
d \mathscr{S}_{L}(q, \tau)(\mathbf{G}(q, \tau)) \geq \delta\left(\mathscr{S}_{L}(q, \tau)\right)\left\|d \mathscr{S}_{L}(q, \tau)\right\|_{W^{1,2}} .
$$

- For each $(q, \tau) \in \mathscr{P}_{\alpha}(M, S) \times \mathbb{R}^{+}$one has

$$
\mathscr{S}_{L}(q, \tau) \in \operatorname{Crit}^{\alpha}\left(\mathscr{S}_{L}\right) \Leftrightarrow \mathbf{G}(q, \tau)=0,
$$

and moreover if $(q, \tau) \in \operatorname{Crit}\left(\mathscr{S}_{L}\right)$ then

$$
\nabla^{2} \mathscr{S}_{L}(q, \tau)=\nabla \mathbf{G}(q, \tau)
$$

where $\nabla \mathbf{G}(q, \tau)$ denotes the Jacobian of $\mathbf{G}$, defined by $\nabla \mathbf{G}(q, \tau)(\xi, h):=[\mathbf{G}, V](q, \tau)$, with $V$ any vector field on $\mathscr{P}_{\alpha}(M, S) \times \mathbb{R}^{+}$such that $V(q, \tau)=(\xi, h)$.

Moreover in the case $\alpha=0$, we may additionally insist that the following two properties hold:

- There exists $k_{1}>0$ such that

$$
\left\langle\left.\left\langle\mathbf{G}(q, \tau), \frac{\partial}{\partial \tau}\right\rangle\right|_{W^{1,2}}<0 \text { if } \mathscr{S}_{L}(q, \tau) \geq k_{1} \tau\right.
$$

(see [10, Section 11] or [38, Lemma 10.3]). 
- If $\Upsilon^{s}$ denotes the local flow of - G then the submanifold $S \times \mathbb{R}^{+} \subset \mathscr{P}_{0}(M, S) \times \mathbb{R}^{+}$of constant curves is invariant under $\Upsilon^{s}$, that is, whenever defined one has

$$
\Upsilon^{s}\left(S \times \mathbb{R}^{+}\right) \subset S \times \mathbb{R}^{+}
$$

We shall refer to a smooth bounded vector field $\mathbf{G}$ that satisfies these four properties as a refined pseudo-gradient for $\mathscr{S}_{L}$. For a given point $(q, \tau) \in \mathscr{P}_{\alpha}(M, S) \times \mathbb{R}^{+}$, we denote by $\left(\omega_{-}(q, \tau), \omega_{+}(q, \tau)\right) \subset \mathbb{R}$ the maximal interval of existence of the flow line $s \mapsto \Upsilon^{s}(q, \tau)$. The next result is the key to defining the Morse (co)complex of $\mathscr{S}_{L}$ (compare [10, Proposition 11.1, Proposition 11.2]). A full proof in our setting is given in [38].

Theorem 3.10 Let $\mathbf{G}$ denote a refined pseudo-gradient for $\mathscr{S}_{L}$, and let $\Upsilon^{s}$ denote the local flow of -G. Then:

1. If $\alpha \neq 0$ then the pair $\left(\mathscr{S}_{L}, \mathbf{G}\right)$ satisfies the Palais-Smale condition at the level a for all $a \in \mathbb{R}$. If $\alpha=0$ then the pair $\left(\mathscr{S}_{L}, \mathbf{G}\right)$ satisfies the Palais-Smale condition at the level a for all $a \in \mathbb{R} \backslash\{0\}$.

2. $\mathscr{S}_{L}$ is bounded below on $\mathscr{P}_{\alpha}(M, S) \times \mathbb{R}^{+}$.

3. If $\alpha \neq 0$ then $\omega_{+}(q, \tau)=\infty$ for all $(q, \tau) \in \mathscr{P}_{\alpha}(M, S) \times \mathbb{R}^{+}$. Moreover if $(q, \tau) \in$ $\mathscr{P}_{\alpha}(M, S) \times \mathbb{R}^{+}$and $\left(q_{s}, \tau_{s}\right):=\Upsilon^{s}(q, \tau)$ then $\tau_{s}$ is bounded strictly away from zero as $s \rightarrow \infty$.

4. If $\alpha=0$ and $(q, \tau) \in \mathscr{P}_{0}(M, S) \times \mathbb{R}^{+}$satisfies $\omega_{+}(q, \tau)<\infty$, then if we define $\left(q_{s}, \tau_{s}\right):=\Upsilon^{s}(q, \tau)$ one has $\mathscr{S}_{L}\left(q_{s}, \tau_{s}\right) \rightarrow 0, \tau_{s} \rightarrow 0$ and $q_{s}$ converges to a constant path as $s \rightarrow \omega_{+}(q, \tau)$. If instead $\omega_{+}(q, \tau)=\infty$ then $\tau_{s}$ is strictly bounded away from zero as $s \rightarrow \infty$.

5. If $\alpha \neq 0$ then $\omega_{-}(q, \tau)=-\infty$ for all $(q, \tau) \in \mathscr{P}_{\alpha}(M, S) \times \mathbb{R}^{+}$.

6. Given $a>0$ define

$$
\mathscr{O}(a):=\left\{(q, \tau) \in \mathscr{P}_{0}(M, S) \times\left(0, k_{1} a\right) \mid \mathscr{S}_{L}(q, \tau)<a\right\},
$$

where $k_{1}>0$ was defined in (3.8). Then $\mathscr{O}(a) \cap \operatorname{Crit}\left(\mathscr{S}_{L}\right)=\emptyset$ for all $a>0$, and for any $a>0$, if $(q, \tau) \in \mathscr{O}(a)$ then $\Upsilon^{s}(q, \tau) \in \mathscr{O}(a)$ for all $s \in\left(\omega_{-}(q, \tau), 0\right]$. Finally if $(q, \tau) \in \mathscr{P}_{0}(M, S) \times \mathbb{R}^{+}$is such that $\omega_{-}(q, \tau)>-\infty$ and $\mathscr{S}_{L}(q, \tau) \geq$ a then there exists $s<0$ such that $\Upsilon^{s}(q, \tau) \in \mathscr{O}(a)$.

Given a refined pseudo-gradient $\mathbf{G}$ for $\mathscr{S}_{L}$ and a critical point $(q, \tau)$ of $\mathscr{S}_{L}$, we denote by $\mathbf{W}^{u}((q, \tau),-\mathbf{G})$ the extended unstable manifold, which by definition is the union of the unstable manifold $W^{u}((q, \tau),-\mathbf{G})$ together with the set of points one finds by following the forward orbit under $\Upsilon^{s}$ of elements $\left(q^{\prime}, \tau^{\prime}\right) \in W^{u}((q, \tau),-\mathbf{G})$ which do not converge in $\mathscr{P}(M, S) \times \mathbb{R}^{+}$as $s \rightarrow \omega_{+}\left(q^{\prime}, \tau^{\prime}\right)$. By Theorem 3.10.4 these are all of the form $(y, 0)$ for some point $y \in S$ (interpreted as usual as a constant path). These are the so-called critical points at infinity in the sense of Bahri [14]. In a similar vein it is convenient to define the following subset of $\mathscr{P}(M, S) \times[0, \infty)$ :

$$
\underline{\operatorname{Crit}}\left(\mathscr{S}_{L}\right):=\operatorname{Crit}\left(\mathscr{S}_{L}\right) \cup(S \times\{0\}) .
$$

Our non-degeneracy assumption implies that the functional $\mathscr{S}_{L}$ is actually Morse, but it is not "Morse at infinity", in the sense that the critical points at infinity (i.e. the set $S \times\{0\}$ ) form a Morse-Bott component of $\underline{\operatorname{Crit}}\left(\mathscr{S}_{L}\right)$. Thus we will need to work with flow lines with cascades to define the Morse (co)homology of $\mathscr{S}_{L}$, as we shall now explain. 


\subsection{The Morse complex}

To define the Morse complex we will need three pieces of auxiliary data. As with the construction of Rabinowitz Floer homology in Sect. 2.3, the construction is much simpler if $\alpha \neq 0$ (we can ignore the Morse function $\ell$ and there is no need for cascades). Nevertheless, for the sake of a uniform presentation, we do not treat this case separately.

- Firstly, let $\mathbf{G}$ denote a refined pseudo-gradient for $\mathscr{S}_{L}$, and as before write $\Upsilon^{s}$ for the local flow of $-\mathbf{G}$.

- Choose a Morse function $\ell: S \rightarrow \mathbb{R}$. In order to fit in with the approach taken in Sect. 2.3, it will be convenient to formally regard $\ell$ also as a function $\ell: \underline{\operatorname{Crit}}^{\alpha}\left(\mathscr{S}_{L}\right) \rightarrow \mathbb{R}$ by setting $\ell(q, \tau):=0$ for $(q, \tau) \in \mathrm{Crit}^{\alpha}\left(\mathscr{S}_{L}\right)$ and setting

$$
\ell(y, 0):=\ell(y) \text { for }(y, 0) \in S \times\{0\} .
$$

We denote by $C^{\alpha}(\ell) \subset \operatorname{Crit}^{\alpha}\left(\mathscr{S}_{L}\right)$ the set of critical points of $\ell$ (so that for $\alpha \neq 0$, $C^{\alpha}(\ell)=\operatorname{Crit}^{\alpha}\left(\mathscr{S}_{L}\right)$ and for $\left.\alpha=0, C^{0}(\ell)=\operatorname{Crit}^{0}\left(\mathscr{S}_{L}\right) \cup \operatorname{Crit}(\ell)\right)$, and given $-\infty \leq$ $a<b \leq \infty$ we define

$$
C^{\alpha}(\ell)_{a}^{b}:=C^{\alpha}(\ell) \cap{\underline{\mathrm{Crit}^{\alpha}}}^{\alpha}\left(\mathscr{S}_{L}\right)_{a}^{b},
$$

where by definition $\mathscr{S}_{L}(q, 0):=0$. It follows from Theorem 3.10 that if $b-a<\infty$ then $C^{\alpha}(\ell)_{a}^{b}$ is always finite.

- Thirdly, let $v$ denote a Riemannian metric on $S$ such that the flow $\psi^{t}$ of $-\nabla \ell=-\nabla_{\nu} \ell$ is Morse-Smale. As with $\ell$, we can formally regard $\psi^{t}$ as a flow on $\operatorname{Crit}^{\alpha}\left(\mathscr{S}_{L}\right)$ by defining $\psi^{t}(q, \tau):=(q, \tau)$ for all $(q, \tau) \in \operatorname{Crit}^{\alpha}\left(\mathscr{S}_{L}\right)$ and $t \in \mathbb{R}$.

Given $(q, \tau) \in C^{\alpha}(\ell)$, we denote by $i_{\ell}(q, \tau)$ the Morse index of $(q, \tau)$, seen as a critical point of $\ell$, so that $i_{\ell}(q, \tau):=\operatorname{dim} W^{u}((q, \tau) ;-\nabla \ell)$. Thus $i_{\ell}(q, \tau)=0$ unless $\tau=0$ and $q(t) \equiv y$ for some $y \in \operatorname{Crit}(\ell)$. Finally, let us define

$$
m_{\ell}(q, \tau):=m(q, \tau)+i_{\ell}(q, \tau)
$$

for $(q, \tau) \in C^{\alpha}(\ell)$, where by definition $m(y, 0):=0$. The Morse complex is defined with the aid of the spaces $\mathscr{W}\left(\left(q_{-}, \tau_{-}\right),\left(q_{+}, \tau_{+}\right) ; \ell\right)$ of gradient flow lines with cascades running between two critical points $\left(q_{-}, \tau_{-}\right)$and $\left(q_{+}, \tau_{+}\right)$of $C^{\alpha}(\ell)$. These spaces are defined entirely analogously to the spaces $\mathscr{M}\left(\left(x_{-}, \tau_{-}\right),\left(x_{+}, \tau_{+}\right)\right)$from Definition 2.21 , only we work with $\mathscr{S}_{L}$ and $\ell$ rather than $\mathscr{A}_{H}$ and $f$. We use the letter $\mathscr{W}$ instead of $\mathscr{M}$ to help distinguish between the two, and we include the " $\ell$ " in the notation because later on we will use these spaces with different choices of Morse function $\ell$. The next theorem, together with Theorem 3.12 below, follow from Theorem 3.10 exactly as in [10, Section 11]. The main ingredients are Abbondandolo and Majer's [6] infinite dimensional Morse theory and Frauenfelder's cascades approach to Morse-Bott homology (as described in [29] and Sect. 2.3).

Theorem 3.11 For a generic choice of $\mathbf{G}$ and $v$ the sets $\mathscr{W}\left(\left(q_{-}, \tau_{-}\right),\left(q_{+}, \tau_{+}\right) ; \ell\right)$ are all smooth manifolds of finite dimension

$$
\operatorname{dim} \mathscr{W}\left(\left(q_{-}, \tau_{-}\right),\left(q_{+}, \tau_{+}\right) ; \ell\right)=m_{\ell}\left(q_{-}, \tau_{-}\right)-m_{\ell}\left(q_{+}, \tau_{+}\right)-1 .
$$

Moreover if $m_{\ell}\left(q_{-}, \tau_{-}\right)-m_{\ell}\left(q_{+}, \tau_{+}\right)=1$ then $\mathscr{W}\left(\left(q_{-}, \tau_{-}\right),\left(q_{+}, \tau_{+}\right) ; \ell\right)$ is compact, and hence a finite set.

Denote by

$$
\mathrm{CM}_{*}^{\alpha}(L, \ell)_{a}^{b}:=C_{*}^{\alpha}(\ell)_{a}^{b} \otimes \mathbb{Z}_{2},
$$


where the grading $*$ is given by the function $m_{\ell}$. Given $\left(q_{ \pm}, \tau_{ \pm}\right) \in C^{\alpha}(\ell)_{a}^{b}$ with $m_{\ell}\left(q_{-}, \tau_{-}\right)=$ $m_{\ell}\left(q_{+}, \tau_{+}\right)+1$, we define the number $n_{\text {Morse }}\left(\left(q_{-}, \tau_{-}\right),\left(q_{+}, \tau_{+}\right) ; \ell\right) \in \mathbb{Z}_{2}$ to be the parity of the finite set $\mathscr{W}\left(\left(q_{-}, \tau_{-}\right),\left(q_{+}, \tau_{+}\right) ; \ell\right)$. If $m_{\ell}\left(q_{-}, \tau_{-}\right) \neq m_{\ell}\left(q_{+}, \tau_{+}\right)+1$, set $n_{\text {Morse }}\left(\left(q_{-}, \tau_{-}\right),\left(q_{+}, \tau_{+}\right) ; \ell\right)=0$. Now we define the boundary operator

$$
\partial_{a}^{b}=\partial_{a}^{b}(L, \mathbf{G}, \ell, v): \mathrm{CM}_{*}^{\alpha}(L, \ell)_{a}^{b} \rightarrow \mathrm{CM}_{*-1}^{\alpha}(L, \ell)_{a}^{b}
$$

as the linear extension of

$$
\left(q_{-}, \tau_{-}\right) \mapsto \sum_{\left(q_{+}, \tau_{+}\right) \in C^{\alpha}(\ell)_{a}^{b}} n_{\text {Morse }}\left(\left(q_{-}, \tau_{-}\right),\left(q_{+}, \tau_{+}\right) ; \ell\right)\left(q_{+}, \tau_{+}\right)
$$

The next result is the Morse homology theorem. Let us write

$$
\Lambda_{L}^{b}(\alpha):=\left\{(q, \tau) \in \mathscr{P}_{\alpha}(M, S) \times \mathbb{R}^{+} \mid \mathscr{S}_{L}(q, \tau)<b\right\} .
$$

Theorem 3.12 For a generic choice of $\mathbf{G}$ and $v$, it holds that $\partial_{a}^{b} \circ \partial_{a}^{b}=0$. Thus $\left\{\mathrm{CM}_{*}^{\alpha}(L, \ell), \partial_{a}^{b}\right\}$ forms a chain complex. The isomorphism class of this complex is independent of the choice of $\mathbf{G}, \ell$ and $v$. The associated homology, known as the Morse homology of $\mathscr{S}_{L}$, is isomorphic to the singular (co)homology of the pair $\left(\Lambda_{L}^{b}(\alpha), \Lambda_{L}^{a}(\alpha)\right)$.

$$
H M_{*}^{\alpha}(L)_{a}^{b} \cong H_{*}\left(\Lambda_{L}^{b}(\alpha), \Lambda_{L}^{a}(\alpha) ; \mathbb{Z}_{2}\right)
$$

In particular, if $b=\infty$ and $a<\inf \mathscr{S}_{L}$ then

$$
H M_{*}^{\alpha}(L):=H M_{*}^{\alpha}(L)_{a}^{\infty} \cong H_{*}\left(\mathscr{P}_{\alpha}(M, S) \times \mathbb{R}^{+} ; \mathbb{Z}_{2}\right) \cong H_{*}\left(P_{\alpha}(M, S) ; \mathbb{Z}_{2}\right) .
$$

One can also play the same game with cohomology. For reasons that will become clear in Sect. 3.11, it is convenient to use the Morse function $-\ell$ when defining the Morse cohomology. Given $-\infty \leq a<b \leq \infty$, let $C^{\alpha}(-\ell)_{a}^{b}$ denote the set of critical points $(q, \tau)$ of $-\ell$ with $a<\mathscr{S}_{L}(a, \tau)<b$. We grade $C^{\alpha}(-\ell)$ with $m_{-\ell}$. Note that $C^{\alpha}(\ell)_{a}^{b}=C^{\alpha}(-\ell)_{a}^{b}$ as sets but in general not as graded sets. Now set

$$
\mathrm{CM}_{\alpha}^{*}(L,-\ell)_{a}^{b}:=\prod_{(q, \tau) \in C_{*}^{\alpha}(-\ell)_{a}^{b}} \mathbb{Z}_{2}(q, \tau),
$$

and define

$$
\delta_{a}^{b}=\delta_{a}^{b}(L, \mathbf{G},-\ell, v): \mathrm{CM}_{\alpha}^{*}(L,-\ell)_{a}^{b} \rightarrow \mathrm{CM}_{\alpha}^{*+1}(L,-\ell)_{a}^{b}
$$

as the linear extension of

$$
\left(q_{+}, \tau_{+}\right) \mapsto \sum_{\left(q_{-}, \tau_{-}\right) \in C^{\alpha}(-\ell)_{a}^{b}} n_{\text {Morse }}\left(\left(q_{-}, \tau_{-}\right),\left(q_{+}, \tau_{+}\right) ;-\ell\right)\left(q_{-}, \tau_{-}\right)
$$

(here $n_{\text {Morse }}\left(\left(q_{-}, \tau_{-}\right),\left(q_{+}, \tau_{+}\right) ;-\ell\right)$ denotes the parity of the corresponding finite set

$$
\left.\mathscr{W}\left(\left(q_{-}, \tau_{-}\right),\left(q_{+}, \tau_{+}\right) ;-\ell\right)\right) .
$$

Then $\delta_{a}^{b} \circ \delta_{a}^{b}=0$, and hence $\left\{\mathrm{CM}_{\alpha}^{*}(L,-\ell)_{a}^{b}, \delta_{a}^{b}\right\}$ forms a cochain complex, whose cohomology computes the singular cohomology of the pair $\left(\Lambda_{L}^{b}(\alpha), \Lambda_{L}^{a}(\alpha)\right)$. 
3.7 Relating the two functionals $\mathscr{S}_{L}$ and $\mathscr{A}_{H}$

We will now study the relationship between the two functionals $\mathscr{S}_{L}$ and $\mathscr{A}_{H}$. The next lemma follows readily from the definitions.

Lemma 3.13 The following relationships between Crit $^{ \pm \alpha}\left(\mathscr{S}_{L}\right)$ and $\mathrm{Crit}^{\alpha}\left(\mathscr{A}_{H}\right)$ hold:

1. Given $(q, \tau) \in \operatorname{Crit}^{\alpha}\left(\mathscr{S}_{L}\right)$, define

$$
\begin{aligned}
& \psi_{+}(q, \tau):=(x, \tau) \text { where } x(t):=\left(q(t), \nabla L^{\mathrm{v}}(q(t), \dot{q}(t))\right) . \\
& \psi_{-}(q, \tau):=(\mathbb{I}(x),-\tau),
\end{aligned}
$$

where $\mathbb{I}(x)(t):=x(1-t)$. Then if $\alpha \neq 0$, one has

$$
\operatorname{Crit}^{\alpha}\left(\mathscr{A}_{H}\right)=\psi_{+}\left(\operatorname{Crit}^{\alpha}\left(\mathscr{S}_{L}\right)\right) \cup \psi_{-}\left(\operatorname{Crit}^{-\alpha}\left(S_{L}\right)\right)
$$

and moreover one has

$$
\mathscr{A}_{H}\left(\psi_{ \pm}(q, \tau)\right)= \pm \mathscr{S}_{L}(q, \tau) .
$$

2. Given any $(x, \tau) \in P\left(T^{*} M, N^{*} S\right) \times \mathbb{R}$ with $\tau>0$, if $q:=\pi \circ x$ then

$$
\begin{gathered}
\mathscr{A}_{H}(x, \tau) \leq \mathscr{S}_{L}(q, \tau), \\
\mathscr{A}_{H}(\mathbb{I}(x),-\tau) \geq-\mathscr{S}_{L}(q, \tau)
\end{gathered}
$$

with equality if and only if $x=\left(q, \nabla L^{\mathrm{v}}(q, \dot{q})\right)$.

3. Let $(q, \tau) \in P_{\alpha}(M, S) \times \mathbb{R}^{+}$denote a critical point of $\mathscr{S}_{L}$. Then for all $(\xi, h)$ it holds that

$$
d^{2} \mathscr{A}_{H}\left(\psi_{+}(q, \tau)\right)((\xi, h),(\xi, h)) \leq d^{2} \mathscr{S}_{L}(q, \tau)\left(\left(\xi^{\mathrm{h}}, h\right),\left(\xi^{\mathrm{h}}, h\right)\right),
$$

Let $(q, \tau) \in P_{-\alpha}(M, S) \times \mathbb{R}^{+}$denote a critical point of $\mathscr{S}_{L}$. Then for all $(\xi, h)$ it holds that

$$
d^{2} \mathscr{A}_{H}\left(\psi_{-}(q, \tau)\right)((\xi, h),(\xi, h)) \geq-d^{2} \mathscr{S}_{L}(q, \tau)\left(\left(\mathbb{I}(\xi)^{\mathrm{h}},-h\right),\left(\mathbb{I}(\xi)^{\mathrm{h}},-h\right)\right) .
$$

4. Given a critical point $(q, \tau)$, a pair $(\xi, h)$ lies in the kernel of the Hessian of $\mathscr{A}_{H}$ at $\psi_{+}(q, \tau)$ [resp. $\left.\psi_{-}(q, \tau)\right]$ if and only if the pair $\left(\xi^{\mathrm{h}}, h\right)\left[\right.$ resp. $\left.\left(\mathbb{I}(\xi)^{\mathrm{h}},-h\right)\right]$ lies in the kernel of the Hessian of $\mathscr{S}_{L}$ at $(q, \tau)$.

5. If $(q, \tau) \in \operatorname{Crit}\left(\mathscr{S}_{L}\right)$ then

$$
\chi(q, \tau)=\chi\left(\psi_{+}(q, \tau)\right)=-\chi\left(\psi_{-}(q, \tau)\right) .
$$

Next, we discuss the relations between the indices of the critical points. We first recall the following statement, which is an extension of the Morse index theorem of Duistermaat [28] to the twisted symplectic form $\omega$.

Theorem 3.14 Let $(q, \tau) \in \operatorname{Crit}^{\alpha}\left(\mathscr{S}_{L}\right)$. Then

$$
m_{\tau}(q)=\mu_{\mathrm{Ma}}\left(\psi_{-}(q, \tau)\right)+d-\frac{n}{2} .
$$

Proof We deduce this from the equivalent statement for the standard symplectic form $d \lambda_{\text {can }}$ (specifically, from [7, Corollary 4.2]) by arguing as follows: take a tubular neighborhood $W$ of $q([0,1])$ in $M$. Since $H^{2}(W)=0,\left.\sigma\right|_{W}=d \varphi$ for some $\varphi \in \Omega^{1}(W)$. The flow $\left.\phi_{H}^{t}\right|_{W}$ is conjugate to the flow $\psi_{H_{\varphi}}^{t}: T^{*} W \rightarrow T^{*} W$, where $H_{\varphi}(q, p)=H\left(q, p-\varphi_{q}\right)$ and $\psi_{H_{\varphi}}^{t}$ denotes the flow of the symplectic gradient of $H_{\varphi}$ with respect to the standard symplectic form $d \lambda_{\text {can }}$. Since both the Maslov index and the Morse index are local invariants, the theorem now follows directly from [7, Corollary 4.2]. 
Recall that in order to define the Lagrangian Rabinowitz Floer chain complex we need to pick a Morse function $f$ on $\mathrm{Crit}^{\alpha}\left(\mathscr{A}_{H}\right)$. It is convenient to choose $f$ and $\ell$ so that they satisfy the following properties.

1. For all $(q, \tau) \in \operatorname{Crit}^{\alpha}\left(\mathscr{S}_{L}\right)$ one has

$$
\ell(q, \tau)=f\left(\psi_{-}(q, \tau)\right)=f\left(\psi_{+}(q, \tau)\right) .
$$

2. The function $\ell$ has a unique minimum $y_{\min }$ and a unique maximum $y_{\max }$ for two points $y_{\min }, y_{\max } \in S$ and is self-indexing, that is, $\ell(y)=i_{\ell}(y)$ for all $y \in \operatorname{Crit}(\ell)$. Note that if $d=\operatorname{dim} S=0$ (i.e. $S=\{y\}$ and $N^{*} S=T_{y}^{*} M$ ) then we obviously have $y_{\min }=y_{\max }=y$, but that in all other cases clearly $y_{\min } \neq y_{\max }$.

3. For all $x \in \Sigma \cap N^{*} S$, we have $\ell(\pi(x)) \leq f(x, 0) \leq \ell(\pi(x))+1 / 2$.

4. Every critical point of $\left.f\right|_{\Sigma \cap N^{*} S \times\{0\}}$ lies above a critical point of $\ell$ and moreover for each critical point $y$ of $\ell$ there are exactly two critical points of $\left.f\right|_{\Sigma \cap N^{*} S \times\{0\}}$ in $\Sigma \cap T_{y}^{*} M \times\{0\}$. Denoting these two critical points by $\psi_{ \pm}(y, 0)$, it holds that

$$
\begin{aligned}
\ell(y) & =f\left(\psi_{-}(y, 0)\right)=f\left(\psi_{+}(y, 0)\right)-1 / 2, \\
i_{\ell}(y) & =i_{f}\left(\psi_{-}(y, 0)\right)=i_{f}\left(\psi_{+}(y, 0)\right)-n+d+1 .
\end{aligned}
$$

That such functions exist is explained in detail in [10, Appendix B]. With this choice of functions $f$ and $\ell$ the following relationships hold - the proof is an immediate application of Theorem 3.9, part (5) of Lemma 3.13, and Theorem 3.14.

Corollary 3.15 Let $(q, \tau) \in C^{\alpha}(\ell)$. Then

$$
m_{\ell}(q, \tau)= \begin{cases}\mu_{f}\left(\psi_{+}(q, \tau)\right), & \tau>0, \\ -\mu_{f}\left(\psi_{-}(q, \tau)\right)+2 d-n+1, & \tau>0, \\ \mu_{f}\left(\psi_{+}(q, \tau)\right), & \tau=0, \\ \mu_{f}\left(\psi_{-}(q, \tau)\right)-d+n-1, & \tau=0\end{cases}
$$

and

$$
m_{-\ell}(q, \tau)= \begin{cases}\mu_{f}\left(\psi_{+}(q, \tau)\right), & \tau>0, \\ -\mu_{f}\left(\psi_{-}(q, \tau)\right)+2 d-n+1, & \tau>0, \\ -\mu_{f}\left(\psi_{+}(q, \tau)\right)+d, & \tau=0, \\ -\mu_{f}\left(\psi_{-}(q, \tau)\right)+2 d-n+1, & \tau=0 .\end{cases}
$$

3.8 Computing the Lagrangian Rabinowitz Floer homology

In this section we state the key technical result of this paper, which is the extension of [10, Theorem 2] to our setting.

Theorem 3.16 (Computation of the Lagrangian Rabinowitz Floer homology) Let $f$ : $\operatorname{Crit}^{\alpha}\left(\mathscr{A}_{H}\right) \rightarrow \mathbb{R}$ and $\ell: S \rightarrow \mathbb{R}$ be Morse functions as specified above. Let $m$ and $v$ denote generically chosen Riemannian metrics on Crit $\left(\mathscr{A}_{H}\right)$ and $S$ respectively, such that the flows $\varphi^{t}$ and $\psi^{t}$ of $-\nabla f=-\nabla_{m} f$ and $-\nabla \ell=-\nabla_{\nu} \ell$ are Morse-Smale. Let $\mathbf{G}$ denote a generically chosen refined pseudo-gradient for $\mathscr{S}_{L}$, and let $\mathbf{J}=\left(J_{t}\right) \subset \mathscr{J}(X, \omega)$ denote a generic family of almost complex structures, such that $\sup _{t}\left\|J_{t}-J_{g}\right\|_{L^{\infty}}$ is sufficiently small.

Fix $-\infty<a<b<\infty$. Then there exists: 
1. An injective chain map

$$
\left(\Phi_{\mathrm{SA}}\right)_{a}^{b}: C M_{*}^{\alpha}(L, \ell)_{a}^{b} \rightarrow C R F_{*}^{\alpha}(H, f)_{a}^{b}
$$

which admits a left inverse $\left(\widehat{\Phi}_{\mathrm{SA}}\right)_{a}^{b}: C R F_{*}^{\alpha}(H, f)_{a}^{b} \rightarrow C M_{*}^{\alpha}(L, \ell)_{a}^{b}$.

2. A surjective chain map

$$
\left(\Phi_{\mathrm{AS}}\right)_{a}^{b}: C R F_{*}^{\alpha}(H, f)_{a}^{b} \rightarrow C M_{-\alpha}^{-*+2 d-n+1}(L,-\ell)_{-b}^{-a}
$$

which admits a right inverse $\left(\widehat{\Phi}_{\mathrm{AS}}\right)_{a}^{b}: C M_{-\alpha}^{-*+2 d-n+1}(L,-\ell)_{-b}^{-a} \rightarrow C R F_{*}^{\alpha}(H, f)_{b}^{a}$.

Moreover:

1. If $d<n / 2$ then $\Phi_{\mathrm{SA}}$ and $\Phi_{\mathrm{AS}}$ define chain complex isomorphisms

$$
\begin{aligned}
& \left(\Phi_{\mathrm{SA}}\right)_{a}^{b}: C M_{*}^{\alpha}(L, \ell)_{a}^{b} \cong C R F_{*}^{\alpha}(H, f)_{a}^{b}, \\
& \left(\Phi_{\mathrm{AS}}\right)_{a}^{b}: C R F_{*}^{\alpha}(H, f)_{a}^{b} \rightarrow C M_{-\alpha}^{-*+2 d-n+1}(L,-\ell)_{-b}^{-a},
\end{aligned}
$$

and thus in the limit $a \rightarrow-\infty, b \rightarrow \infty$, if we identify $H M_{*}^{\alpha}(L, \ell) \cong H_{*}\left(P_{\alpha}(M, S) ; \mathbb{Z}_{2}\right)$ and $H M_{-\alpha}^{*}(L,-\ell) \cong H^{*}\left(P_{-\alpha}(M, S) ; \mathbb{Z}_{2}\right)$ we deduce that

$$
\operatorname{RFH}_{*}^{\alpha}(H) \cong \begin{cases}H_{*}\left(P_{\alpha}(M, S) ; \mathbb{Z}_{2}\right), & * \geq 0, \\ 0, & 2 d-n+1<*<0, \\ H^{-*+2 d-n+1}\left(P_{-\alpha}(M, S) ; \mathbb{Z}_{2}\right), & * \leq 2 d-n+1 .\end{cases}
$$

2. If $\alpha \neq 0$ and $d \geq n / 2$, or if $\alpha=0$ and $d=n / 2$ and $n \geq 4$ then the composition $\left(\Phi_{\mathrm{AS}}\right)_{a}^{b} \circ\left(\Phi_{\mathrm{SA}}\right)_{a}^{b}: C M_{*}^{\alpha}(L, \ell)_{a}^{b} \rightarrow C M_{-\alpha}^{-*+2 d-n+1}(L,-\ell)_{-b}^{-a}$ is chain homotopic to zero, that is, there exists a homomorphism

$$
\Theta_{a}^{b}: C M_{*}^{\alpha}(L, \ell)_{a}^{b} \rightarrow C M_{-\alpha}^{-*+2 d-n}(L,-\ell)_{-b}^{-a}
$$

such that

$$
\left(\Phi_{\mathrm{AS}}\right)_{a}^{b} \circ\left(\Phi_{\mathrm{SA}}\right)_{a}^{b}=\Theta_{a}^{b} \circ \partial_{a}^{b}+\delta_{-b}^{-a} \circ \Theta_{a}^{b}
$$

Setting

$$
\Psi_{a}^{b}:=\left(\Phi_{\mathrm{SA}}\right)_{a}^{b}-\left(\widehat{\Phi}_{\mathrm{AS}}\right)_{a}^{b} \circ \Theta_{a}^{b} \circ \partial_{a}^{b}-\partial_{a}^{b} \circ\left(\widehat{\Phi}_{\mathrm{AS}}\right)_{a}^{b} \circ \Theta_{a}^{b},
$$

the map $\Psi_{a}^{b}$ is chain homotopic to $\left(\Phi_{\mathrm{SA}}\right)_{a}^{b}$, and satisfies $\left(\Phi_{\mathrm{AS}}\right)_{a}^{b} \circ \Psi_{a}^{b}=0$. Thus we obtain a short exact sequence of chain complexes

$$
0 \rightarrow C M_{*}^{\alpha}(L, \ell)_{a}^{b} \stackrel{\Psi_{a}^{b}}{\rightarrow} C R F_{*}^{\alpha}(H, f)_{a}^{b} \stackrel{\left(\Phi_{\mathrm{AS}}\right)_{a}^{b}}{\rightarrow} C M_{-\alpha}^{-*+2 d-n+1}(L,-\ell)_{-b}^{-a} \rightarrow 0
$$

Thus in the limit $a \rightarrow-\infty, b \rightarrow \infty$, if we identify $H M_{*}^{\alpha}(L, \ell) \cong H_{*}\left(P_{\alpha}(M, S) ; \mathbb{Z}_{2}\right)$ and $H M_{-\alpha}^{*}(L,-\ell) \cong H^{*}\left(P_{-\alpha}(M, S) ; \mathbb{Z}_{2}\right)$, then we obtain the long exact sequence

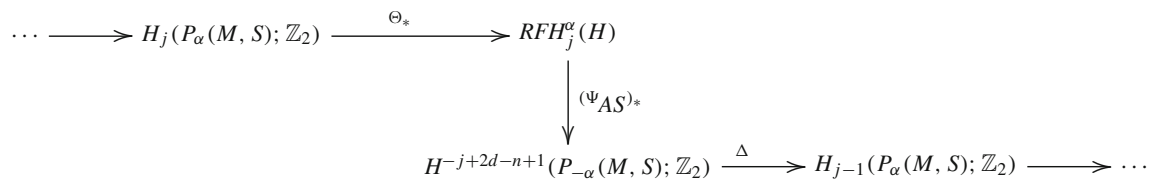


The connecting homomorphism $\Delta$ is identically zero unless $\alpha=0$ and $j=1$, in which case it is given by (recall by assumption when $\alpha=0$ one has $d=n / 2$ ):

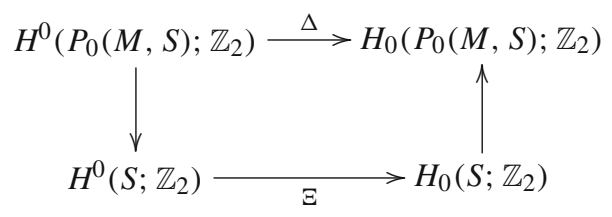

where

$$
\Xi(c):=P D\left(c \smile e\left(N^{*} S\right)\right),
$$

$\left(e\left(N^{*} S\right)\right.$ is the Euler class of $\left.N^{*} S \rightarrow S\right)$ and the vertical maps are the isomorphisms induced by the inclusion $S \hookrightarrow P_{0}(M, S)$.

The proof of this theorem is based on the corresponding result by Abbondandolo and Schwarz in [10], and its extension to twisted cotangent bundles in our earlier paper [37]. We will therefore omit many of the technical details in the exposition here, referring the reader to the beautiful and lucid exposition in [10], or the more detailed treatment given in [38]. Theorem 1.13 is an immediate consequence of this result.

3.9 The extended unstable manifolds with cascades $\mathbf{W}^{u}((q, \tau) ;-\mathbf{G},-\nabla \ell)$

We use the notation from Theorem 3.16. Recall the definition of the extended unstable manifold $\mathbf{W}^{u}((q, \tau)$; - G) introduced on page 30. We now introduce the extended unstable manifold with cascades, which we denote by $\mathbf{W}^{u}((q, \tau) ;-\mathbf{G},-\nabla \ell)$. Fix $(q, \tau) \in C^{\alpha}(\ell)$. Given $k \in \mathbb{N}$, let $\widetilde{\mathscr{W}}_{k}^{u}((q, \tau) ;-\nabla l)$ denote the set of tuples $(\mathbf{q}, \boldsymbol{\tau})=\left(q_{j}, \tau_{j}\right)_{1 \leq j \leq k}$ such that $\left(q_{j}, \tau_{j}\right) \in \mathscr{P}_{\alpha}(M, S) \times \mathbb{R}^{+}$for $j=1, \ldots, k-1$ and $\left(q_{k}, \tau_{k}\right)$ either belongs to $\mathscr{P}_{\alpha}(M, S) \times \mathbb{R}^{+}$ or is of the form $(y, 0)$ for some point $y \in S$. Moreover we insist that

$$
\begin{aligned}
& \left.\left(q_{1}, \tau_{1}\right) \in \mathbf{W}^{u}\left(W^{u}((q, \tau)) ;-\nabla \ell\right) ;-\mathbf{G}\right), \\
& \lim _{s \rightarrow-\infty} \Upsilon^{s}\left(q_{j+1}, \tau_{j+1}\right) \in \psi^{[0, \infty)}\left(\lim _{s \rightarrow \infty} \Upsilon^{s}\left(q_{j}, \tau_{j}\right)\right) \text { for } j=1, \ldots, k-1 .
\end{aligned}
$$

Of course, if $\alpha \neq 0$ then $\left(q_{k}, \tau_{k}\right)$ is always in $\mathscr{P}_{\alpha}(M, S) \times \mathbb{R}^{+}$. Let $\mathscr{W}_{k}^{u}((q, \tau) ;-\nabla l)$ denote the quotient of $\widetilde{\mathscr{W}}_{k}^{u}((q, \tau) ;-\nabla l)$ under the free $\mathbb{R}^{k-1}$ action given by

$$
\left(q_{j}, \tau_{j}\right)_{1 \leq j \leq k} \mapsto\left(\left(\Upsilon^{s_{j}}\left(q_{j}, \tau_{j}\right)\right)_{1 \leq j \leq k-1},\left(q_{k}, \tau_{k}\right)\right), \quad\left(s_{1}, \ldots, s_{k-1}\right) \in \mathbb{R}^{k-1} .
$$

Then set

$$
\mathbf{W}^{u}((q, \tau) ;-\mathbf{G},-\nabla \ell):=\bigcup_{k \in \mathbb{N}} \mathscr{W}_{k}^{u}((q, \tau) ;-\nabla l) .
$$

There is a well defined evaluation map

$$
\text { ev : } \mathbf{W}^{u}((q, \tau) ;-\mathbf{G},-\nabla \ell) \rightarrow\left(\mathscr{P}_{\alpha}(M, S) \times \mathbb{R}^{+}\right) \cup(S \times\{0\}),
$$

given by

$$
\operatorname{ev}(\mathbf{q}, \boldsymbol{\tau}):=\left(q_{k}, \tau_{k}\right) \text { for }(\mathbf{q}, \boldsymbol{\tau}) \in \mathscr{W}_{k}^{u}((q, \tau) ;-\nabla l) .
$$

For a generic choice of $\mathbf{G}$ and $v$ the spaces $\mathbf{W}^{u}((q, \tau) ;-\mathbf{G},-\nabla \ell)$ admit the structure of smooth manifolds of finite dimension 


$$
\operatorname{dim} \mathbf{W}^{u}((q, \tau) ;-\mathbf{G},-\nabla \ell)=m_{\ell}(q, \tau) .
$$

This can be proved using [29, Corollary A.16], and details can be found in [38, Section 12.1].

3.10 The chain map $\Phi_{S A}$

In this section we define a chain map

$$
\left(\Phi_{\mathrm{SA}}\right)_{a}^{b}: \mathrm{CM}_{*}^{\alpha}(L, \ell)_{a}^{b} \rightarrow \operatorname{CRF}_{*}^{\alpha}(H, h)_{a}^{b} .
$$

In order to define the chain map $\Phi_{\mathrm{SA}}$, one needs to construct a suitable moduli space. The first step is to define the space of positive half flow lines with cascades for $\mathscr{A}_{H}$, denoted by $\mathbf{M}^{s}(x, \eta)$. Here we write critical points of $\mathscr{A}_{H}$ as pairs $(x, \eta)$ rather than $(x, \tau)$, so as to minimize confusion below. Fix $(x, \eta) \in C^{\alpha}(f)$. Given $k \in \mathbb{N}$ let $\widetilde{\mathscr{M}_{k}^{s}}(x, \eta)$ denote the set of $k$-tuples of maps $\mathbf{u}=\left(u_{1}, \ldots, u_{k}\right)$ such that

$$
\begin{aligned}
& u_{1}:[0, \infty) \rightarrow P_{\alpha}\left(T^{*} M, N^{*} S\right) \times \mathbb{R} ; \\
& u_{2}, \ldots, u_{k}: \mathbb{R} \rightarrow P_{\alpha}\left(T^{*} M, N^{*} S\right) \times \mathbb{R},
\end{aligned}
$$

are all gradient flow lines of $(H, \mathbf{J})$ (that are possibly stationary solutions) and such that

$$
\begin{aligned}
& \lim _{s \rightarrow \infty} u_{k}(s) \in W^{s}((x, \eta) ;-\nabla f) ; \\
& \lim _{s \rightarrow-\infty} u_{j+1}(s) \in \varphi^{[0, \infty)}\left(\lim _{s \rightarrow \infty} u_{j}(s)\right) \text { for } j=1, \ldots, k-1 .
\end{aligned}
$$

Let $\mathscr{M}_{k}^{s}(x, \eta)$ denote the quotient of $\widetilde{\mathscr{M}_{k}^{s}}(x, \eta)$ under the free $\mathbb{R}^{k-1}$ action given by translation along the flow lines $u_{2}, \ldots, u_{k}$. Then put

$$
\mathbf{M}^{s}(x, \eta):=\bigcup_{k \in \mathbb{N}} \mathscr{M}_{k}^{s}(x, \eta)
$$

The space $\mathbf{M}^{s}(x, \eta)$ is not finite dimensional. However, by restricting where $u_{1}$ can "begin", we can cut it down to something finite dimensional. This is precisely what the moduli space $\mathscr{M}_{\mathrm{SA}}((q, \tau),(x, \eta))$ does. Fix $(q, \tau) \in C^{\alpha}(\ell)$ and define $\mathscr{M}_{\mathrm{SA}}((q, \tau),(x, \eta))$ to be the following subset of $\mathbf{W}^{u}((q, \tau) ;-\mathbf{G},-\nabla \ell) \times \mathbf{M}^{s}(x, \eta)$. Namely, an element

$$
(\mathbf{q}, \boldsymbol{\tau}, \mathbf{u}) \in \mathbf{W}^{u}((q, \tau) ;-\mathbf{G},-\nabla \ell) \times \mathbf{M}^{s}(x, \eta)
$$

with $(\mathbf{q}, \boldsymbol{\tau}) \in \mathscr{W}_{k}^{u}((q, \tau) ;-\nabla \ell)$ belongs to $\mathscr{M}_{\mathrm{SA}}((q, \tau),(x, \eta))$ if and only if, writing $u_{1}=$ $\left(x_{1}, \eta_{1}\right)$ one has

$$
\left(q_{k}, \tau_{k}\right)=\left(\pi \circ x_{1}(0, \cdot), \eta_{1}(0)\right) .
$$

This defines a Lagrangian boundary condition. This implies that we have a Fredholm problem, and since generically $\mathbf{W}^{u}((q, \tau) ;-\mathbf{G},-\nabla \ell)$ is a finite dimensional manifold, it follows that $\mathscr{M}_{\mathrm{SA}}((q, \tau),(x, \eta))$ can be seen as the zero set of a Fredholm operator. In fact, more is true.

Theorem 3.17 For a generic choice of $\mathbf{G}, \mathbf{J}, m$ and $v$, the spaces $\mathscr{M}_{\mathrm{SA}}((q, \tau),(x, \eta))$ are precompact smooth manifolds of finite dimension

$$
\operatorname{dim} \mathscr{M}_{\mathrm{SA}}((q, \tau),(x, \eta))=m_{\ell}(q, \tau)-\mu_{f}(x, \eta) .
$$

Proof The only complication with obtaining transversality is the presence of stationary solutions, which can appear if $(x, \eta)=\psi_{+}(q, \tau)$ or $(q, \tau)=(y, 0)$ for some $y \in S$ and $(x, \eta)=\psi_{ \pm}(y, 0)$. In the former case the first inequality of the third statement of Lemma 
3.13 forces the linearized operator defining the moduli space $\mathscr{M}_{\mathrm{SA}}\left((q, \tau), \psi_{+}(q, \tau)\right)$ to be an isomorphism (see [10, Lemma 6.2] or [9, Proposition 3.7]), and in the second two cases the four assumptions made earlier on the Morse functions $f$ and $\ell$ guarantee that the linearized operator defining the moduli spaces $\mathscr{M}_{\mathrm{SA}}\left((y, 0), \psi_{ \pm}(y, 0)\right)$ is surjective (see [10, Lemma 6.3]). The index computation can be proved by combining [12, Theorem 5.24] (a special case of this is given in [7, Proposition 7.3]) and the arguments from [20, Section 4]. Full details can be found in [38, Theorem 12.3]. Finally we address the precompactness statement. The key point here is the following chain of inequalities, which follow from part (2) of Lemma 3.13:

$$
\mathscr{S}_{L}(q, \tau) \geq \mathscr{S}_{L}\left(q_{k}, \tau_{k}\right)=\mathscr{S}_{L}\left(\pi \circ x_{1}(0, \cdot), \eta_{1}(0)\right) \geq \mathscr{A}_{H}\left(u_{1}(0, \cdot)\right) \geq \mathscr{A}_{H}(x, \eta) .
$$

More details can be found in [10, Section 6] and [38, Theorem 12.3].

Putting this together, we deduce that when $m_{\ell}(q, \tau)=\mu_{f}(x, \eta)$, the space $\mathscr{M}_{\mathrm{SA}}((q, \tau)$, $(x, \eta))$ is a finite set, and hence we can define $n_{\mathrm{SA}}((q, \tau),(x, \eta)) \in \mathbb{Z}_{2}$ to be its parity. If $m_{\ell}(q, \tau) \neq \mu_{f}(x, \eta)$, set $n_{\mathrm{SA}}((q, \tau),(x, \eta))=0$. Then define $\left(\Phi_{\mathrm{SA}}\right)_{a}^{b}: \mathrm{CM}_{*}^{\alpha}(L, \ell)_{a}^{b} \rightarrow$ $\mathrm{CRF}_{*}^{\alpha}(H, f)_{a}^{b}$ as the linear extension of

$$
(q, \tau) \mapsto \sum_{(x, \eta) \in C^{\alpha}(f)_{a}^{b}} n_{\mathrm{SA}}((q, \tau),(x, \eta))(x, \eta)
$$

(we are implicitly using (3.9) here to ensure that the choice of action window makes sense). A standard gluing argument shows that $\left(\Phi_{\mathrm{SA}}\right)_{a}^{b}$ is a chain map.

\subsection{The chain map $\Phi_{\text {AS }}$}

In this section we define a chain map

$$
\left(\Phi_{\mathrm{AS}}\right)_{a}^{b}: \mathrm{CRF}_{*}^{\alpha}(H, f)_{a}^{b} \rightarrow \mathrm{CM}_{-\alpha}^{-*+2 d-n+1}(L,-\ell)_{-b}^{-a} .
$$

It is defined in much the same way. One begins by defining a space $\mathbf{M}^{u}(x, \eta)$ of negative half flow lines with cascades. Given $k \in \mathbb{N}$ let $\widetilde{\mathscr{M}_{k}^{u}}(x, \eta)$ denote the denote the set of tuples of maps $\mathbf{u}=\left(u_{1}, \ldots, u_{k}\right)$ such that

$$
\begin{aligned}
& u_{1}, \ldots, u_{k-1}: \mathbb{R} \rightarrow P_{\alpha}\left(T^{*} M, N^{*} S\right) \times \mathbb{R} ; \\
& u_{k}:(-\infty, 0] \rightarrow P_{\alpha}\left(T^{*} M, N^{*} S\right) \times \mathbb{R},
\end{aligned}
$$

which are gradient flow lines of $(H, \mathbf{J})$ (that are possibly stationary solutions) and such that

$$
\lim _{s \rightarrow-\infty} u_{k}(s) \in W^{u}((x, \eta) ;-\nabla f),
$$

and such that

$$
\lim _{s \rightarrow-\infty} u_{j+1}(s) \in \varphi^{[0, \infty)}\left(\lim _{s \rightarrow \infty} u_{j}(s)\right) \text { for } j=1, \ldots, k-1 .
$$

Let $\mathscr{M}_{k}^{u}(x, \eta)$ denote the quotient of $\widetilde{\mathscr{M}_{k}^{u}}(x, \eta)$ under the free $\mathbb{R}^{k-1}$ action and put

$$
\mathbf{M}^{u}(x, \eta):=\bigcup_{k \in \mathbb{N}} \mathscr{M}_{k}^{u}(x, \eta)
$$

Now if $(x, \eta) \in C^{\alpha}(f)$ and $(q, \tau) \in C^{-\alpha}(-\ell)$, we define $\mathscr{M}_{\mathrm{AS}}((x, \eta),(q, \tau))$ to be the following subset of $\mathbf{W}^{u}((q, \tau) ;-\mathbf{G}, \nabla \ell) \times \mathbf{M}^{u}(x, \eta)$ (note here we are using the Morse function $-\ell$ ). Namely, an element

$$
(\boldsymbol{q}, \boldsymbol{\tau}, \mathbf{u}) \in \mathbf{W}^{u}((q, \tau) ;-\mathbf{G}, \nabla \ell) \times \mathbf{M}^{u}(x, \eta)
$$


with $(\mathbf{q}, \boldsymbol{\tau}) \in \mathscr{W}_{k}^{u}((q, \tau) ; \nabla l)$ and $\mathbf{u} \in \mathscr{M}_{p}^{u}(x, \eta)$ belongs to $\mathscr{M}_{\mathrm{AS}}((x, \eta),(q, \tau))$ if and only if, writing $u_{p}=\left(x_{p}, \eta_{p}\right)$ one has

$$
\left(q_{k}, \tau_{k}\right)=\left(\pi \circ x_{p}(0,-\cdot),-\eta_{p}(0)\right) .
$$

The following theorem is proved in the same way as Theorem 3.17. Details can be found in [10, Section 9] and [38, Section 12.3].

Theorem 3.18 For a generic choice of $\mathbf{G}, \mathbf{J}, m$ and $v$, the spaces $\mathscr{M}_{\mathrm{AS}}((x, \eta),(q, \tau))$ are precompact smooth manifolds of finite dimension

$$
\operatorname{dim} \mathscr{M}_{\mathrm{AS}}((x, \eta),(q, \tau))=\mu_{f}(x, \tau)+m_{-\ell}(q, \tau)+n-2 d-1 .
$$

We remark only that this time the key inequality responsible for compactness is the following: if $(\boldsymbol{q}, \boldsymbol{\tau}, \mathbf{u}) \in \mathscr{M}_{\mathrm{AS}}((x, \eta),(q, \tau))$ with $(\mathbf{q}, \boldsymbol{\tau}) \in \mathscr{W}_{k}^{u}((q, \tau) ; \nabla l)$ and $\mathbf{u} \in \mathscr{M}_{p}^{u}(x, \eta)$ then

$$
\mathscr{A}_{H}(x, \eta) \geq \mathscr{A}_{H}\left(u_{p}(0, \cdot)\right) \geq-\mathscr{S}_{L}\left(\pi \circ x_{p}(0,-\cdot),-\tau_{p}(0)\right) \geq-\mathscr{S}_{L}\left(q_{k}, \tau_{k}\right) \geq-\mathscr{S}_{L}(q, \tau) .
$$

Putting this together, we deduce that when $\mu_{f}(x, \eta)=-m_{-\ell}(q, \tau)+2 d-n+1$, the moduli space $\mathscr{M}_{\mathrm{AS}}((x, \eta),(q, \tau))$ is a finite set, and hence we can define $n_{\mathrm{AS}}((x, \eta),(q, \tau)) \in \mathbb{Z}_{2}$ to be its parity. If $\mu_{f}(x, \eta) \neq-m_{-\ell}(q, \tau)+1-n+2 d$ set $n_{\mathrm{AS}}((x, \eta),(q, \tau))=0$. Then define $\left(\Phi_{\mathrm{AS}}\right)_{a}^{b}: \mathrm{CRF}_{*}^{\alpha}(H, f)_{a}^{b} \rightarrow \mathrm{CM}_{-\alpha}^{-*+2 d-n+1}(L,-\ell)_{-b}^{-a}$ as the linear extension of

$$
(x, \eta) \mapsto \sum_{(q, \tau) \in C^{-\alpha}(\ell)_{-b}^{-a}} n_{\mathrm{AS}}((x, \eta),(q, \tau))(q, \tau)
$$

(this time we are implicitly using (3.10) here to ensure that the choice of action window makes sense). A standard gluing argument shows that $\left(\Phi_{\mathrm{AS}}\right)_{a}^{b}$ is a chain map.

\subsection{The chain homotopy $\Theta$}

We assume throughout this section that $d \geq n / 2$, and if $\alpha=0$ then we additionally assume $n \geq 4$ and that $d=n / 2$.

We will construct a chain homotopy

$$
\Theta_{a}^{b}: \mathrm{CM}_{*}^{\alpha}(L, \ell)_{a}^{b} \rightarrow \mathrm{CM}_{-\alpha}^{-*+2 d-n}(L,-\ell)_{-b}^{-a}
$$

which will have the property that

$$
\left(\Phi_{\mathrm{AS}}\right)_{a}^{b} \circ\left(\Phi_{\mathrm{SA}}\right)_{a}^{b}=\Theta_{a}^{b} \circ \partial_{a}^{b}+\delta_{-b}^{-a} \circ \Theta_{b}^{a} .
$$

This will involve counting a slightly different sort of object. Let $\mathscr{F}_{0}$ denote the set of pairs $(u, R)$ where $R \in \mathbb{R}^{+}$and $u=(x, \eta):[-R, R] \times[0,1] \rightarrow T^{*} M \times \mathbb{R}$ satisfies the Rabinowitz Floer equation. Given $k \geq 1$, let $\widetilde{\mathscr{F}}_{k}$ denote the set of tuples $(v, \mathbf{u}, w)$ where $\mathbf{u}=\left(u_{1}, \ldots, u_{k-1}\right)$ are gradient flow lines of $\mathscr{A}_{H}$ such that

$$
\lim _{s \rightarrow-\infty} u_{j+1}(s) \in \varphi^{[0, \infty)}\left(\lim _{s \rightarrow \infty} u_{j}(s)\right) \text { for } j=1, \ldots, k-2 .
$$

Next,

$$
\begin{aligned}
& v:[0, \infty) \rightarrow P_{\alpha}\left(T^{*} M, N^{*} S\right) \times \mathbb{R}, \\
& w:(-\infty, 0] \rightarrow P_{\alpha}\left(T^{*} M, N^{*} S\right) \times \mathbb{R}
\end{aligned}
$$


both satisfy the Rabinowitz Floer equation, with

$$
\lim _{s \rightarrow-\infty} u_{1}(s) \in \varphi^{[0, \infty)}\left(\lim _{s \rightarrow \infty} v(s)\right), \quad \lim _{s \rightarrow-\infty} w(s) \in \varphi^{[0, \infty)}\left(\lim _{s \rightarrow \infty} u_{k-1}(s)\right) .
$$

Let $\mathscr{F}_{k}$ denote the quotient of $\widetilde{\mathscr{F}}_{k}$ by dividing through by the $\mathbb{R}^{k-1}$ action on the curves $u_{1}, \ldots, u_{k-1}$. Put

$$
\mathscr{F}=\bigcup_{k \in \mathbb{N} \cup\{0\}} \mathscr{F}_{k} .
$$

Given $\left(q_{-}, \tau_{-}\right) \in C^{\alpha}(\ell)$ and $\left(q_{+}, \tau_{+}\right) \in C^{-\alpha}(-\ell)$, we define $\mathscr{M}_{\Theta}\left(\left(q_{-}, \tau_{-}\right),\left(q_{+}, \tau_{+}\right)\right)$to be the subset of points in

$$
\mathbf{W}^{u}\left(\left(q_{-}, \tau_{-}\right) ;-\mathbf{G},-\nabla \ell\right) \times \mathscr{F} \times \mathbf{W}^{u}\left(\left(q_{+}, \tau_{+}\right) ;-\mathbf{G}, \nabla \ell\right)
$$

satisfying:

1. If $\left((\mathbf{q}, \boldsymbol{\tau}),(u, R),\left(\mathbf{q}^{\prime}, \boldsymbol{\tau}^{\prime}\right)\right) \in \mathscr{M}_{\Theta}\left(\left(q_{-}, \tau_{-}\right),\left(q_{+}, \tau_{+}\right)\right)$with $(u, R) \in \mathscr{F}_{0},(\mathbf{q}, \boldsymbol{\tau}) \in$ $\mathscr{W}_{i}^{u}\left(\left(q_{-}, \tau_{-}\right) ;-\nabla l\right)$, and $\left(\mathbf{q}^{\prime}, \boldsymbol{\tau}^{\prime}\right) \in \mathscr{W}_{p}^{u}\left(\left(q_{+}, \tau_{+}\right) ; \nabla l\right)$, then writing $u=(x, \eta)$, we require that

$$
(\pi \circ x(-R, \cdot), \eta(-R))=\left(q_{i}, \tau_{i}\right), \quad(\pi \circ x(R,-\cdot),-\eta(R))=\left(q_{p}^{\prime}, \tau_{p}^{\prime}\right) .
$$

2. If $\left((\mathbf{q}, \boldsymbol{\tau}),(v, \mathbf{u}, w),\left(\mathbf{q}^{\prime}, \boldsymbol{\tau}^{\prime}\right)\right) \in \mathscr{M}_{\Theta}\left(\left(q_{-}, \tau_{-}\right),\left(q_{+}, \tau_{+}\right)\right)$with $(v, \mathbf{u}, w) \in \mathscr{F}_{k}$ for some $k \geq 1,(\mathbf{q}, \boldsymbol{\tau}) \in \mathscr{W}_{i}^{u}\left(\left(q_{-}, \tau_{-}\right) ;-\nabla l\right)$, and $\left(\mathbf{q}^{\prime}, \boldsymbol{\tau}^{\prime}\right) \in \mathscr{W}_{p}^{u}\left(\left(q_{+}, \tau_{+}\right) ; \nabla l\right)$, then writing $v=(x, \eta)$ and $w=\left(x^{\prime}, \eta^{\prime}\right)$, we require that

$$
(\pi \circ x(0, \cdot), \eta(0))=\left(q_{i}, \tau_{i}\right), \quad\left(\pi \circ x^{\prime}(0,-\cdot),-\eta^{\prime}(0)\right)=\left(q_{p}^{\prime}, \tau_{p}^{\prime}\right) .
$$

Let us note if $\left((\mathbf{q}, \boldsymbol{\tau}),(u, R),\left(\mathbf{q}^{\prime}, \boldsymbol{\tau}^{\prime}\right)\right) \in \mathscr{M}_{\Theta}\left(\left(q_{-}, \tau_{-}\right),\left(q_{+}, \tau_{+}\right)\right)$with $(u, R) \in \mathscr{F}_{0}$, $(\mathbf{q}, \boldsymbol{\tau}) \in \mathscr{W}_{i}^{u}\left(\left(q_{-}, \tau_{-}\right) ;-\nabla l\right)$, and $\left(\mathbf{q}^{\prime}, \boldsymbol{\tau}^{\prime}\right) \in \mathscr{W}_{p}^{u}\left(\left(q_{+}, \tau_{+}\right) ; \nabla l\right)$, then we have

$$
\begin{aligned}
\left.\mathscr{S}_{L}\left(q_{-}, \tau_{-}\right)\right) & \geq \mathscr{S}_{L}\left(q_{i}, \tau_{i}\right) \geq \mathscr{A}_{H}(u(-R, \cdot)) \geq \mathscr{A}_{H}(u(R, \cdot)) \\
& \geq-\mathscr{S}_{L}\left(q_{p}^{\prime}, \tau_{p}^{\prime}\right) \geq-\mathscr{S}_{L}\left(q_{+}, \tau_{+}\right) .
\end{aligned}
$$

Similarly if $\left((\mathbf{q}, \boldsymbol{\tau}),(v, \mathbf{u}, w),\left(\mathbf{q}^{\prime}, \boldsymbol{\tau}^{\prime}\right)\right) \in \mathscr{M}_{\Theta}\left(\left(q_{-}, \tau_{-}\right),\left(q_{+}, \tau_{+}\right)\right)$with $\mathbf{u} \in \mathscr{F}_{k}$ for some $k \geq 1,(\mathbf{q}, \boldsymbol{\tau}) \in \mathscr{W}_{i}^{u}\left(\left(q_{-}, \tau_{-}\right) ;-\nabla l\right)$, and $\left(\mathbf{q}^{\prime}, \boldsymbol{\tau}^{\prime}\right) \in \mathscr{W}_{p}^{u}\left(\left(q_{+}, \tau_{+}\right) ; \nabla l\right)$, then we have

$$
\mathscr{S}_{L}\left(q_{-}, \tau_{-}\right) \geq \mathscr{S}_{L}\left(q_{i}, \tau_{i}\right) \geq \mathscr{A}_{H}(v(0, \cdot)) \geq \mathscr{A}_{H}(w(0, \cdot)) \geq-\mathscr{S}_{L}\left(q_{p}^{\prime}, \tau_{p}^{\prime}\right) \geq-\mathscr{S}_{L}\left(q_{+}, \tau_{+}\right) .
$$

This time we have the following result. For more details we refer the reader to [10, Section 8] or [38, Section 12.4]. The latter reference explains exactly where the assumption that $d=n / 2$ with $n \geq 4$ if $\alpha=0$ is used.

Theorem 3.19 Denote by $C_{\Theta}^{\alpha}(\ell,-\ell) \subset C^{\alpha}(\ell) \times C^{-\alpha}(-\ell)$ the set of pairs $\left(q_{ \pm}, \tau_{ \pm}\right)$of critical points that satisfy

$$
m_{\ell}\left(q_{-}, \tau_{-}\right)+m_{-\ell}\left(q_{+}, \tau_{+}\right) \in\{2 d-n, 2 d-n+1\} .
$$

Then for a generic choice of $\mathbf{G}, \mathbf{J}, m$ and $\nu$, the spaces $\mathscr{M}_{\Theta}\left(\left(q_{-}, \tau_{-}\right),\left(q_{+}, \tau_{+}\right)\right)$for $\left(q_{ \pm}, \tau_{ \pm}\right) \in$ $C_{\Theta}^{\alpha}(\ell,-\ell)$ are precompact smooth manifolds of finite dimension

$$
\operatorname{dim} \mathscr{M}_{\Theta}\left(\left(q_{-}, \tau_{-}\right),\left(q_{+}, \tau_{+}\right)\right)=m_{\ell}\left(q_{-}, \tau_{-}\right)+m_{-\ell}\left(q_{+}, \tau_{+}\right)+n-2 d .
$$


Now we move onto the key proposition which implies Theorem 3.16. The first statement of Theorem 3.20 below shows that under our assumptions, if we are given $\left(q_{-}, \tau_{-}\right) \in C^{\alpha}(\ell)$ and $\left(q_{+}, \tau_{+}\right) \in C^{-\alpha}(-\ell)$ with $m_{\ell}\left(q_{-}, \tau_{-}\right)+m_{-\ell}\left(q_{+}, \tau_{+}\right)=2 d-n$ then we can define $n_{\Theta}\left(\left(q_{-}, \tau_{-}\right),\left(q_{+}, \tau_{+}\right)\right)$as the parity of the finite set $\mathscr{M}_{\Theta}\left(\left(q_{-}, \tau_{-}\right),\left(q_{+}, \tau_{+}\right)\right)$. This defines the chain map $\Theta_{a}^{b}$ (this time we are implicitly using (3.11) and (3.12) in order to ensure that the choice of action window makes sense). The fact that $\Theta_{a}^{b}$ is a chain homotopy between $\left(\Phi_{\mathrm{SA}}\right)_{a}^{b}$ and $\left(\Phi_{\mathrm{AS}}\right)_{a}^{b}$ involves studying the compactification of $\mathscr{M}_{\Theta}\left(\left(q_{-}, \tau_{-}\right),\left(q_{+}, \tau_{+}\right)\right)$by adding in the broken trajectories, and is the content of the second statement of the proposition below, which is taken from [10, Proposition 8.1]. Details of the proof in the Lagrangian case we study here can be found in [38, Section 12.10].

Proposition 3.20 Fix critical points $\left(q_{-}, \tau_{-}\right) \in C_{i}^{\alpha}(\ell)_{a}^{b}$ and $\left(q_{j}, \tau_{j}\right) \in C_{j}^{-\alpha}(-\ell)_{-b}^{-a}$. Recall we always assume $d \geq n / 2$ in this section, and if $\alpha=0$ then we require $d=n / 2$ and $n \geq 4$.

1. If $i+j=2 d-n$ then the moduli space $\mathscr{M}_{\Theta}\left(\left(q_{-}, \tau_{-}\right),\left(q_{+}, \tau_{+}\right)\right)$is compact.

2. If $i+j=2 d-n+1$ then the moduli space $\mathscr{M}_{\Theta}\left(\left(q_{-}, \tau_{-}\right),\left(q_{+}, \tau_{+}\right)\right)$is precompact, and we can identify the boundary $\partial \overline{\mathscr{M}}_{\Theta}\left(\left(q_{-}, \tau_{-}\right),\left(q_{+}, \tau_{+}\right)\right)$of the compactification $\overline{\mathscr{M}}_{\Theta}\left(\left(q_{-}, \tau_{-}\right),\left(q_{+}, \tau_{+}\right)\right)$as follows:

$$
\begin{aligned}
&=\left\{\overline{\mathscr{M}}_{\Theta}\left(\left(q_{-}, \tau_{-}\right),\left(q_{+}, \tau_{+}\right)\right)\right. \\
& \bigcup\left\{\bigcup_{(x, \eta) \in C_{i}^{\alpha}(f)_{a}^{b}} \mathscr{M}_{\mathrm{SA}}\left(\left(q_{-}, \tau_{-}\right),(x, \eta)\right) \times \mathscr{M}_{\mathrm{AS}}\left((x, \eta),\left(q_{+}, \tau_{+}\right)\right)\right\} \\
& \bigcup\left\{\bigcup_{(q, \tau) \in C_{i-1}^{\alpha}(\ell)_{b}^{a}} \mathscr{W}\left(\left(q_{-}, \tau_{-}\right),(q, \tau) ; \ell\right) \times \mathscr{M}_{\Theta}\left((q, \tau),\left(q_{+}, \tau_{+}\right)\right)\right\} \\
&\left.\bigcup_{\left(q^{\prime}, \tau^{\prime}\right) \in C_{j-1}^{\alpha}(-\ell)_{-b}^{-a}} \mathscr{M}_{\Theta}\left(\left(q_{-}, \tau_{-}\right),\left(q^{\prime}, \tau^{\prime}\right)\right) \times \mathscr{W}\left(\left(q_{+}, \tau_{+}\right),\left(q^{\prime}, \tau^{\prime}\right) ;-\ell\right)\right\} .
\end{aligned}
$$

Theorem 3.16 follows from this proposition; see [10, Section 9] or [38, Section 12] for the details.

Acknowledgments I would like to thank my Ph.D. adviser Gabriel P. Paternain for many helpful discussions. I am also extremely grateful to Alberto Abbondandolo, Peter Albers and Urs Frauenfelder, together with all the participants of the 2009-2010 Cambridge seminar on Rabinowitz Floer homology, for several stimulating remarks and insightful suggestions, and for pointing out errors in previous drafts of this work. This work forms part of my PhD thesis [38]. Finally, I am grateful to Irida Altman and the anonymous referees for their useful comments on making the paper more readable.

\section{References}

1. Abbas, C.: A note of V. I. Arnold's chord conjecture. Int. Math. Res. Not. 4, 217-222 (1999)

2. Albers, P., Frauenfelder, U.: Floer homology for negative line bundles and Reeb chords in prequantization spaces. J. Mod. Dyn. 33, 407-456 (2009)

3. Albers, P., Frauenfelder, U.: Leaf-wise intersections and Rabinowitz Floer homology. J. Topol. Anal. 21, 77-98 (2010)

4. Albers, P., Frauenfelder, U.: Infinitely many leaf-wise intersection points on cotangent bundles. In: Global Differential Geometry, Proceedings in Mathematics. Springer (2012)

5. Arnold, V.I., Givental, A.B.: Symplectic Geometry, Dynamical Systems, Encyclopedia of Mathematical Sciences, vol. 4. Springer, Berlin (1990) 
6. Abbondandolo, A., Majer, P.: Lectures on the Morse complex for infinite dimensional manifolds. In: Biran, P., Cornea, O., Lalonde, F. (eds.) Morse Theoretic Methods in Nonlinear Analysis and Symplectic Topology, Nato Science Series II: Mathematics, Physics and Chemistry, vol. 217, pp. 1-74. Springer (2006)

7. Abbondandolo, A., Portaluri, A., Schwarz, M.: The homology of path spaces and Floer homology with conormal boundary conditions. J. Fixed Point Theory Appl. 4(2), 263-293 (2008)

8. Arnold, V.I.: First steps in symplectic topology. Russ. Math. Surv. 41, 1-21 (1986)

9. Abbondandolo, A., Schwarz, M.: On the Floer homology of cotangent bundles. Commun. Pure Appl. Math. 59, 254-316 (2006)

10. Abbondandolo, A., Schwarz, M.: Estimates and computations in Rabinowitz-Floer homology. J. Topol. Anal. 1(4), 307-405 (2009)

11. Abbondandolo, A., Schwarz, M.: A smooth pseudo-gradient for the Lagrangian action functional. Adv. Nonlinear Stud. 9, 597-623 (2009)

12. Abbondandolo, A., Schwarz, M.: Floer homology of cotangent bundles and the loop product. Geom. Topol. 14, 1569-1722 (2010)

13. Abouzaid, M., Seidel, P.: An open string analogue of Viterbo functoriality. Geom. Topol. 14, 627-718 (2010)

14. Bahri, A.: Critical Points at Infinity in Some Variational Problems, Pitman Research Notes in Mathematics, vol. 182. Longman, London (1989)

15. Bourgeois, F., Ekholm, T., Eliashberg, Y.: Effect of Legendrian surgery. arXiv:0911.0026 (2009)

16. Bourgeois, F., Oancea, A.: Symplectic homology, autonomous Hamiltonians, and Morse-Bott moduli spaces. Duke Math. J. 146(1), 71-174 (2009)

17. Bounya, C.: An exact triangle for the wrapped Floer homology of the Lagrangian Rabinowitz functional, In preparation

18. Bourgeois, F.: A Morse-Bott approach to contact homology. In: Eliashberg, Y., Khesin, B., Lalonde, F. (eds.) Symplectic and Contact Topology: Interactions and Perspectives, Fields Institute Communications, vol. 35. American Mathematical Society, USA (2003)

19. Burns, K., Paternain, G.P.: Anosov magnetic flows, critical values and topological entropy. Nonlinearity 15, 281-314 (2002)

20. Cieliebak, K., Frauenfelder, U.: A Floer homology for exact contact embeddings. Pac. J. Math. 239(2), 216-251 (2009)

21. Cieliebak, K., Frauenfelder, U., Oancea, A.: Rabinowitz Floer homology and symplectic homology. Ann. Inst. Fourier 43(6), 957-1015 (2010)

22. Cieliebak, K., Frauenfelder, U., Paternain, G.P.: Symplectic topology of Mañé's critical values. Geom. Topol. 14, 1765-1870 (2010)

23. Cieliebak, K., Ginzburg, V., Kerman, E.: Symplectic homology and periodic orbits near symplectic submanifolds. Comment Math. Helv. 74, 554-581 (2004)

24. Contreras, G., Iturriaga, R.: Global Minimizers of Autonomous Lagrangians, Colloqio Brasileiro de Matematica, vol. 22. IMPA, Rio de Janeiro (1999)

25. Cieliebak, K.: Handle attaching in symplectic homology and the chord conjecture. J. Eur. Math. Soc. 4(2), 115-142 (2002)

26. Contreras, G., Iturriaga, R., Paternain, G.P., Paternain, M.: The Palais-Smale condition and Mañé's critical values. Ann. Henri Poincaré 1(4), 655-684 (2000)

27. Contreras, G.: The Palais-Smale condition on contact type energy levels for convex Lagrangian systems. Calc. Var. Partial Differ. Eqs. 27(3), 321-395 (2006)

28. Duistermaat, J.J.: On the Morse index in variational calculus. Adv. Math. 21, 173-195 (1976)

29. Frauenfelder, U.: The Arnold-Givental conjecture and moment Floer homology. Int. Math. Res. Not. 42, 2179-2269 (2004)

30. Ginzburg, V.: On closed trajectories of a charge in a magnetic field. In: Thomas, C.B. (ed.) An Application of Symplectic Geometry, Contact and Symplectic Geometry (Cambridge, 1994), Publications of the Newton Institute, vol. 8, pp. 131-148. Cambridge University Press, Cambridge (1996)

31. Givental, A.: Nonlinear generalization of the Maslov index. Adv. Sov. Math. 1, 71-103 (1990)

32. Givental, A.: The nonlinear Maslov index, Geometry of Low-Dimensional manifolds. In: Donaldson, S. (ed.) LMS Lecture Note Series, vol. 151, pp. 35-43. Cambridge University Press, Cambridge (1990)

33. Hutchings, M., Taubes, C.: Proof of the Arnold chord conjecture in three dimensions I. Math. Res. Lett. 18(2), 295-313 (2011)

34. Hutchings, M., Taubes, C.: Proof of the rnold chord conjecture in three dimensions II. arXiv:1111.3324 (2011)

35. Hofer, H., Wysocki, K., Zehnder, E.: The dynamics on three-dimensional strictly convex energy surfaces. Ann. Math. 148(1), 197-289 (1998) 
36. Mañé, R.: Lagrangian Flows: The Dynamics of Globally Minimizing Orbits, Pitman Research Notes in Math., vol. 362, pp. 120-131. Longman, London (1996)

37. Merry, W.J.: On the Rabinowitz Floer homology of twisted cotangent bundles. Calc. Var. Partial Differ. Eqs. 42(3-4), 355-404 (2011)

38. Merry, W. J.: Rabinowitz Floer homology and Mañé supercritical hypersurfaces, Ph.D. thesis. University of Cambridge. Available online at http://www.math.ethz.ch/merrywi (2011)

39. Macarini, L., Merry, W.J., Paternain, G.P.: On the growth rate of leaf-wise intersections. J. Symplectic Geom. 10, 601-653 (2012)

40. Mohnke, K.: Holomorphic disks and the Chord conjecture. Ann. Math. 154, 219-222 (2001)

41. Moser, J.: A fixed point theorem in symplectic geometry. Acta. Math. 141(1-2), 17-34 (1978)

42. Merry, W.J., Paternain, G.P.: Index computations in Rabinowitz Floer homology. J. Fixed Point Theory Appl. 10(1), 88-111 (2011)

43. McDuff, D., Salamon, D.: J-holomorphic Curves and Symplectic Topology, vol. 52. Colloquim Publications, American Mathematical Society, Providence (2012)

44. Paternain, G.P.: Magnetic rigidity of horocycle flows. Pac. J. Math. 225, 301-323 (2006)

45. Pozniak, M.: Floer homology, Novikov rings and clean intersections. In: Eliashberg, Y., Fuchs, D., Ratiu, T., Weinstein, A. (eds.) Northern California Symplectic Geometry Seminar, vol. 196, 2nd edn, pp. 119181. American Mathematical Society, Providence (1999)

46. Ritter, A.F.: Topological quantum field theory structure on symplectic cohomology. J. Topol. 6(2), 391489 (2013)

47. Robbin, J., Salamon, D.: The Maslov index for paths. Topology 32, 827-844 (1993)

48. Salamon, D.: Lectures on Floer homology. In: Eliashberg, Y., Traynor, L. (eds.) Symplectic Geometry and Topology, IAS/Park City Math Series, vol. 7, pp. 143-225. American Mathematical Society, Providence (1999)

49. Schwarz, M.: On the action spectrum for closed symplectically aspherical manifolds. Pac. J. Math. 193(2), 419-461 (2000) 